\title{
Palladium-Catalyzed Decarbonylative Cross-Coupling of Azinecarboxylates with Arylboronic Acids
}

\author{
Kei Muto ${ }^{1}$, Taito Hatakeyama ${ }^{2}$, Kenichiro Itami $^{3,4}$, and Junichiro Yamaguchi ${ }^{1 *}$ \\ ${ }^{1}$ Department of Applied Chemistry, Waseda University, 3-4-1 Ohkubo, Shinjuku, Tokyo 169-8555, Japan \\ ${ }^{2}$ Central Research Laboratory Technology and Development Division, Kanto Chemicals Co. Inc., Saitama \\ 340-0003, Japan \\ 3 Institute of Transformative Bio-Molecules (WPI-ITbM) and Graduate School of Science, Nagoya \\ University, Chikusa, Nagoya 464-8602, Japan \\ 4 JST, ERATO, Itami Molecular Nanocarbon Project, Nagoya University, Chikusa, Nagoya 464-8602, \\ Japan \\ E-mail: itami@chem.nagoya-u.ac.jp,junyamaguchi@waseda.jp
}

\section{Table of Contents}

1. General

2. Preparation of Starting Materials

3. Pd-Catalyzed Decarbonylative Cross-Coupling

4. Condition Screening

5. ${ }^{1} \mathrm{H}$ NMR and ${ }^{13} \mathrm{C}$ NMR Spectra
$\mathrm{S} 2$

S3-S5

S6-S13

S14

S15-S66 


\section{General}

Unless otherwise noted, all materials including dry solvents were obtained from commercial suppliers and used as received. $\mathrm{Pd}(\mathrm{OAc})_{2}$ and $\mathrm{Na}_{2} \mathrm{CO}_{3}$ were obtained from Kanto Chemicals. 1,2-Bis(dicyclohexylphosphino)ethane (dcype) was obtained from Kanto Chemical. Phenyl picolinate (1A) 1, phenyl pyrazine-2-carboxylate (1D) $)^{1}, \quad$ phenyl nicotinate $(\mathbf{1 I})^{1}$, 3,4-bis(dicyclohexylphosphino)thiophene (dcypt) ${ }^{2}$ were synthesized according to procedures reported in the literature. Unless otherwise noted, all coupling reactions were performed with dry solvents under an atmosphere of argon in dried glassware using standard vacuum-line techniques. All work-up and purification procedures were carried out with reagent-grade solvents in air.

Analytical thin-layer chromatography (TLC) was performed using E. Merck silica gel $60 \mathrm{~F}_{254}$ precoated plates $(0.25 \mathrm{~mm})$. The developed chromatogram was analyzed by UV lamp (254 nm). Flash column chromatography was performed with E. Merck silica gel 60 (230-400 mesh). High-resolution mass spectra (HRMS) were obtained from a JMS-T100TD instrument (DART) and Thermo Scientific Exactive Plus Orbitrap MS (ESI). Nuclear magnetic resonance (NMR) spectra were recorded on a JEOL JNM-ECA-400 $\left({ }^{1} \mathrm{H} 400 \mathrm{MHz},{ }^{13} \mathrm{C} 100 \mathrm{MHz}\right)$ and JEOL JNM-ECA-600 $\left({ }^{1} \mathrm{H} 600 \mathrm{MHz},{ }^{13} \mathrm{C} 150\right.$ $\mathrm{MHz}$ ) spectrometer. Chemical shifts for ${ }^{1} \mathrm{H}$ NMR are expressed in parts per million (ppm) relative to tetramethylsilane $(\delta 0.00 \mathrm{ppm})$. Chemical shifts for ${ }^{13} \mathrm{C}$ NMR are expressed in ppm relative to $\mathrm{CDCl}_{3}$ $(\delta 77.0 \mathrm{ppm})$. Data are reported as follows: chemical shift, multiplicity $(\mathrm{s}=$ singlet, $\mathrm{d}=$ doublet, $\mathrm{dd}=$ doublet of doublets, $\mathrm{t}=$ triplet, $\mathrm{q}=$ quartet, $\mathrm{m}=$ multiplet, $\mathrm{br}=$ broad signal $)$, coupling constant $(\mathrm{Hz})$, and integration.

1. Amaike, K.; Muto, K.; Yamaguchi, J.; Itami, K. J. Am. Chem. Soc. 2012, 134, 13573.

2. Takise, R.; Muto, K.; Yamaguchi, J.; Itami, K. Angew. Chem., Int. Ed., 2014, 53, 6791. 


\section{Preparation of Starting Materials}

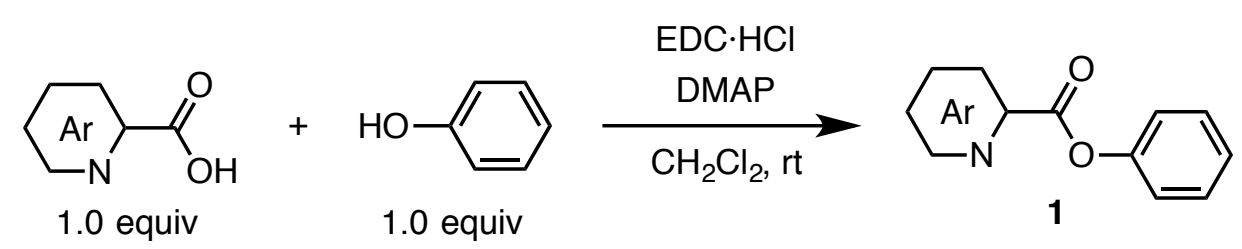

To a round-bottomed flask containing a magnetic stirring bar and carboxylic acid (1.0 equiv) were added phenol (1.0 equiv), 1-(3-dimethylaminopropyl)-3-ethylcarbodiimide hydrochloride (EDC·HCl: 1.2 equiv), $\mathrm{N}, \mathrm{N}$-dimethyl-4-aminopyridine (DMAP: 0.25 equiv) and $\mathrm{CH}_{2} \mathrm{Cl}_{2}(0.50 \mathrm{M})$. After stirring the mixture for several hours with monitoring reaction progress with TLC, the reaction was quenched with saturated $\mathrm{NaHCO}_{3}$ aq and extracted three times with $\mathrm{CH}_{2} \mathrm{Cl}_{2}$. The combined organic layer was dried over $\mathrm{Na}_{2} \mathrm{SO}_{4}$, filtrated, and concentrated in vacuo. The residue was purified by flash column chromatography to afford the corresponding phenyl ester $\mathbf{1}$.

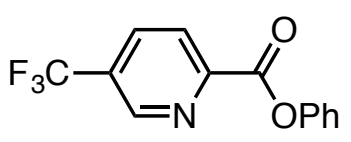

1B

Phenyl 5-(trifluoromethyl)picolinate (1B): Purification by flash column chromatography (hexane/EtOAc $=5: 1$ to 2:1) afforded $\mathbf{1 B}$ as a white solid $\left(524.0 \mathrm{mg}, 3.0 \mathrm{mmol} \mathrm{scale}, 65 \%\right.$ yield). ${ }^{1} \mathrm{H}$ NMR $\left(600 \mathrm{MHz}, \mathrm{CDCl}_{3}\right) \delta 9.10(\mathrm{~s}, 1 \mathrm{H}), 8.41(\mathrm{~d}, J=8.4 \mathrm{~Hz}, 1 \mathrm{H}), 8.18(\mathrm{~d}, J=8.4 \mathrm{~Hz}, 1 \mathrm{H}), 7.48-7.45$ $(\mathrm{m}, 2 \mathrm{H}), 7.32(\mathrm{t}, J=7.8 \mathrm{~Hz}, 1 \mathrm{H}), 7.29-7.26(\mathrm{~m}, 2 \mathrm{H}) ;{ }^{13} \mathrm{C} \mathrm{NMR}\left(100 \mathrm{MHz}, \mathrm{CDCl}_{3}\right) \delta 162.6,150.6$, $150.5,146.9\left(\mathrm{q}, J_{\mathrm{C}-\mathrm{F}}=3.8 \mathrm{~Hz}\right), 134.6\left(\mathrm{q}, J_{\mathrm{C}-\mathrm{F}}=2.8 \mathrm{~Hz}\right), 129.7\left(\mathrm{q}, J_{\mathrm{C}-\mathrm{F}}=34.4 \mathrm{~Hz}\right), 129.6,126.4,125.4$, $122.8\left(\mathrm{q}, J_{\mathrm{C}-\mathrm{F}}=278 \mathrm{~Hz}\right), 121.4$; HRMS (ESI) $\mathrm{m} / \mathrm{z}$ calcd for $\mathrm{C}_{13} \mathrm{H}_{9} \mathrm{~F}_{3} \mathrm{NO}_{2}{ }^{+}[\mathrm{M}+\mathrm{H}]^{+}: 268.0580$ found 268.0577.

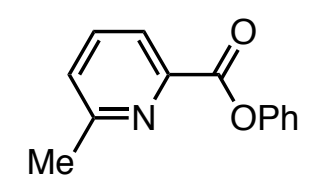

1C

Phenyl 6-methylpicolinate (1C): Purification by flash column chromatography (hexane/EtOAc $=5: 1$ to $2: 1)$ afforded $1 \mathrm{C}$ as a white solid $\left(662.0 \mathrm{mg}, 5.0 \mathrm{mmol}\right.$ scale, $62 \%$ yield). ${ }^{1} \mathrm{H}$ NMR (600 MHz, $\left.\mathrm{CDCl}_{3}\right) \delta 8.10(\mathrm{~d}, J=8.4 \mathrm{~Hz}, 1 \mathrm{H}), 7.79(\mathrm{t}, J=7.2 \mathrm{~Hz}, 1 \mathrm{H}), 7.45-7.39(\mathrm{~m}, 3 \mathrm{H}), 7.30-7.22(\mathrm{~m}, 3 \mathrm{H})$, 2.72 (s, 3H); ${ }^{13} \mathrm{C}$ NMR (100 MHz, $\left.\mathrm{CDCl}_{3}\right) \delta 163.9,159.2,150.9,146.8,137.1,129.3,127.2,125.9$, 122.9, 121.6, 24.6; HRMS (ESI) $\mathrm{m} / z$ calcd for $\mathrm{C}_{13} \mathrm{H}_{11} \mathrm{NO}_{2} \mathrm{Na}^{+}[\mathrm{M}+\mathrm{Na}]^{+}: 236.0682$ found 236.0680.<smiles>Cc1cnc(C(=O)Oc2ccccc2)cn1</smiles> 
Phenyl 5-methylpyrazine-2-carboxylate (1E): Purification by flash column chromatography (hexane/EtOAc $=5: 1$ to 1:1) afforded $\mathbf{1 E}$ as a white solid (492 $\mathrm{mg}, 5 \mathrm{mmol} \mathrm{scale}, 46 \%$ yield). ${ }^{1} \mathrm{H}$ NMR (400 MHz, $\left.\mathrm{CDCl}_{3}\right) \delta 9.34(\mathrm{~s}, 1 \mathrm{H}), 8.67(\mathrm{~s}, 1 \mathrm{H}), 7.46(\mathrm{t}, J=7.6 \mathrm{~Hz}, 2 \mathrm{H}), 7.31(\mathrm{t}, J=7.6 \mathrm{~Hz}, 1 \mathrm{H})$, $7.26(\mathrm{~d}, J=7.6 \mathrm{~Hz}, 2 \mathrm{H}), 2.72(\mathrm{~s}, 3 \mathrm{H}) ;{ }^{13} \mathrm{C} \mathrm{NMR}\left(100 \mathrm{MHz}, \mathrm{CDCl}_{3}\right) \delta 162.7,158.3,150.4,145.8$, 144.4, 140.0, 129.5, 126.3, 121.4, 22.0; HRMS (ESI) $m / z$ calcd for $\mathrm{C}_{12} \mathrm{H}_{10} \mathrm{~N}_{2} \mathrm{O}_{2} \mathrm{Na}^{+}[\mathrm{M}+\mathrm{Na}]^{+}: 237.0634$ found 237.0632 .

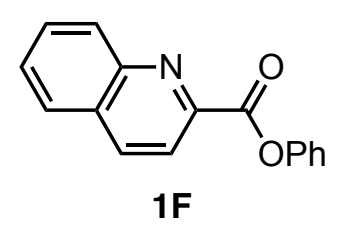

Phenyl quinoline-2-carboxylate (1F): Purification by flash column chromatography (hexane/EtOAc $=10: 1$ to $3: 1)$ afforded $\mathbf{1 F}$ as a white solid (1.83 g, $10 \mathrm{mmol}$ scale, $73 \%$ yield). ${ }^{1} \mathrm{H}$ NMR (400 MHz, $\left.\mathrm{CDCl}_{3}\right) \delta 8.38(\mathrm{~d}, J=8.8 \mathrm{~Hz}, 2 \mathrm{H}), 8.33(\mathrm{~d}, J=8.8 \mathrm{~Hz}, 1 \mathrm{H}), 7.93(\mathrm{~d}, J=8.4 \mathrm{~Hz}, 1 \mathrm{H}), 7.84(\mathrm{ddd}, J=$ $8.8,8.4,1.6 \mathrm{~Hz}, 1 \mathrm{H}), 7.70(\mathrm{td}, J=8.4,1.6 \mathrm{~Hz}, 1 \mathrm{H}), 7.52-7.42(\mathrm{~m}, 2 \mathrm{H}), 7.36-7.28(\mathrm{~m}, 3 \mathrm{H}) ;{ }^{13} \mathrm{C} \mathrm{NMR}$ $\left(100 \mathrm{MHz}, \mathrm{CDCl}_{3}\right) \delta$ 164.1, 151.0, 147.7, 147.4, 137.4, 130.8, 130.4, 129.5, 128.9, 127.6, 126.1, 121.8, 121.4 (one peak is missing because of overlap); HRMS (ESI) $\mathrm{m} / z$ calcd for $\mathrm{C}_{16} \mathrm{H}_{11} \mathrm{NO}_{2} \mathrm{Na}^{+}[\mathrm{M}+\mathrm{Na}]^{+}$: 272.0682 found 272.0678 .

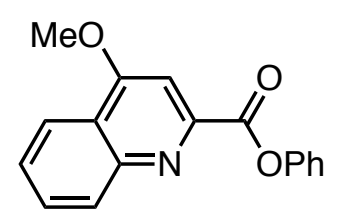

$1 G$

Phenyl 4-methoxyquinoline-2-carboxylate (1G): Purification by flash column chromatography (hexane/EtOAc $=5: 1$ to 3:1) afforded $\mathbf{1 G}$ as a white solid $\left(769.8 \mathrm{mg}, 5.0 \mathrm{mmol}\right.$ scale, $55 \%$ yield). ${ }^{1} \mathrm{H}$ NMR (400 MHz, $\left.\mathrm{CDCl}_{3}\right) \delta 8.28(\mathrm{t}, J=8.4 \mathrm{~Hz}, 2 \mathrm{H}), 7.80(\mathrm{ddd}, J=8.4,6.8,1.6 \mathrm{~Hz}, 1 \mathrm{H}), 7.69(\mathrm{~s}, 1 \mathrm{H})$, $7.64(\mathrm{dd}, J=8.4,7.6 \mathrm{~Hz}, 1 \mathrm{H}), 7.49-7.44(\mathrm{~m}, 2 \mathrm{H}), 7.33-7.28(\mathrm{~m}, 3 \mathrm{H}), 4.16(\mathrm{~s}, 3 \mathrm{H}) ;{ }^{13} \mathrm{C}$ NMR $(100$ $\left.\mathrm{MHz}, \mathrm{CDCl}_{3}\right) \delta 164.6,163.4,151.2,148.6,148.5,130.6,130.4,129.5,127.9,126.1,122.3,121.85$, 121.79, 100.5, 56.1; HRMS (ESI) $\mathrm{m} / z$ calcd for $\mathrm{C}_{17} \mathrm{H}_{13} \mathrm{NO}_{3} \mathrm{Na}^{+}[\mathrm{M}+\mathrm{Na}]^{+}: 302.0788$ found 302.0788 .

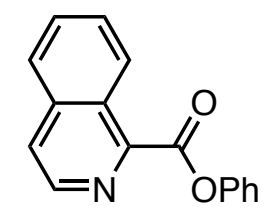

$1 \mathrm{H}$

Phenyl isoquinoline-1-carboxylate $\mathbf{( 1 H ) :}$ Purification by flash column chromatography (hexane/EtOAc $=5: 1$ to $3: 1)$ afforded $\mathbf{1 H}$ as a white solid $\left(1.63 \mathrm{~g}, 10 \mathrm{mmol}\right.$ scale, $65 \%$ yield). ${ }^{1} \mathrm{H}$ 
NMR (400 MHz, $\left.\mathrm{CDCl}_{3}\right) \delta 8.93(\mathrm{~d}, J=9.2 \mathrm{~Hz}, 1 \mathrm{H}), 8.75(\mathrm{~d}, J=5.6 \mathrm{~Hz}, 1 \mathrm{H}), 7.95-7.90(\mathrm{~m}, 2 \mathrm{H})$, 7.80-7.70 (m, 2H), 7.51-7.45 (m, 2H), 7.37-7.30 (m, 3H); ${ }^{13} \mathrm{C}$ NMR (100 MHz, $\left.\mathrm{CDCl}_{3}\right) \delta 164.3$, $150.8,147.2,141.6,136.9,130.6,129.4,129.0,127.1,126.1,126.0,124.7,121.7$ (one peak is missing because of overlap); HRMS (ESI) $\mathrm{m} / z$ calcd for $\mathrm{C}_{16} \mathrm{H}_{12} \mathrm{NO}_{2}{ }^{+}[\mathrm{M}+\mathrm{H}]^{+}: 250.0863$ found 250.0862 . 


\section{Pd-Catalyzed Decarbonylative Cross-Coupling}

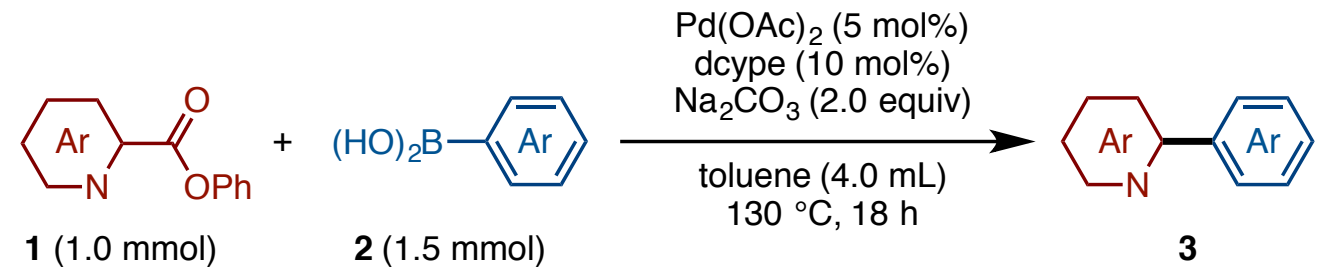

A 50-mL glass Schlenk tube containing a magnetic stirring bar was dried with a heatgun for $3 \mathrm{~min}$ in vacuo and filled with argon after cooling to room temperature. $\mathrm{Pd}(\mathrm{OAc})_{2}(11.2 \mathrm{mg}, 0.050 \mathrm{mmol}, 5$ mol\%), 1,2-bis(dicyclohexylphosphino)ethane (dcype: $42.3 \mathrm{mg}, 0.10 \mathrm{mmol}, 10 \mathrm{~mol} \%$ ), $\mathrm{Na}_{2} \mathrm{CO}_{3}(212.0$ mg, $2.0 \mathrm{mmol}, 2.0$ equiv), 1 (1.0 mmol, 1.0 equiv), and $\mathbf{2}$ (1.5 mmol, 1.5 equiv) were placed in the Schlenk tube under an argon atmosphere. Then, this tube was added dry degassed toluene $(4.0 \mathrm{~mL})$ and equipped reflux condenser under a stream of argon. The reaction mixture was stirred at $130{ }^{\circ} \mathrm{C}$ in an oil bath for $18 \mathrm{~h}$. After cooling the reaction mixture to room temperature, the mixture was diluted with EtOAc and pathed through a pad of Celite ${ }^{\circledR}$. The filtrate was concentrated in vacuo and the residue was purified by flash silica-gel column chromatography to afford biaryl $\mathbf{3}$.

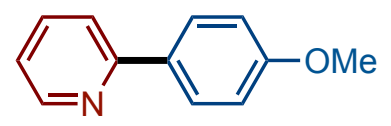

3Aa

$92 \%$

2-(4-Methoxyphenyl)pyridine $(\mathbf{3 A a})^{3}$ : Purification by flash silica-gel column chromatography (hexane/ether $=2: 1$ to 1:1) afforded 3Aa as a white solid (170.4 mg, 92\% yield). ${ }^{1} \mathrm{H}$ NMR (400 MHz, $\left.\mathrm{CDCl}_{3}\right): \delta 8.65(\mathrm{~d}, J=4.8 \mathrm{~Hz}, 1 \mathrm{H}), 7.95(\mathrm{dt}, J=8.4,2.4 \mathrm{~Hz}, 2 \mathrm{H}), 7.78-7.63(\mathrm{~m}, 2 \mathrm{H}), 7.17(\mathrm{dd}, J=6.4$, $4.8 \mathrm{~Hz}, 1 \mathrm{H}), 7.00(\mathrm{dt}, J=8.4,2.4 \mathrm{~Hz}, 2 \mathrm{H}), 3.86(\mathrm{~s}, 3 \mathrm{H}) ;{ }^{13} \mathrm{C} \mathrm{NMR}\left(100 \mathrm{MHz}, \mathrm{CDCl}_{3}\right)$ : $\delta 160.4,157.1$, 149.5, 136.6, 132.0, 128.1, 121.4, 119.8, 114.1, 55.4; HRMS (DART) $\mathrm{m} / z$ calcd for $\mathrm{C}_{12} \mathrm{H}_{12} \mathrm{NO}$ $[\mathrm{M}+\mathrm{H}]^{+}:$186.0919, found 186.0917 .

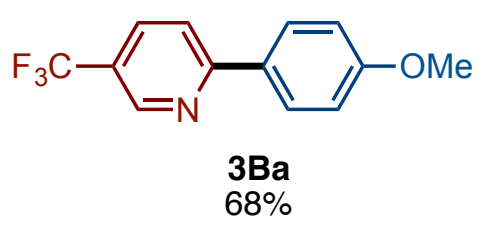

2-(4-Methoxyphenyl)-5-(trifluoromethyl)pyridine (3Ba) ${ }^{4}: 0.75 \mathrm{mmol}$ scale. Purification by flash silica-gel column chromatography (hexane/ether $=9: 1)$ afforded 3Ba as a white solid (129.2 $\mathrm{mg}, 68 \%$ yield). ${ }^{1} \mathrm{H}$ NMR (400 MHz, $\left.\mathrm{CDCl}_{3}\right): \delta 8.89(\mathrm{~s}, 1 \mathrm{H}), 8.01(\mathrm{~d}, J=8.8 \mathrm{~Hz}, 2 \mathrm{H}), 7.93(\mathrm{~d}, J=8.4 \mathrm{~Hz}, 1 \mathrm{H})$, $7.78(\mathrm{~d}, J=8.4 \mathrm{~Hz}, 1 \mathrm{H}), 7.02(\mathrm{~d}, J=8.8 \mathrm{~Hz}, 2 \mathrm{H}), 3.88(\mathrm{~s}, 3 \mathrm{H}) ;{ }^{13} \mathrm{C} \mathrm{NMR}\left(100 \mathrm{MHz}, \mathrm{CDCl}_{3}\right): \delta 161.3$, $160.2,146.5\left(\mathrm{q}, J_{C-F}=4.0 \mathrm{~Hz}\right), 133.8\left(\mathrm{q}, J_{C-F}=4.0 \mathrm{~Hz}\right), 130.5,128.6,124.0\left(\mathrm{q}, J_{C-F}=34.0 \mathrm{~Hz}\right), 123.9$ 
$\left(\mathrm{q}, J_{C-F}=270.0 \mathrm{~Hz}\right), 119.0,114.3,55.4 ;$ HRMS (DART) $\mathrm{m} / \mathrm{z}$ calcd for $\mathrm{C}_{13} \mathrm{H}_{11} \mathrm{~F}_{3} \mathrm{NO}[\mathrm{M}+\mathrm{H}]^{+}: 254.0793$, found 254.0801 .

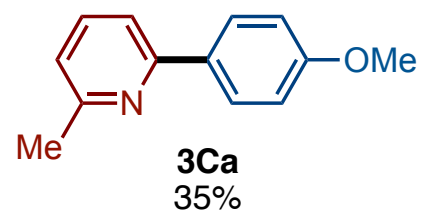

2-(4-Methoxyphenyl)-6-methylpyridine ${\left(\mathbf{3 C a}^{5}\right.}^{5}$ : Purification by flash silica-gel column chromatography (hexane/ether $=3: 1$ ) afforded $\mathbf{3 C a}$ as a white solid $\left(69.4 \mathrm{mg}, 35 \%\right.$ yield). ${ }^{1} \mathrm{H}$ NMR (400 MHz, $\left.\mathrm{CDCl}_{3}\right): \delta 7.94(\mathrm{~d}, J=8.8 \mathrm{~Hz}, 2 \mathrm{H}), 7.59(\mathrm{dd}, J=8.0,7.6 \mathrm{~Hz}, 1 \mathrm{H}), 7.45(\mathrm{~d}, J=8.0 \mathrm{~Hz}, 1 \mathrm{H})$, $7.04(\mathrm{~d}, J=7.6 \mathrm{~Hz}, 1 \mathrm{H}), 6.98(\mathrm{~d}, J=8.8 \mathrm{~Hz}, 2 \mathrm{H}), 3.86(\mathrm{~s}, 3 \mathrm{H}), 2.61(\mathrm{~s}, 3 \mathrm{H}) ;{ }^{13} \mathrm{C} \mathrm{NMR}(100 \mathrm{MHz}$, $\left.\mathrm{CDCl}_{3}\right): \delta 160.3,158.2,156.6,136.8,132.4,128.2,120.9,116.8,114.0,55.3,24.7$; HRMS (DART) $\mathrm{m} / \mathrm{z}$ calcd for $\mathrm{C}_{13} \mathrm{H}_{14} \mathrm{NO}[\mathrm{M}+\mathrm{H}]^{+}: 200.1075$, found 200.1078 .

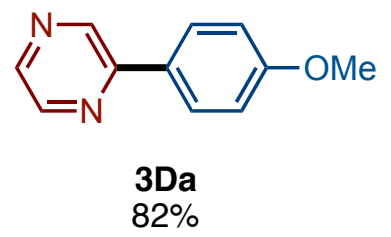

2-(4-Methoxyphenyl)pyrazine $(3 \mathrm{Da})^{6}$ : Purification by flash silica-gel column chromatography (hexane/ether $=1: 1)$ afforded 3Da as a pale brown solid $\left(152.7 \mathrm{mg}, 82 \%\right.$ yield). ${ }^{1} \mathrm{H} \mathrm{NMR}(400 \mathrm{MHz}$, $\left.\mathrm{CDCl}_{3}\right): \delta 8.98(\mathrm{~d}, J=1.2 \mathrm{~Hz}, 1 \mathrm{H}), 8.59(\mathrm{dd}, J=2.4,1.2 \mathrm{~Hz}, 1 \mathrm{H}), 8.44(\mathrm{~d}, J=2.4 \mathrm{~Hz}, 1 \mathrm{H}), 7.99(\mathrm{~d}, J$ $=8.8 \mathrm{~Hz}, 2 \mathrm{H}), 7.04(\mathrm{~d}, J=8.8 \mathrm{~Hz}, 2 \mathrm{H}), 3.89(\mathrm{~s}, 3 \mathrm{H}) ;{ }^{13} \mathrm{C} \mathrm{NMR}\left(100 \mathrm{MHz}, \mathrm{CDCl}_{3}\right): \delta 161.2,152.5$, 144.0, 142.1 141.6, 128.8, 128.3, 114.5, 55.4; HRMS (DART) $\mathrm{m} / z$ calcd for $\mathrm{C}_{11} \mathrm{H}_{11} \mathrm{~N}_{2} \mathrm{O}[\mathrm{M}+\mathrm{H}]^{+}$: 187.0871 , found 187.0876 .

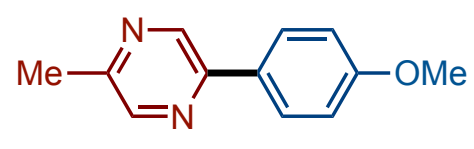

$3 E a$

2-(4-Methoxyphenyl)-5-methylpyrazine (3Ea): Purification by flash silica-gel column chromatography (hexane/ether $=1: 1)$ afforded $\mathbf{3 E a}$ as a white solid $\left(172.2 \mathrm{mg}, 86 \%\right.$ yield). ${ }^{1} \mathrm{H}$ NMR (400 MHz, $\left.\mathrm{CDCl}_{3}\right): \delta 8.85(\mathrm{~d}, J=1.2 \mathrm{~Hz}, 1 \mathrm{H}), 8.46(\mathrm{~d}, J=1.2 \mathrm{~Hz}, 1 \mathrm{H}), 7.94(\mathrm{~d}, J=8.8 \mathrm{~Hz}, 2 \mathrm{H}), 7.02$ $(\mathrm{d}, J=8.8 \mathrm{~Hz}, 2 \mathrm{H}), 3.88(\mathrm{~s}, 3 \mathrm{H}), 2.59$ (s, 3H); ${ }^{13} \mathrm{C} \mathrm{NMR}\left(100 \mathrm{MHz}, \mathrm{CDCl}_{3}\right): \delta 160.8,151.0,149.6$, 143.6, 140.4, 129.1, 128.0, 114.4, 55.4, 21.1; HRMS (DART) $\mathrm{m} / z$ calcd for $\mathrm{C}_{12} \mathrm{H}_{13} \mathrm{~N}_{2} \mathrm{O}[\mathrm{M}+\mathrm{H}]^{+}$: 201.1028 , found 201.1029 .

5. Ackermann, L.; Potukuchi, H. K.; Kapdi, A. R.; Schulzke, C. Chem. Eur. J. 2010, 16, 3300.

6. Hirner, J. J.; Blum, S. A. Organometallics 2011, 30, 1299. 


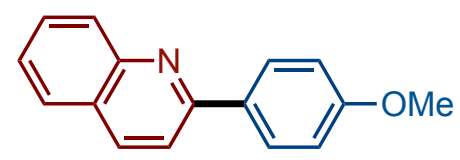

3Fa

$37 \%$

2-(4-Methoxyphenyl)quinoline $(\mathbf{3 F a})^{3}$ : Purification by flash silica-gel column chromatography (hexane/ether $=4: 1$ to $3: 1$ ) afforded $\mathbf{3 F a}$ as a white solid $\left(87.4 \mathrm{mg}, 37 \%\right.$ yield). ${ }^{1} \mathrm{H}$ NMR (400 MHz, $\left.\mathrm{CDCl}_{3}\right): \delta 8.19(\mathrm{~d}, J=8.0 \mathrm{~Hz}, 1 \mathrm{H}), 8.16-8.11(\mathrm{~m}, 3 \mathrm{H}), 7.84(\mathrm{~d}, J=8.0 \mathrm{~Hz}, 1 \mathrm{H}), 7.81(\mathrm{~d}, J=8.0 \mathrm{~Hz}$, $1 \mathrm{H}), 7.71(\mathrm{td}, J=8.0,1.2 \mathrm{~Hz}, 1 \mathrm{H}), 7.50(\mathrm{td}, J=8.0,1.2 \mathrm{~Hz}, 1 \mathrm{H}), 7.05(\mathrm{~d}, J=8.8 \mathrm{~Hz}, 2 \mathrm{H}), 3.89(\mathrm{~s}$, $3 \mathrm{H}) ;{ }^{13} \mathrm{C}$ NMR $\left(100 \mathrm{MHz}, \mathrm{CDCl}_{3}\right): \delta 160.8,156.9,148.3,136.6,132.2,129.53,129.49,128.8,127.4$, $126.9,125.9,118.5,114.2,55.4$; HRMS (DART) $\mathrm{m} / z$ calcd for $\mathrm{C}_{16} \mathrm{H}_{14} \mathrm{NO}[\mathrm{M}+\mathrm{H}]^{+}: 236.1075$, found 236.1076 .

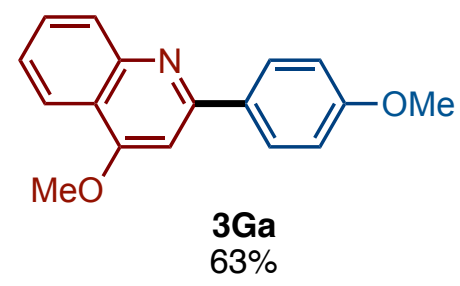

4-Methoxy-2-(4-methoxyphenyl)quinoline (3Ga) ${ }^{7}$ : $0.75 \mathrm{mmol}$ scale. Purification by flash silica-gel column chromatography (hexane/ether $=3: 1$ to 1:1) gave a mixture of $\mathbf{3 G a}$ and small amount of $\mathbf{1 G}$. As further purification, the mixture was diluted with ether and washed with $1 \mathrm{M} \mathrm{NaOHaq}$ to afford 3Ga as a yellow solid (126.3 mg, 63\% yield). ${ }^{1} \mathrm{H}$ NMR (400 MHz, $\left.\mathrm{CDCl}_{3}\right): \delta 8.17(\mathrm{dd}, J=8.0,1.2 \mathrm{~Hz}$, $1 \mathrm{H}), 8.10(\mathrm{~d}, J=8.4 \mathrm{~Hz}, 2 \mathrm{H}), 8.07(\mathrm{~d}, J=8.0 \mathrm{~Hz}, 1 \mathrm{H}), 7.69(\mathrm{td}, J=8.0,1.2 \mathrm{~Hz}, 1 \mathrm{H}), 7.46(\mathrm{td}, J=8.0$, $1.2 \mathrm{~Hz}, 1 \mathrm{H}), 7.14(\mathrm{~s}, 1 \mathrm{H}), 7.04(\mathrm{~d}, J=8.4 \mathrm{~Hz}, 2 \mathrm{H}), 4.12(\mathrm{~s}, 3 \mathrm{H}), 3.89(\mathrm{~s}, 3 \mathrm{H}) ;{ }^{13} \mathrm{C}$ NMR $(100 \mathrm{MHz}$, $\left.\mathrm{CDCl}_{3}\right): \delta 162.7,160.7,158.3,149.2,132.9,129.9,129.0,128.8,125.0,121.6,120.2,114.1,97.4$, 55.6, 55.4; HRMS (DART) $m / z$ calcd for $\mathrm{C}_{17} \mathrm{H}_{16} \mathrm{NO}_{2}[\mathrm{M}+\mathrm{H}]^{+}: 266.1181$, found 266.1181 .

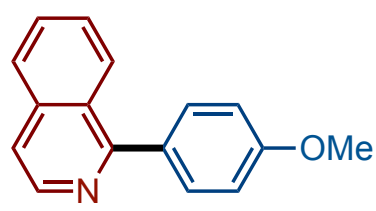

$$
\begin{aligned}
& 3 \mathrm{Ha} \\
& 53 \%
\end{aligned}
$$

1-(4-Methoxyphenyl)isoquinoline $(3 \mathrm{Ha})^{8}$ : Purification by flash silica-gel column chromatography (hexane/ether $=2: 1$ to 1:1) afforded $\mathbf{3 H a}$ as a brown solid (124.7 mg, 53\% yield). ${ }^{1} \mathrm{H}$ NMR (400 MHz,

7. Wang, Y.; Peng, C.; Liu, L.; Zhao, J.; Su, L.; Zhu, Q. Tetrahedron Lett. 2009, 50, 2261.

8. Lee, H. W.; Lam, F. L.; So, C. M.; Lau, C. P.; Chan, A. S. C.; Kwong, F. Y. Angew. Chem., Int. Ed. 2009, 48, 7436. 
$\left.\mathrm{CDCl}_{3}\right): \delta 8.59(\mathrm{~d}, J=6.0 \mathrm{~Hz}, 1 \mathrm{H}), 8.15(\mathrm{~d}, J=7.6 \mathrm{~Hz}, 1 \mathrm{H}), 7.88(\mathrm{~d}, J=8.4 \mathrm{~Hz}, 1 \mathrm{H}), 7.72-7.64(\mathrm{~m}$, $3 \mathrm{H}), 7.62(\mathrm{~d}, J=6.0 \mathrm{~Hz}, 1 \mathrm{H}), 7.57-7.51(\mathrm{~m}, 1 \mathrm{H}), 7.07(\mathrm{~d}, J=8.0 \mathrm{~Hz}, 2 \mathrm{H}), 3.91(\mathrm{~s}, 3 \mathrm{H}) ;{ }^{13} \mathrm{C}$ NMR $\left(100 \mathrm{MHz}, \mathrm{CDCl}_{3}\right): \delta 160.3,156.0,142.2,136.9,132.1,131.2,129.8,127.6,127.0,126.9,126.7$, 119.5, 113.7, 55.3; HRMS (DART) $\mathrm{m} / z$ calcd for $\mathrm{C}_{16} \mathrm{H}_{14} \mathrm{NO}[\mathrm{M}+\mathrm{H}]^{+}: 236.1075$, found 236.1079.

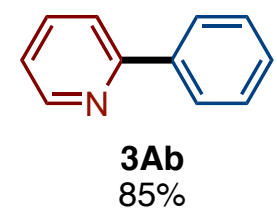

2-Phenylpyridine (3Ab) ${ }^{9}$ : Purification by flash silica-gel column chromatography (hexane/ether = 3:1) afforded $\mathbf{3 A b}$ as a pale yellow oil (131.9 mg, 85\% yield). ${ }^{1} \mathrm{H}$ NMR (400 MHz, $\left.\mathrm{CDCl}_{3}\right): \delta 8.70$ (d, $J=5.2 \mathrm{~Hz}, 1 \mathrm{H}), 7.99(\mathrm{~d}, J=8.0 \mathrm{~Hz}, 2 \mathrm{H}), 7.81-7.69(\mathrm{~m}, 2 \mathrm{H}), 7.48(\mathrm{dd}, J=8.0,7.2 \mathrm{~Hz}, 2 \mathrm{H}), 7.42(\mathrm{t}, J$ $=7.2 \mathrm{~Hz}, 1 \mathrm{H}), 7.26-7.21(\mathrm{~m}, 1 \mathrm{H}) ;{ }^{13} \mathrm{C} \mathrm{NMR}\left(100 \mathrm{MHz}, \mathrm{CDCl}_{3}\right): \delta 157.4,149.6,139.4,136.7,128.9$, 128.7, 126.9, 122.0, 120.5; HRMS (DART) $m / z$ calcd for $\mathrm{C}_{11} \mathrm{H}_{10} \mathrm{~N}[\mathrm{M}+\mathrm{H}]^{+}$: 156.0813, found 156.0817.

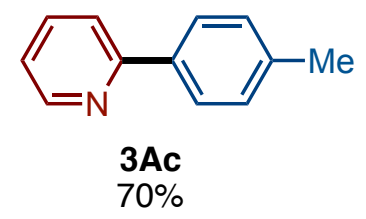

2-( $\boldsymbol{p}$-Tolyl)pyridine (3Aq) ${ }^{9}$ : Purification by flash silica-gel column chromatography (hexane/ether = 3:1) afforded 3Ac as a colorless oil (118.5 mg, 70\% yield). ${ }^{1} \mathrm{H}$ NMR (400 MHz, $\left.\mathrm{CDCl}_{3}\right): \delta 8.68(\mathrm{~d}, J=$ $4.4 \mathrm{~Hz}, 1 \mathrm{H}), 7.89$ (d, $J=8.4 \mathrm{~Hz}, 2 \mathrm{H}), 7.82-7.65(\mathrm{~m}, 2 \mathrm{H}), 7.29$ (d, $J=8.4 \mathrm{~Hz}, 2 \mathrm{H}), 7.20$ (dd, $J=7.6$, $4.4 \mathrm{~Hz}, 1 \mathrm{H}), 2.41(\mathrm{~s}, 3 \mathrm{H}) ;{ }^{13} \mathrm{C} \mathrm{NMR}\left(100 \mathrm{MHz}, \mathrm{CDCl}_{3}\right)$ : $\delta 157.4,149.5,138.9,136.61,136.56,129.4$, 126.7, 121.7, 120.2, 21.2; HRMS (DART) $\mathrm{m} / z$ calcd for $\mathrm{C}_{12} \mathrm{H}_{12} \mathrm{~N}[\mathrm{M}+\mathrm{H}]^{+}:$170.0970, found 170.0978.

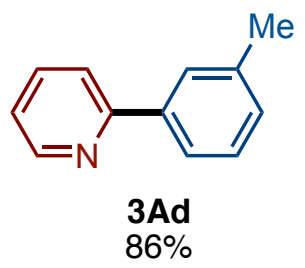

2-( $\boldsymbol{m}$-Tolyl)pyridine (3Ad $)^{9}$ : Purification by flash silica-gel column chromatography (hexane/ether = 3:1) afforded 3Ad as a white solid (145.5 mg, 86\% yield). ${ }^{1} \mathrm{H}$ NMR (400 MHz, $\left.\mathrm{CDCl}_{3}\right): \delta 8.70(\mathrm{~d}, J=$ $5.2 \mathrm{~Hz}, 1 \mathrm{H}), 7.84(\mathrm{~s}, 1 \mathrm{H}), 7.79-7.69(\mathrm{~m}, 3 \mathrm{H}), 7.37(\mathrm{dd}, J=8.0,7.8 \mathrm{~Hz}, 1 \mathrm{H}), 7.25-7.21(\mathrm{~m}, 2 \mathrm{H}), 2.44$ (s, 3H); ${ }^{13} \mathrm{C}$ NMR $\left(100 \mathrm{MHz}, \mathrm{CDCl}_{3}\right): \delta$ 157.6, 149.6, 139.3, 138.4, 136.7, 129.7, 128.6, 127.6, 124.0, 122.0, 120.6, 21.5; HRMS (DART) $\mathrm{m} / z$ calcd for $\mathrm{C}_{12} \mathrm{H}_{12} \mathrm{~N}[\mathrm{M}+\mathrm{H}]^{+}: 170.0970$, found 170.0968 .

9. Hu, B.; Li, Y.; Dong, W. Xie, X.; Wan, J.; Zhang, Z. RSC Adv. 2016, 6, 48315. 


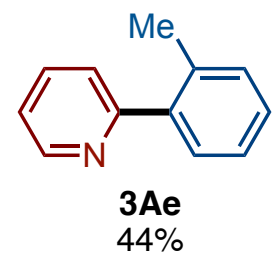

2-(o-Tolyl)pyridine (3Ae) ${ }^{9}$ : Purification by flash silica-gel column chromatography (hexane/ether = 3:1) afforded 3Ae as a colorless oil $\left(74.7 \mathrm{mg}, 44 \%\right.$ yield). ${ }^{1} \mathrm{H}$ NMR (400 MHz, $\left.\mathrm{CDCl}_{3}\right): \delta 8.70(\mathrm{~d}, J=$ $4.4 \mathrm{~Hz}, 1 \mathrm{H}), 7.75(\mathrm{td}, J=8.0,2.0 \mathrm{~Hz}, 1 \mathrm{H}), 7.43-7.37$ (m, 2H), 7.34-7.19 (m, 4H), $2.37(\mathrm{~s}, 3 \mathrm{H}) ;{ }^{13} \mathrm{C}$ NMR $\left(100 \mathrm{MHz}, \mathrm{CDCl}_{3}\right): \delta 160.0,149.1,140.4,136.1,135.7,130.7,129.6,128.2,125.8,124.1$, 121.6, 20.2; HRMS (DART) $\mathrm{m} / z$ calcd for $\mathrm{C}_{12} \mathrm{H}_{12} \mathrm{~N}[\mathrm{M}+\mathrm{H}]^{+}: 170.0970$, found 170.0973 .

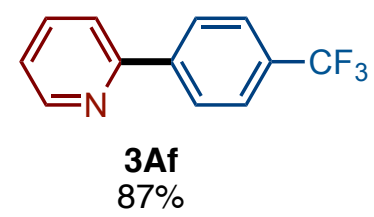

2-(4-(Trifluoromethyl)phenyl)pyridine (3Af)': Purification by flash silica-gel column chromatography (hexane/ether $=4: 1)$ afforded 3Af as a white solid $\left(194.2 \mathrm{mg}, 87 \%\right.$ yield). ${ }^{1} \mathrm{H}$ NMR $\left(400 \mathrm{MHz}, \mathrm{CDCl}_{3}\right): \delta 8.73(\mathrm{~d}, J=5.2 \mathrm{~Hz}, 1 \mathrm{H}), 8.12(\mathrm{~d}, J=8.0 \mathrm{~Hz}, 2 \mathrm{H}), 7.85-7.75(\mathrm{~m}, 2 \mathrm{H}), 7.74(\mathrm{~d}, J$ $=8.0 \mathrm{~Hz}, 2 \mathrm{H}), 7.31(\mathrm{dd}, J=7.2,5.2 \mathrm{~Hz}, 1 \mathrm{H}) ;{ }^{13} \mathrm{C} \mathrm{NMR}\left(100 \mathrm{MHz}, \mathrm{CDCl}_{3}\right): \delta 155.9,149.9,142.7$, $137.0,130.8\left(\mathrm{q}, J_{C-F}=31.4 \mathrm{~Hz}\right), 127.2,125.7,124.7\left(\mathrm{q}, J_{C-F}=271 \mathrm{~Hz}\right), 122.9,120.8$; HRMS (DART) $\mathrm{m} / \mathrm{z}$ calcd for $\mathrm{C}_{12} \mathrm{H}_{9} \mathrm{~F}_{3} \mathrm{~N}[\mathrm{M}+\mathrm{H}]^{+}: 224.0687$, found 224.0685 .

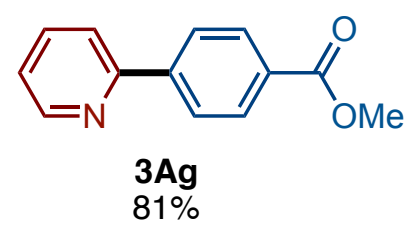

Methyl 4-(pyridin-2-yl)benzoate (3Ag) ${ }^{10}$ : Purification by flash silica-gel column chromatography (hexane/ether $=2: 1$ to $1: 1)$ afforded $\mathbf{3 A g}$ as a white solid (174.4 mg, 81\% yield). ${ }^{1} \mathrm{H}$ NMR (400 MHz, $\left.\mathrm{CDCl}_{3}\right): \delta 8.73(\mathrm{~d}, J=4.8 \mathrm{~Hz}, 1 \mathrm{H}), 8.15(\mathrm{~d}, J=8.0 \mathrm{~Hz}, 2 \mathrm{H}), 8.08(\mathrm{~d}, J=8.0 \mathrm{~Hz}, 2 \mathrm{H}), 7.80(\mathrm{dd}, J=4.8$, $1.2 \mathrm{~Hz}, 2 \mathrm{H}), 7.29(\mathrm{dd}, J=8.0,4.8 \mathrm{~Hz}, 1 \mathrm{H}), 3.95(\mathrm{~s}, 3 \mathrm{H}) ;{ }^{13} \mathrm{C} \mathrm{NMR}\left(100 \mathrm{MHz}, \mathrm{CDCl}_{3}\right): \delta$ 166.9, 156.2, $149.9,143.5,136.9,130.3,130.0,126.8,122.8,121.0,52.2$; HRMS (DART) $\mathrm{m} / z$ calcd for $\mathrm{C}_{13} \mathrm{H}_{12} \mathrm{NO}_{2}$ $[\mathrm{M}+\mathrm{H}]^{+}: 214.0868$, found 214.0873 .

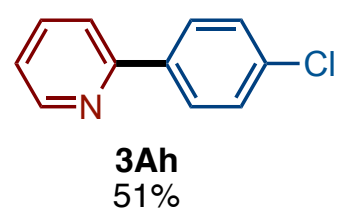

10. Luzung, M. R.; Patel, J. S.; Yin, J. J. Org. Chem. 2010, 75, 8330. 
2-(4-Chlorophenyl)pyridine (3Ah) ${ }^{9}$ : Purification by flash silica-gel column chromatography (hexane/ether $=4: 1)$ afforded $\mathbf{3 A h}$ as a white solid $\left(96.7 \mathrm{mg}, 51 \%\right.$ yield). ${ }^{1} \mathrm{H}$ NMR $\left(400 \mathrm{MHz}, \mathrm{CDCl}_{3}\right)$ : $\delta 8.69(\mathrm{~d}, J=5.2 \mathrm{~Hz}, 1 \mathrm{H}), 7.94(\mathrm{~d}, J=8.8 \mathrm{~Hz}, 2 \mathrm{H}), 7.76(\mathrm{dd}, J=8.0,7.2 \mathrm{~Hz}, 1 \mathrm{H}), 7.70(\mathrm{~d}, J=8.0 \mathrm{~Hz}$, $1 \mathrm{H}), 7.45(\mathrm{~d}, J=8.8 \mathrm{~Hz}, 2 \mathrm{H}), 7.23(\mathrm{dd}, J=7.2,5.2 \mathrm{~Hz}, 1 \mathrm{H}) ;{ }^{13} \mathrm{C} \mathrm{NMR}\left(100 \mathrm{MHz}, \mathrm{CDCl}_{3}\right): \delta 156.2$, $149.7,137.8,136.8,135.0,128.90,128.86,128.1,122.3,120.3$; HRMS (DART) $\mathrm{m} / \mathrm{z}$ calcd for $\mathrm{C}_{11} \mathrm{H}_{9} \mathrm{ClN}[\mathrm{M}+\mathrm{H}]^{+}: 190.0418$, found 190.0417 .

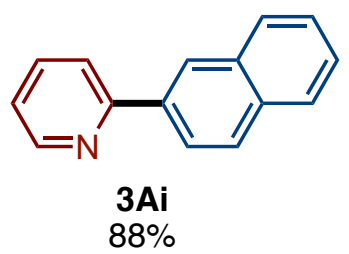

2-(Naphthalen-2-yl)pyridine $(\mathbf{3 A i})^{11}$ : Purification by flash silica-gel column chromatography (hexane/ether $=3: 1)$ afforded $\mathbf{3 A h}$ as a white solid $\left(180.6 \mathrm{mg}, 88 \%\right.$ yield). ${ }^{1} \mathrm{H}$ NMR $(400 \mathrm{MHz}$, $\left.\mathrm{CDCl}_{3}\right): \delta 8.75(\mathrm{~d}, J=4.8 \mathrm{~Hz}, 1 \mathrm{H}), 8.49(\mathrm{~s}, 1 \mathrm{H}), 8.14(\mathrm{dd}, J=8.4,2.0 \mathrm{~Hz}, 1 \mathrm{H}), 7.98-7.92(\mathrm{~m}, 2 \mathrm{H})$, $7.90-7.85(\mathrm{~m}, 2 \mathrm{H}), 7.80(\mathrm{td}, J=8.4,2.0 \mathrm{~Hz}, 1 \mathrm{H}), 7.54-7.48(\mathrm{~m}, 2 \mathrm{H}), 7.29-7.23(\mathrm{~m}, 1 \mathrm{H}) ;{ }^{13} \mathrm{C} \mathrm{NMR}$ $\left(100 \mathrm{MHz}, \mathrm{CDCl}_{3}\right): \delta 157.3,149.7,136.8,136.6,133.6,133.5,128.7,128.4,127.6,126.5,126.4$, 126.3, 124.5, 122.1, 120.8; HRMS (DART) $\mathrm{m} / z$ calcd for $\mathrm{C}_{15} \mathrm{H}_{12} \mathrm{~N}[\mathrm{M}+\mathrm{H}]^{+}: 206.0970$, found 206.0960.
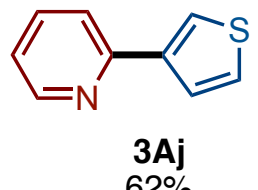

$62 \%$

2-(Thiophen-3-yl)pyridine $(\mathbf{3 A i})^{10}$ : Purification by flash silica-gel column chromatography (hexane/ether = 3:1) afforded $\mathbf{3 A i}$ as a white solid (100.0 mg, 62\% yield). ${ }^{1} \mathrm{H}$ NMR (400 $\mathrm{MHz}$, $\left.\mathrm{CDCl}_{3}\right): \delta 8.62(\mathrm{~d}, J=4.8 \mathrm{~Hz}, 1 \mathrm{H}), 7.90(\mathrm{~d}, J=2.8 \mathrm{~Hz}, 1 \mathrm{H}), 7.70(\mathrm{t}, J=8.0 \mathrm{~Hz}, 1 \mathrm{H}), 7.66(\mathrm{~d}, J=5.2$ $\mathrm{Hz}, 1 \mathrm{H}), 7.62(\mathrm{~d}, J=7.6 \mathrm{~Hz}, 1 \mathrm{H}), 7.40(\mathrm{dd}, J=5.2,2.8 \mathrm{~Hz}, 1 \mathrm{H}), 7.17(\mathrm{dd}, J=8.0,4.8 \mathrm{~Hz}, 1 \mathrm{H}) ;{ }^{13} \mathrm{C}$ NMR (100 MHz, $\mathrm{CDCl}_{3}$ ): $\delta 153.5,149.6,142.1,136.6,126.2,126.1,123.4,121.7,120.2$; HRMS (DART) $\mathrm{m} / z$ calcd for $\mathrm{C}_{9} \mathrm{H}_{8} \mathrm{NS}[\mathrm{M}+\mathrm{H}]^{+}: 162.0377$, found 162.0382 .

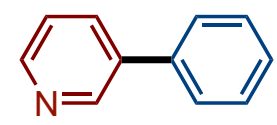

3lb

$55 \%$

3-Phenylpyridine (3Ib) ${ }^{3}: 0.40 \mathrm{mmol}$ scale. Purification by flash silica-gel column chromatography (hexane/ether $=3: 1)$ afforded 3Ib as a colorless liquid (with dcype ligand: no reaction; with $\mathrm{P}(n-\mathrm{Bu})_{3}$ ligand: $34.0 \mathrm{mg}, 55 \%$ yield). ${ }^{1} \mathrm{H} \mathrm{NMR}\left(400 \mathrm{MHz}, \mathrm{CDCl}_{3}\right): \delta 8.85$ (d, $\left.J=2.4 \mathrm{~Hz}, 1 \mathrm{H}\right), 8.58$ (dd, $J=4.8$,

11. Kobayashi, O.; Uraguchi, D.; Yamakawa, T. Org. Lett. 2009, 11, 2679. 
$2.4 \mathrm{~Hz}, 1 \mathrm{H}), 7.87(\mathrm{dd}, J=8.4,2.4 \mathrm{~Hz}, 1 \mathrm{H}), 7.58(\mathrm{~d}, J=7.2 \mathrm{~Hz}, 2 \mathrm{H}), 7.49(\mathrm{dd}, J=8.4,7.2 \mathrm{~Hz}, 2 \mathrm{H})$, $7.40(\mathrm{t}, J=8.4 \mathrm{~Hz}, 1 \mathrm{H}), 7.36(\mathrm{dd}, J=8.4,4.8 \mathrm{~Hz}, 1 \mathrm{H}) ;{ }^{13} \mathrm{C} \mathrm{NMR}\left(100 \mathrm{MHz}, \mathrm{CDCl}_{3}\right): \delta 148.3,148.2$, $137.7,136.6,134.3,129.0,128.0,127.1,123.5$; HRMS (DART) $\mathrm{m} / \mathrm{z}$ calcd for $\mathrm{C}_{11} \mathrm{H}_{10} \mathrm{~N}[\mathrm{M}+\mathrm{H}]^{+}$: 156.0808 , found 156.0806 .

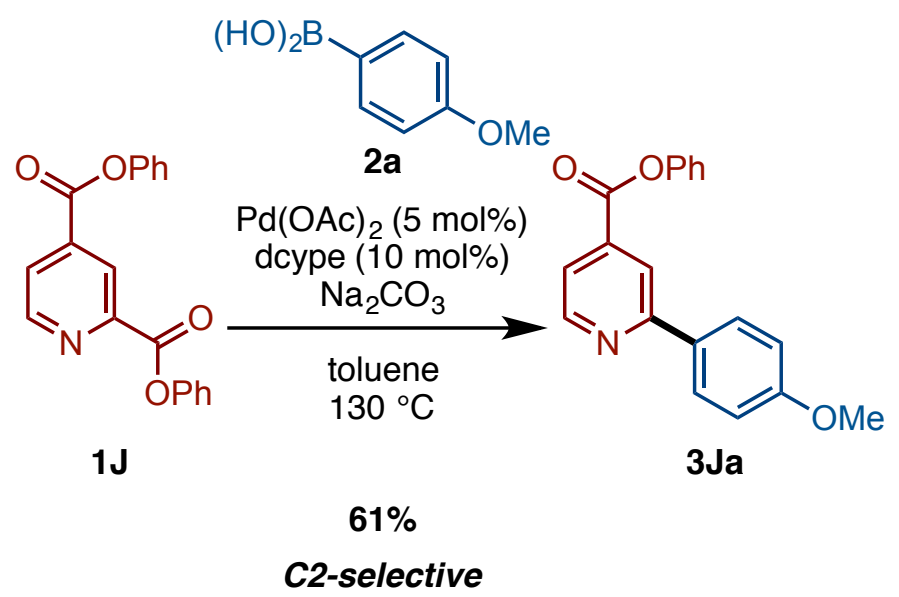

\section{Phenyl 2-(4-methoxyphenyl)isonicotinate (3Ja)}

2 mmol scale. A $50-\mathrm{mL}$ glass Schlenk tube containing a magnetic stirring bar was dried with a heatgun for $3 \mathrm{~min}$ in vacuo and filled with argon after cooling to room temperature. $\mathrm{Pd}(\mathrm{OAc})_{2}(22.5$ $\mathrm{mg}, 0.10 \mathrm{mmol}, 5 \mathrm{~mol} \%$ ), 1,2-bis(dicyclohexylphosphino)ethane (dcype: $84.5 \mathrm{mg}, 0.20 \mathrm{mmol}, 10$ mol\%), $\mathrm{Na}_{2} \mathrm{CO}_{3}$ (424.0 mg, $2.0 \mathrm{mmol}, 2.0$ equiv), diphenyl pyridine-2,4-dicarboxylate (1J: $638.6 \mathrm{mg}$, $2.0 \mathrm{mmol}, 1.0$ equiv), and (4-methoxyphenyl)boronic acid (2a: $455.9 \mathrm{mg}, 3.0 \mathrm{mmol}, 1.5$ equiv) were placed in the Schlenk tube under an argon atmosphere. Then, this tube was added dry degassed toluene $(8.0 \mathrm{~mL})$ and equipped reflux condenser under a stream of argon. The reaction mixture was stirred at $130{ }^{\circ} \mathrm{C}$ in an oil bath for $18 \mathrm{~h}$. After cooling the reaction mixture to room temperature, the mixture was diluted with EtOAc and pathed through a pad of Celite ${ }^{\circledR}$. The filtrate was concentrated in vacuo and the residue was purified by flash silica-gel column chromatography (hexane/ether $=3: 1$ to 1:1) to afford $3 \mathrm{Ja}$ as a white solid (368.1 $\mathrm{mg}, 61 \%$ yield). ${ }^{1} \mathrm{H}$ NMR (400 MHz, $\left.\mathrm{CDCl}_{3}\right): \delta 8.87(\mathrm{dd}, J=$ $4.8,1.2 \mathrm{~Hz}, 1 \mathrm{H}), 8.38(\mathrm{~d}, J=1.2 \mathrm{~Hz}, 1 \mathrm{H}), 8.06(\mathrm{~d}, J=8.4 \mathrm{~Hz}, 2 \mathrm{H}), 7.87(\mathrm{dd}, J=4.8,1.2 \mathrm{~Hz}, 1 \mathrm{H}), 7.47$ $(\mathrm{dd}, J=8.0,7.2 \mathrm{~Hz}, 2 \mathrm{H}), 7.33(\mathrm{~d}, J=7.2 \mathrm{~Hz}, 1 \mathrm{H}), 7.25(\mathrm{~d}, J=8.0 \mathrm{~Hz}, 2 \mathrm{H}), 7.04(\mathrm{~d}, J=8.4 \mathrm{~Hz}, 2 \mathrm{H})$, 3.89 (s, 3H); ${ }^{13} \mathrm{C} \mathrm{NMR}\left(100 \mathrm{MHz}, \mathrm{CDCl}_{3}\right)$ : $\delta 164.0,160.9,158.3,150.54,150.45,137.6,130.9,129.6$, 128.3, 126.3, 121.4, 120.6, 119.2, 114.2, 55.3; HRMS (DART) $\mathrm{m} / z$ calcd for $\mathrm{C}_{19} \mathrm{H}_{16} \mathrm{NO}_{3}[\mathrm{M}+\mathrm{H}]^{+}$: 306.1130 , found 306.1130 . 


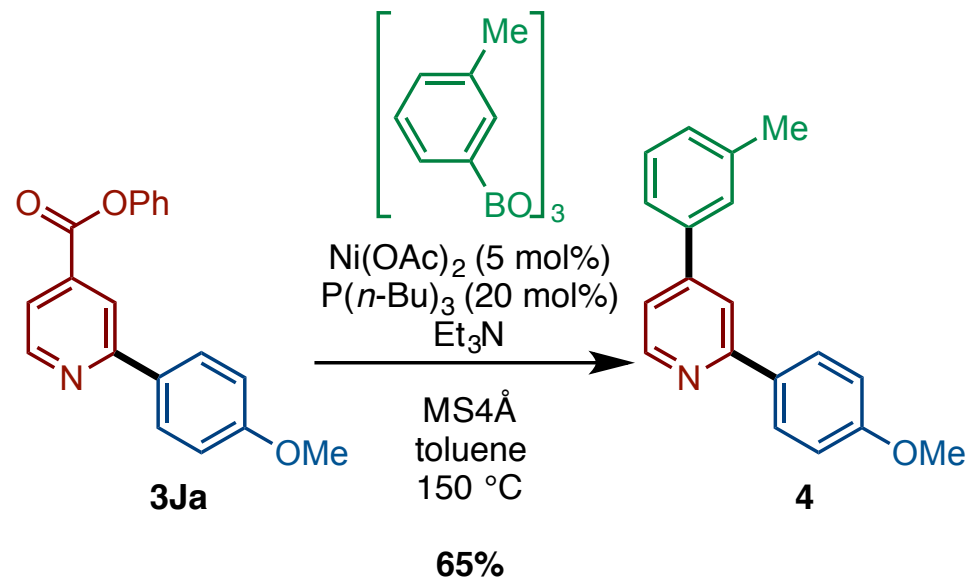

\section{2-(4-Methoxyphenyl)-4-(m-tolyl)pyridine (4)}

A $100-\mathrm{mL}$ glass $\mathrm{J}$-Young ${ }^{\circledR}$ tube containing a magnetic stirring bar and $250 \mathrm{mg}$ of molecular sieves

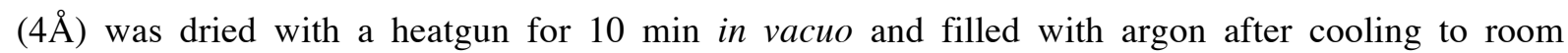
temperature. $\mathrm{Ni}(\mathrm{OAc})_{2}(8.8 \mathrm{mg}, 0.050 \mathrm{mmol}, 5 \mathrm{~mol} \%)$, phenyl 2-(4-methoxyphenyl)isonicotinate (3Ja) (305.3 mg, $1.0 \mathrm{mmol}, 1.0$ equiv), and $m$-tolyl boroxine (176.9 mg, $0.50 \mathrm{mmol}, 0.50$ equiv) were placed in the tube under an argon atmosphere. Then, this tube were added $\mathrm{P}(n-\mathrm{Bu})_{3}(40.5 \mathrm{mg}, 0.20$ mmol, $20 \mathrm{~mol} \%), \mathrm{Et}_{3} \mathrm{~N}$ (151.8 mg, $1.5 \mathrm{mmol}, 1.5$ equiv), and dry degassed toluene (4.0 mL) and sealed with O-ring tap under a stream of argon. The reaction mixture was stirred at $150{ }^{\circ} \mathrm{C}$ in an oil bath for $24 \mathrm{~h}$. After cooling the reaction mixture to room temperature, the mixture was diluted with EtOAc and pathed through a pad of Celite ${ }^{\circledast}$. The filtrate was concentrated in vacuo and the residue was purified by flash silica-gel column chromatography (hexane/ether $=5: 2$ ) to afford $\mathbf{4}$ as a colorless oil (179.0 mg, 65\% yield). ${ }^{1} \mathrm{H}$ NMR (400 MHz, $\left.\mathrm{CDCl}_{3}\right): \delta 8.68(\mathrm{~d}, J=6.0 \mathrm{~Hz}, 1 \mathrm{H}), 8.02(\mathrm{~d}, J=8.4 \mathrm{~Hz}$, 2H), $7.86(\mathrm{~s}, 1 \mathrm{H}), 7.51-7.47(\mathrm{~m}, 2 \mathrm{H}), 7.41(\mathrm{~d}, J=8.0 \mathrm{~Hz}, 1 \mathrm{H}), 7.39(\mathrm{~d}, J=6.0 \mathrm{~Hz}, 1 \mathrm{H}), 7.28(\mathrm{~d}, J=$ $8.0 \mathrm{~Hz}, 1 \mathrm{H}), 7.02(\mathrm{~d}, J=8.4 \mathrm{~Hz}, 2 \mathrm{H}), 3.88(\mathrm{~s}, 3 \mathrm{H}), 2.46(\mathrm{~s}, 3 \mathrm{H}) ;{ }^{13} \mathrm{C} \mathrm{NMR}\left(100 \mathrm{MHz}, \mathrm{CDCl}_{3}\right): \delta 160.4$, 157.5, 149.8, 149.2, 138.7, 138.6, 132.0, 129.6, 128.9, 128.2, 127.7, 124.1, 119.6, 117.9, 114.0, 55.2, 21.4; HRMS (DART) $\mathrm{m} / z$ calcd for $\mathrm{C}_{19} \mathrm{H}_{18} \mathrm{NO}[\mathrm{M}+\mathrm{H}]^{+}:$276.1388, found 276.1388 . 


\section{Condition Screening}

Effect of temperature
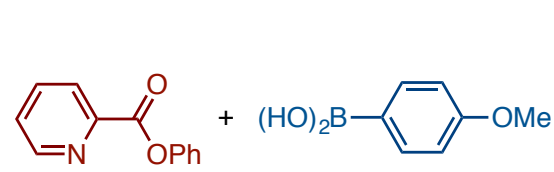

$\mathrm{Pd}(\mathrm{OAc})_{2}(5 \mathrm{~mol} \%)$

$1 \mathrm{~A}(1.0 \mathrm{mmol})$

$2 \mathbf{a}(1.5 \mathrm{mmol})$

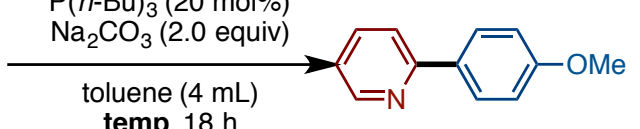

temp, $18 \mathrm{~h}$

$3 \mathrm{Aa}$<smiles>COc1ccc(C(=O)c2ccccn2)cc1</smiles>

\begin{tabular}{cccc}
\hline & & \multicolumn{2}{c}{ NMR yield/\% } \\
entry & temp $/{ }^{\circ} \mathrm{C}$ & 3Aa & S1 \\
\hline 1 & 150 & 38 & 9 \\
2 & 130 & 48 & 7 \\
3 & 100 & 8 & 8 \\
4 & 80 & 7 & 5 \\
\hline
\end{tabular}

NMR yield was detemined by using 1,4-

bis(trimethylsilyl)benzene as an internal standard.

Effect of ligand

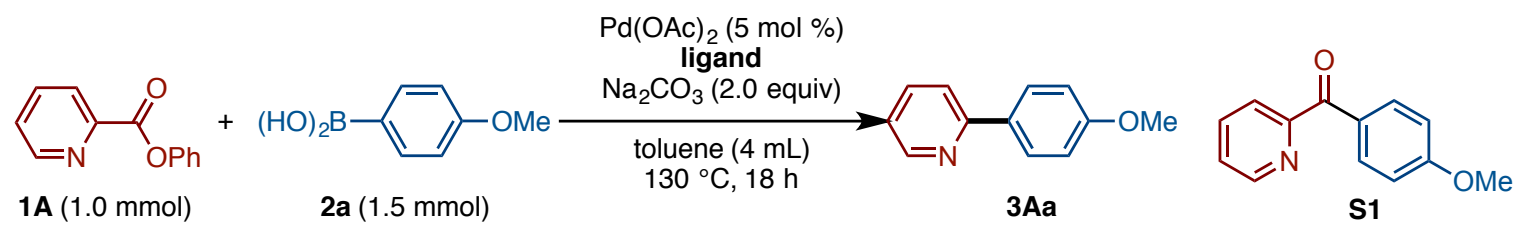

\begin{tabular}{|c|c|c|c|c|c|c|c|}
\hline \multirow[b]{2}{*}{ entry } & \multirow[b]{2}{*}{ ligand/mol\% } & \multicolumn{2}{|c|}{ NMR yield $/ \%$} & \multirow[b]{2}{*}{ entry } & \multirow[b]{2}{*}{ ligand $/ \mathrm{mol} \%$} & \multicolumn{2}{|c|}{ NMR yield/\% } \\
\hline & & 3Aa & S1 & & & 3Aa & S1 \\
\hline 1 & $\mathrm{P}(n-\mathrm{Bu})_{3} / 20$ & 48 & 7 & 11 & dcype /10 & 97 & 0 \\
\hline 2 & $\mathrm{PPh}_{3} / 20$ & 13 & 0 & 12 & dcypt /20 & 0 & 0 \\
\hline 3 & $\mathrm{P}(p \text {-Anisyl })_{3} / 20$ & 14 & 0 & 13 & dppe /10 & 11 & 0 \\
\hline 4 & $\mathrm{PCy}_{3} / 20$ & 7 & 3 & 14 & dppp /10 & 20 & 0 \\
\hline 5 & SPhos /20 & 20 & 3 & 15 & dppb /10 & 19 & 0 \\
\hline 6 & XPhos /20 & 19 & 0 & 16 & dppf /20 & 12 & 0 \\
\hline 7 & $\mathrm{P}(t-\mathrm{Bu})_{3} \cdot \mathrm{HBF}_{4} / 20$ & 0 & 0 & 17 & DPEphos & 12 & 0 \\
\hline 8 & $\mathrm{P}(t-\mathrm{Bu})_{2} \mathrm{Me} / 20$ & 16 & 3 & 18 & Xantphos & 17 & 0 \\
\hline 9 & Amphos /20 & 4 & 0 & 19 & $\mathrm{IPr} \cdot \mathrm{HCl}$ & 7 & 0 \\
\hline 10 & dcype /20 & 62 & 0 & 20 & $\mathrm{SIPr} \cdot \mathrm{HCl}$ & 7 & 0 \\
\hline
\end{tabular}

NMR yield was detemined by using 1,4-bis(trimethylsilyl)benzene as an internal standard.

Effect of base

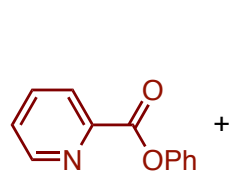

$1 \mathrm{~A}(1.0 \mathrm{mmol})$

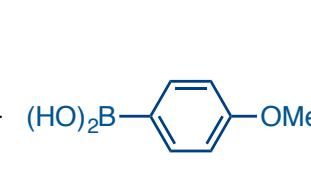

$\mathbf{2 a}(1.5 \mathrm{mmol})$

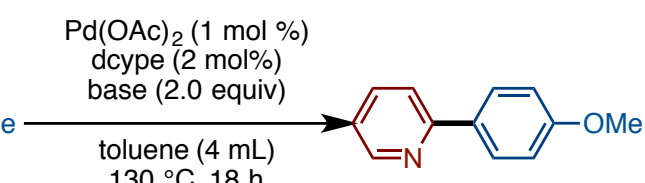

3Aa<smiles>COc1ccc(C(=O)c2ccccn2)cc1</smiles>

S1

\begin{tabular}{clcc}
\hline & & \multicolumn{2}{c}{ NMR yield/\% } \\
entry & base & 3Aa & S1 \\
\hline 1 & $\mathrm{Na}_{2} \mathrm{CO}_{3}$ & 28 & 1 \\
2 & $\mathrm{~K}_{2} \mathrm{CO}_{3}$ & 12 & 0 \\
3 & $\mathrm{NaHCO}_{3}$ & 18 & 0 \\
4 & $\mathrm{Li}_{2} \mathrm{CO}_{3}$ & 0 & 0 \\
5 & $\mathrm{~K}_{3} \mathrm{PO}_{4}$ & 19 & 0 \\
6 & $\mathrm{Et}_{3} \mathrm{~N}$ & 34 & 0 \\
\hline
\end{tabular}

NMR yield was detemined by using 1,4-

bis(trimethylsilyl)benzene as an internal standard. 


\section{5. ${ }^{1} \mathrm{H}$ NMR and ${ }^{13} \mathrm{C}$ NMR Spectra}

${ }^{1} \mathrm{H} \mathrm{NMR}$ of $1 \mathbf{B}\left(600 \mathrm{MHz}, \mathrm{CDCl}_{3}\right)$

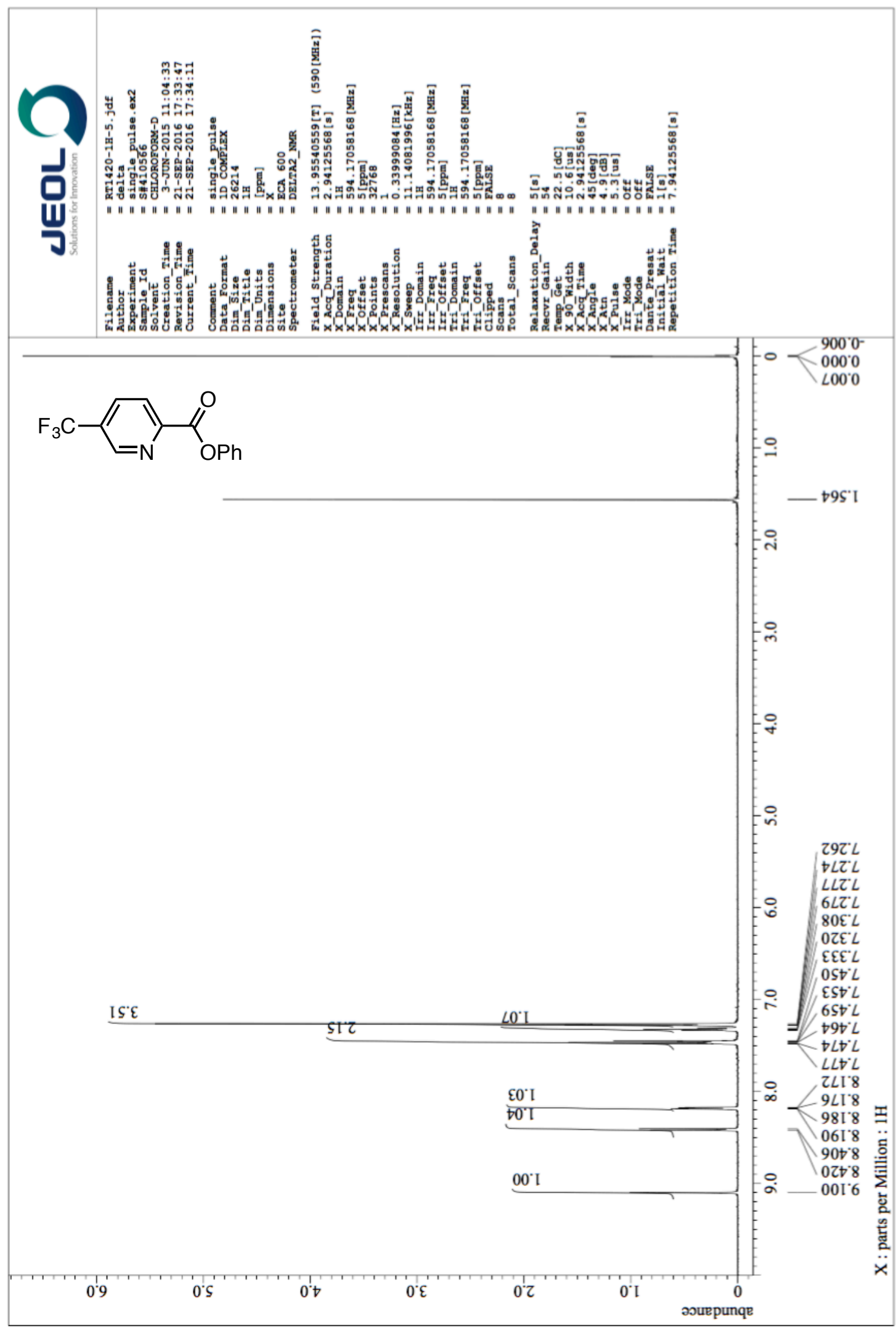


${ }^{13} \mathrm{C}$ NMR of $1 B\left(400 \mathrm{MHz}, \mathrm{CDCl}_{3}\right)$

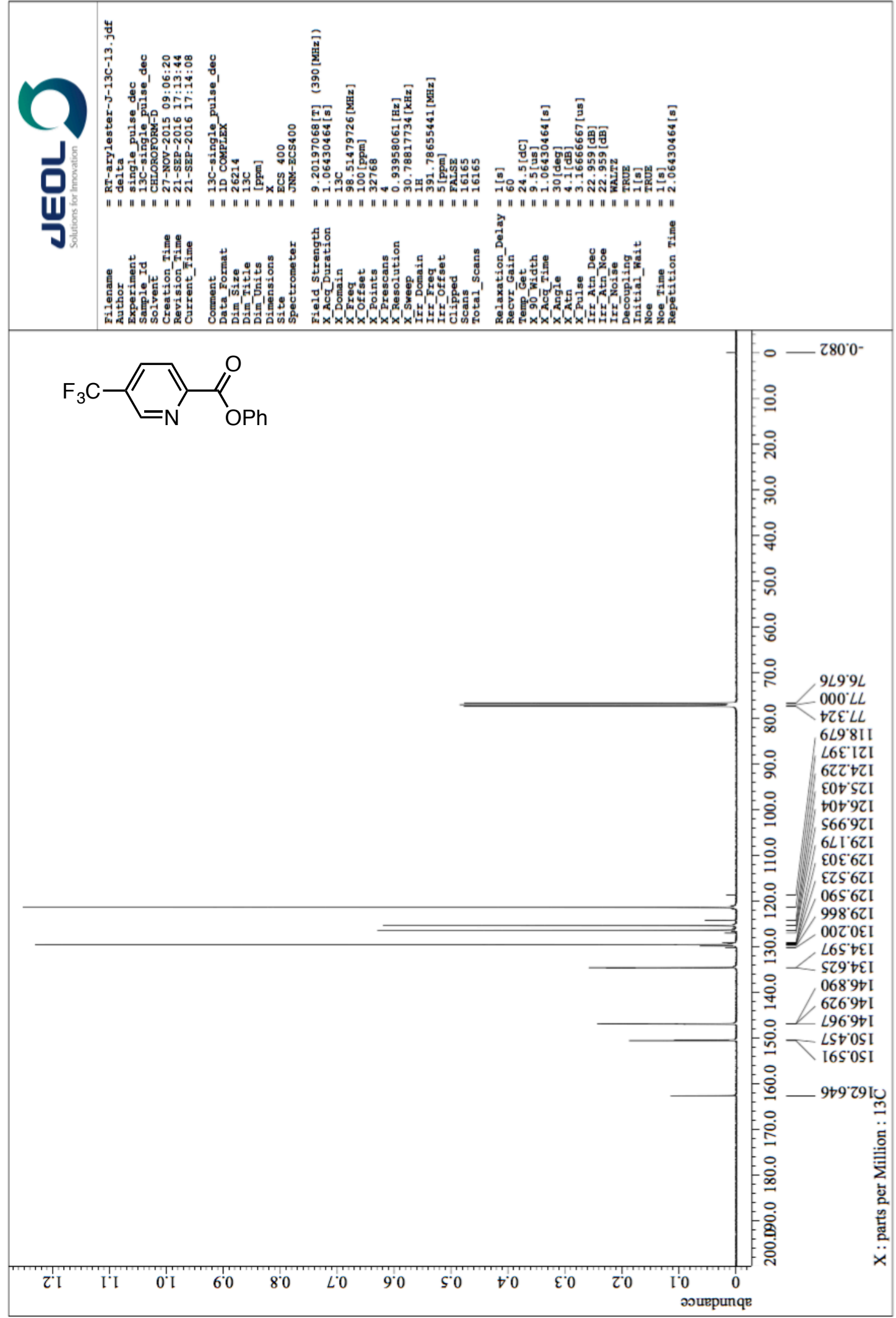


${ }^{1} \mathrm{H} \mathrm{NMR}$ of $\mathbf{1 C}\left(600 \mathrm{MHz}, \mathrm{CDCl}_{3}\right)$

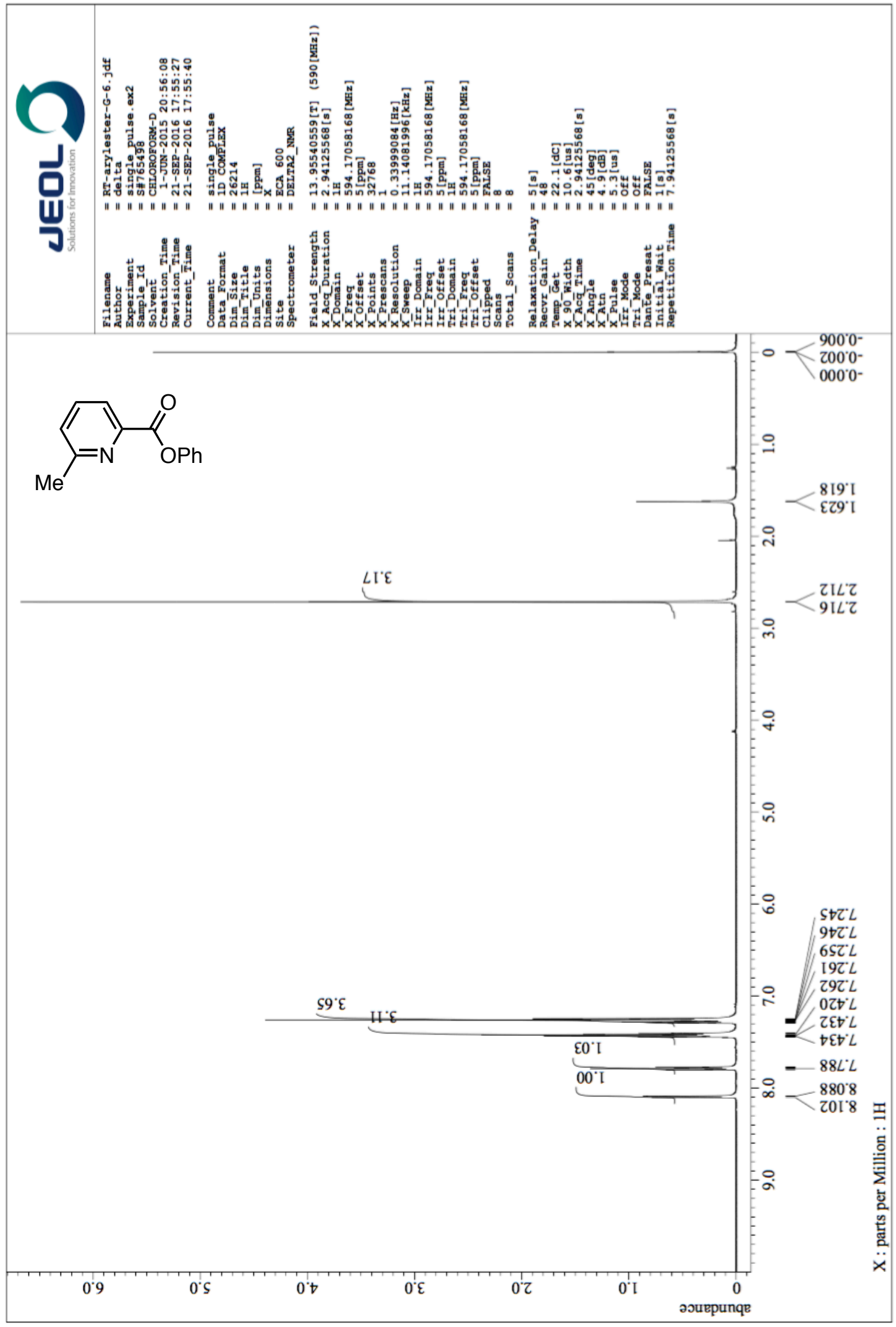


${ }^{13} \mathrm{C}$ NMR of $1 \mathrm{C}\left(100 \mathrm{MHz}, \mathrm{CDCl}_{3}\right)$

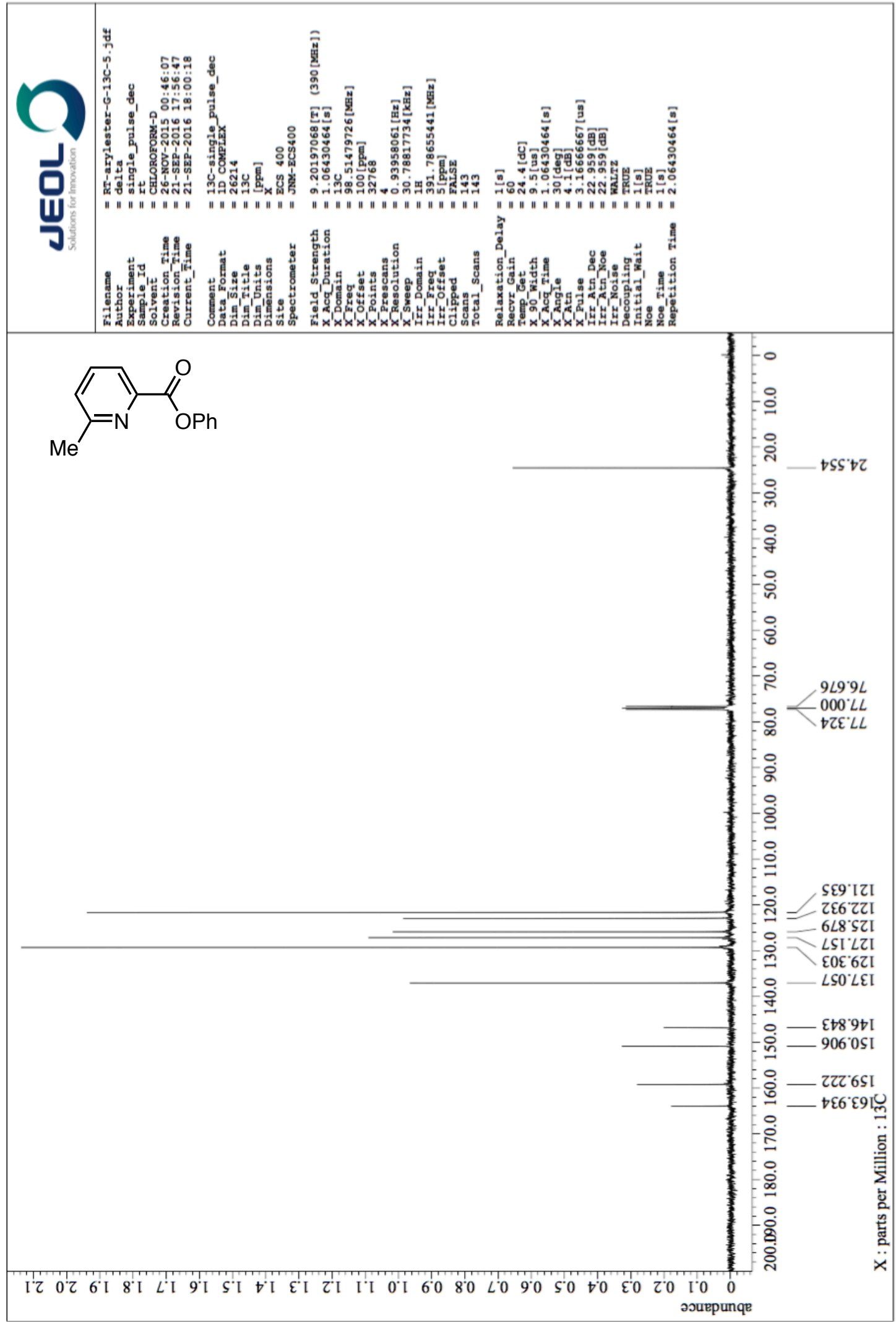


${ }^{1} \mathrm{H}$ NMR of $1 \mathbf{E}\left(400 \mathrm{MHz}, \mathrm{CDCl}_{3}\right)$

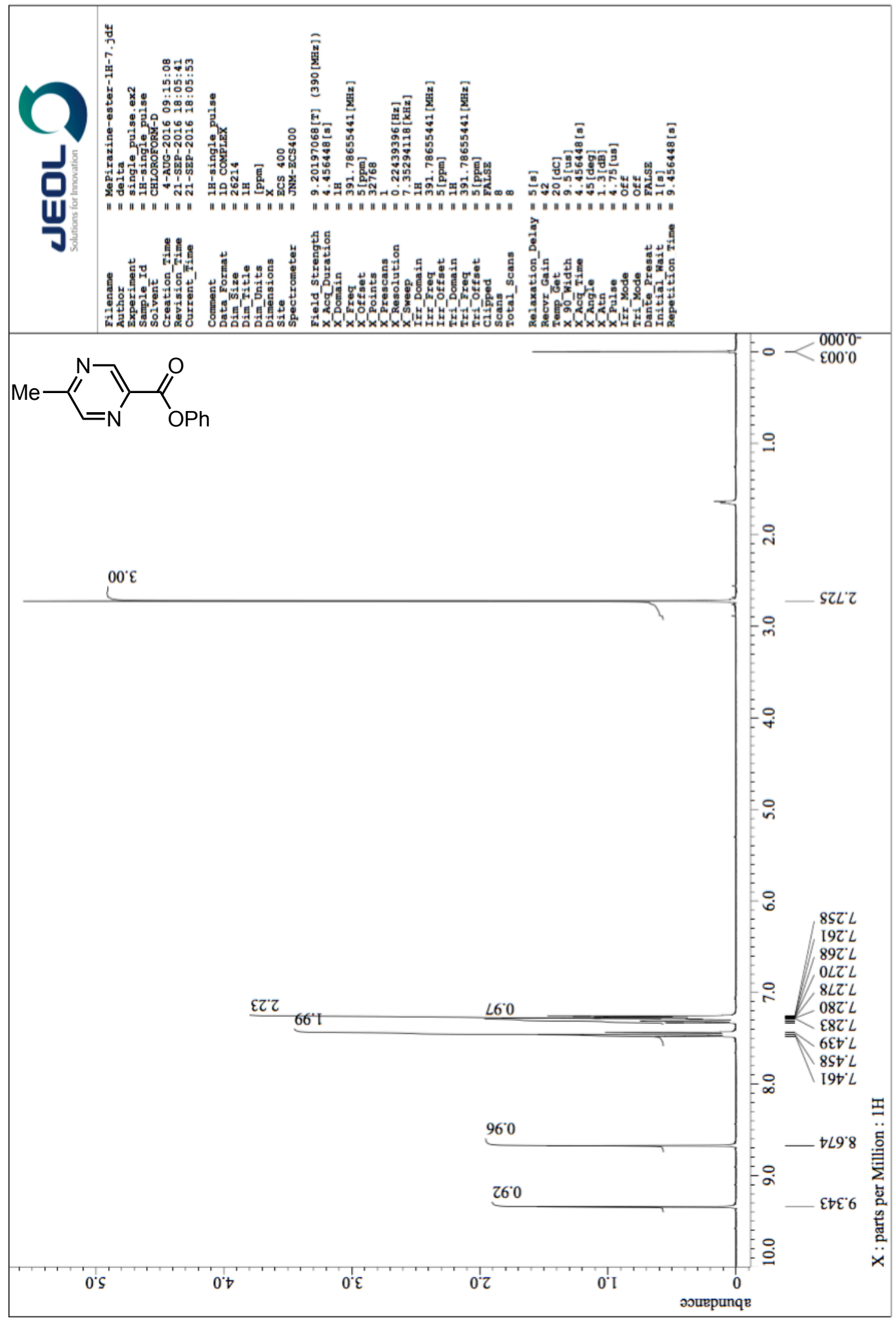


${ }^{13} \mathrm{C}$ NMR of $1 \mathbf{E}\left(100 \mathrm{MHz}, \mathrm{CDCl}_{3}\right)$

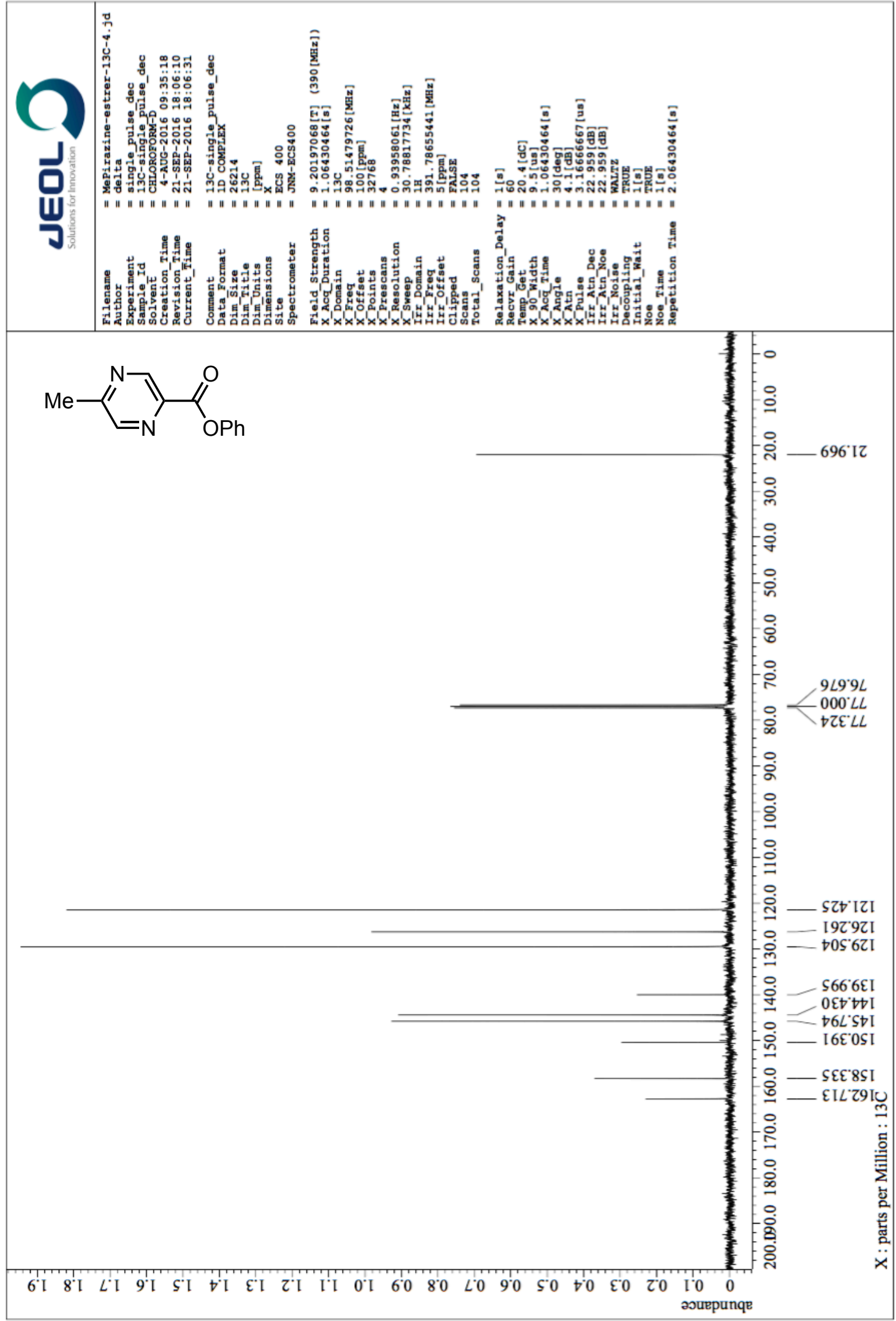


${ }^{1} \mathrm{H}$ NMR of $\mathbf{1 F}\left(400 \mathrm{MHz}, \mathrm{CDCl}_{3}\right)$

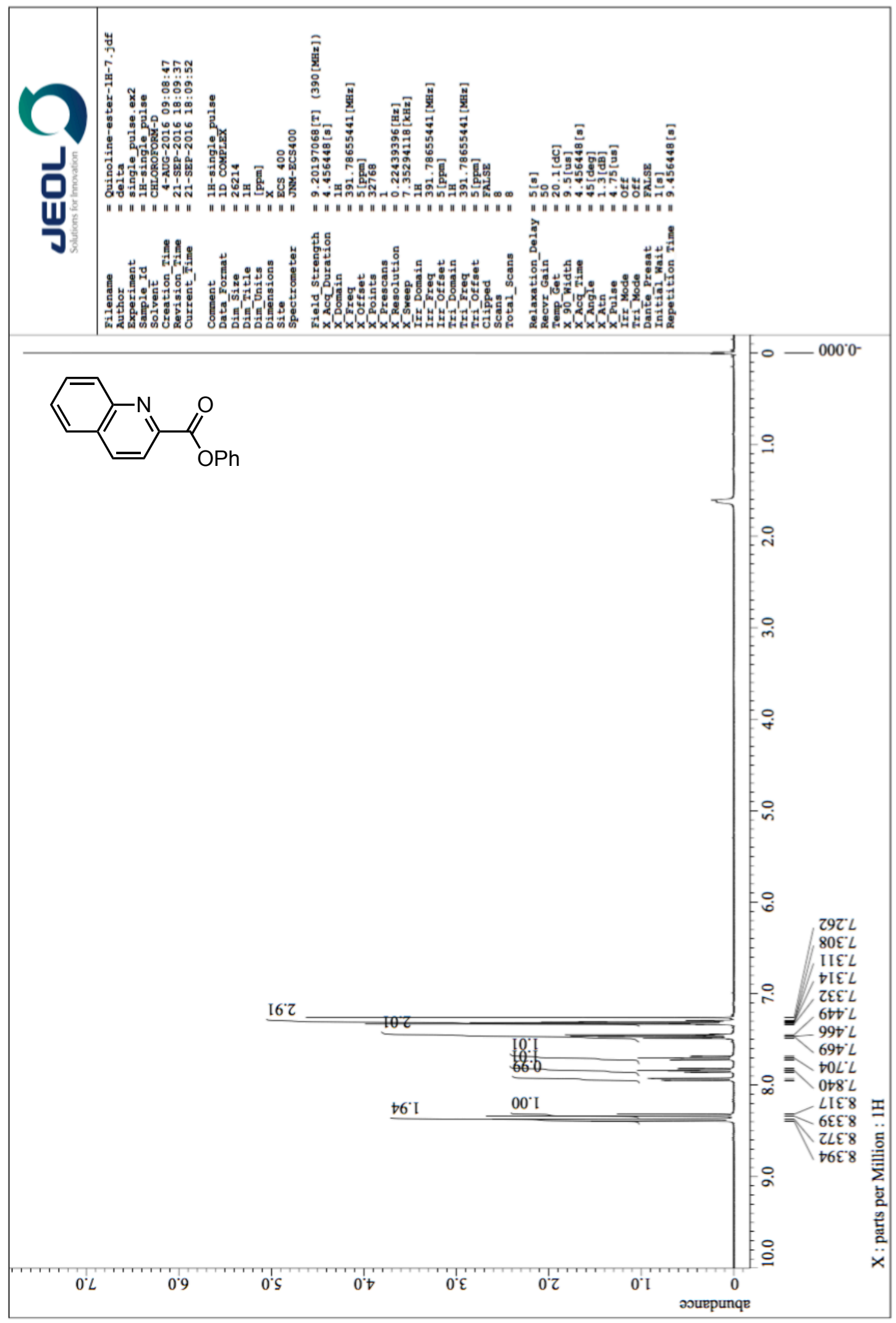


${ }^{13} \mathrm{C}$ NMR of $\mathbf{1 F}\left(100 \mathrm{MHz}, \mathrm{CDCl}_{3}\right)$

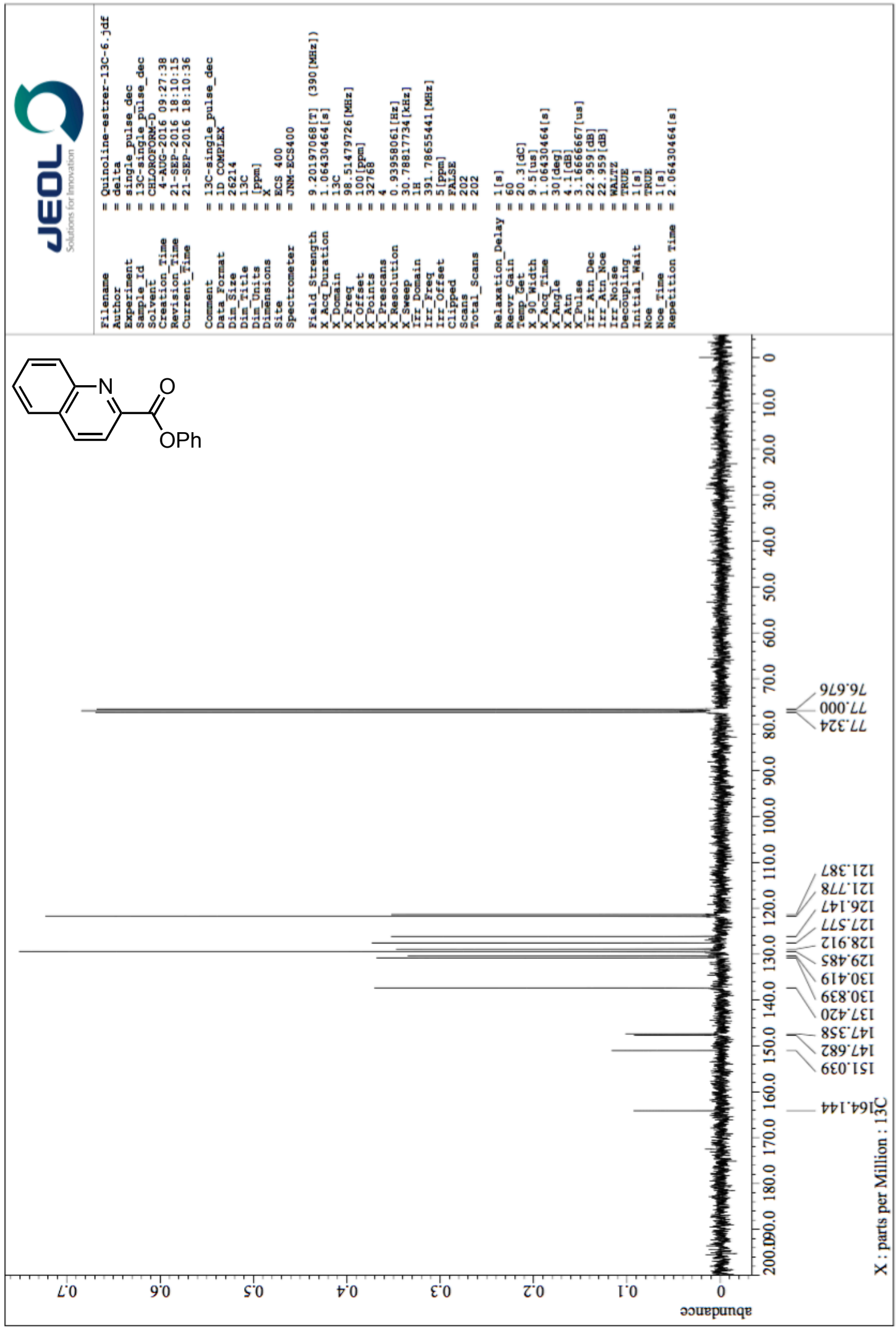


${ }^{1} \mathrm{H}$ NMR of $\mathbf{1 G}\left(400 \mathrm{MHz}, \mathrm{CDCl}_{3}\right)$

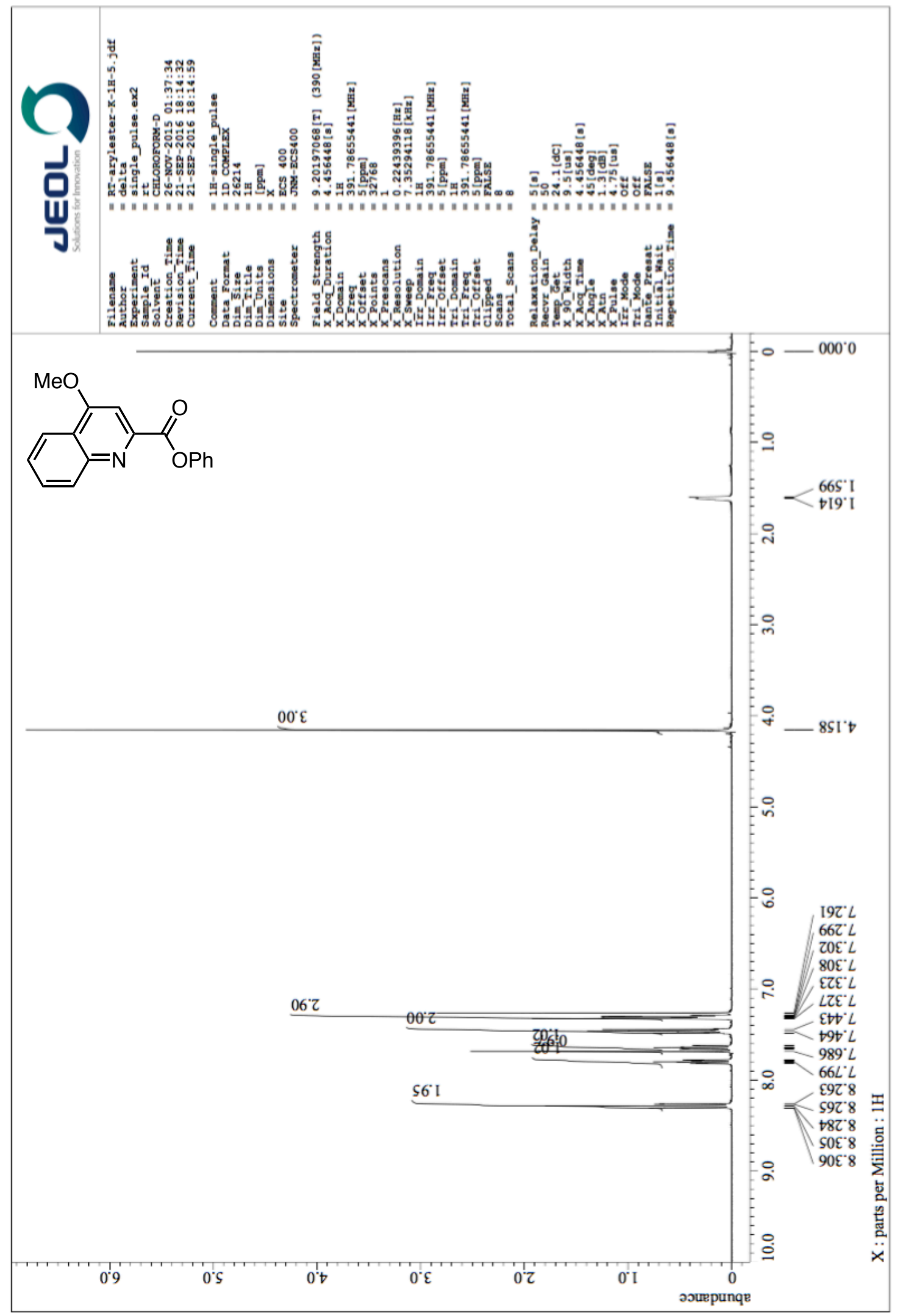


${ }^{13} \mathrm{C}$ NMR of $1 G\left(100 \mathrm{MHz}, \mathrm{CDCl}_{3}\right)$

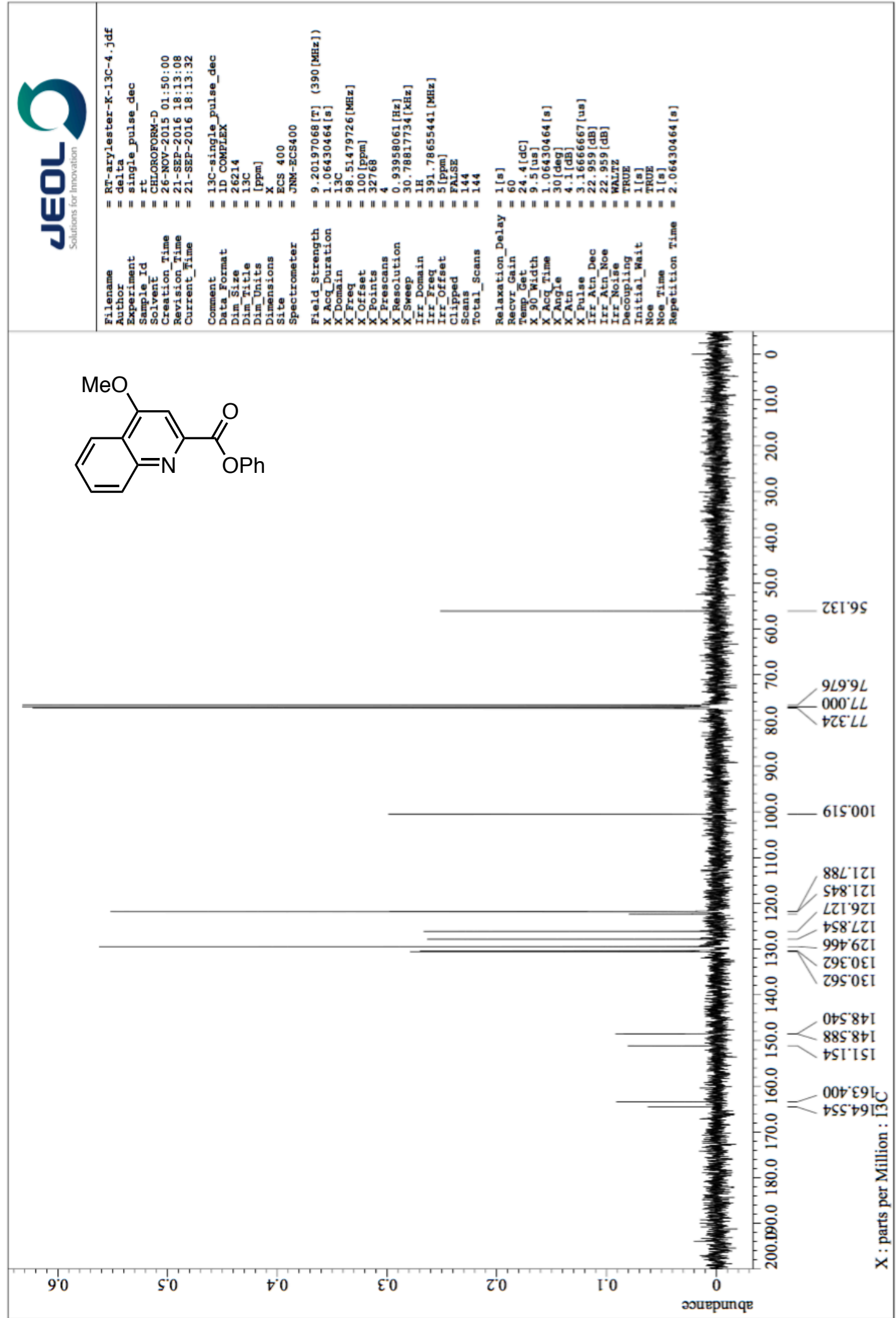


${ }^{1} \mathrm{H}$ NMR of $\mathbf{1 H}\left(400 \mathrm{MHz}, \mathrm{CDCl}_{3}\right)$

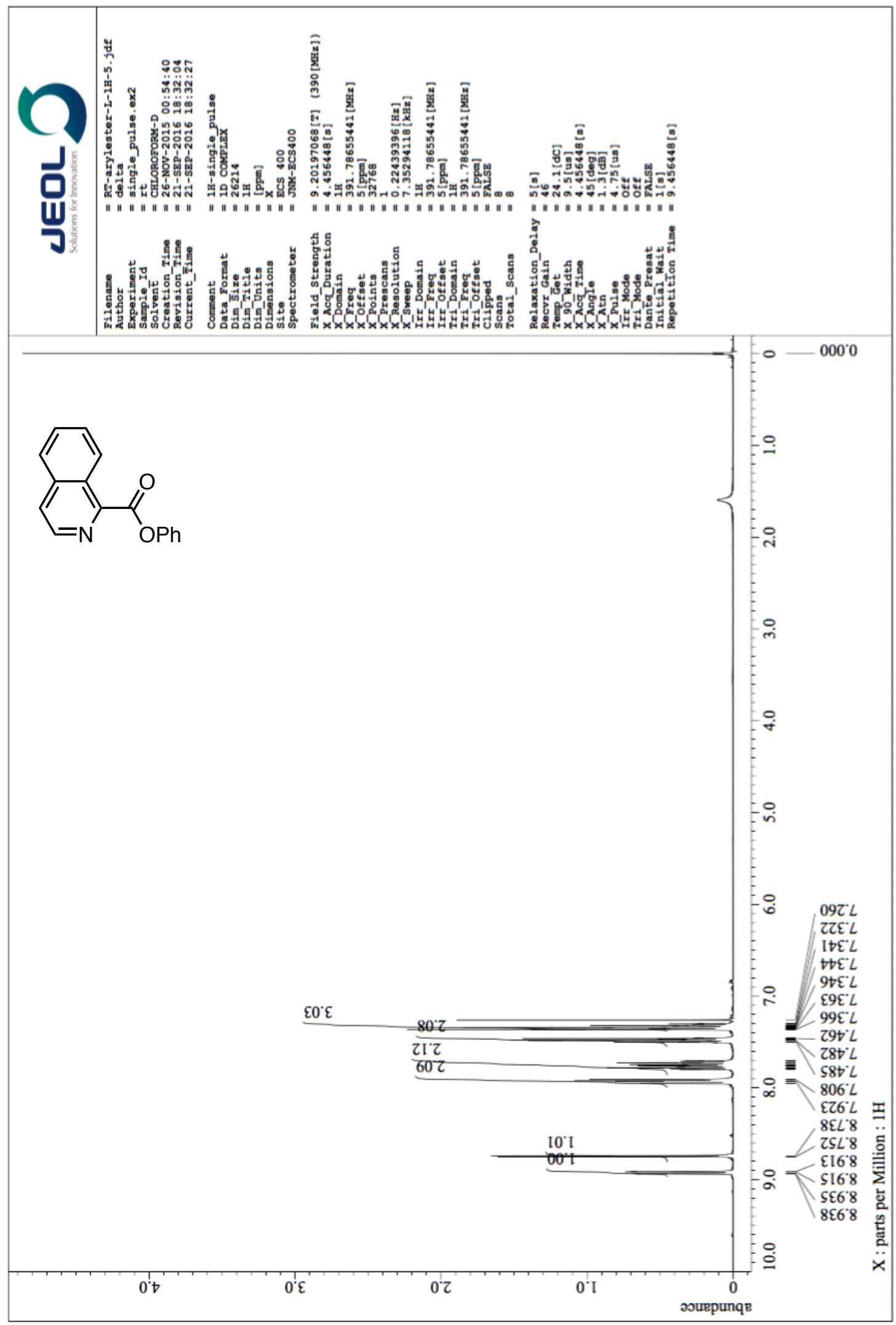


${ }^{13} \mathrm{C}$ NMR of $\mathbf{1 H}\left(100 \mathrm{MHz}, \mathrm{CDCl}_{3}\right)$

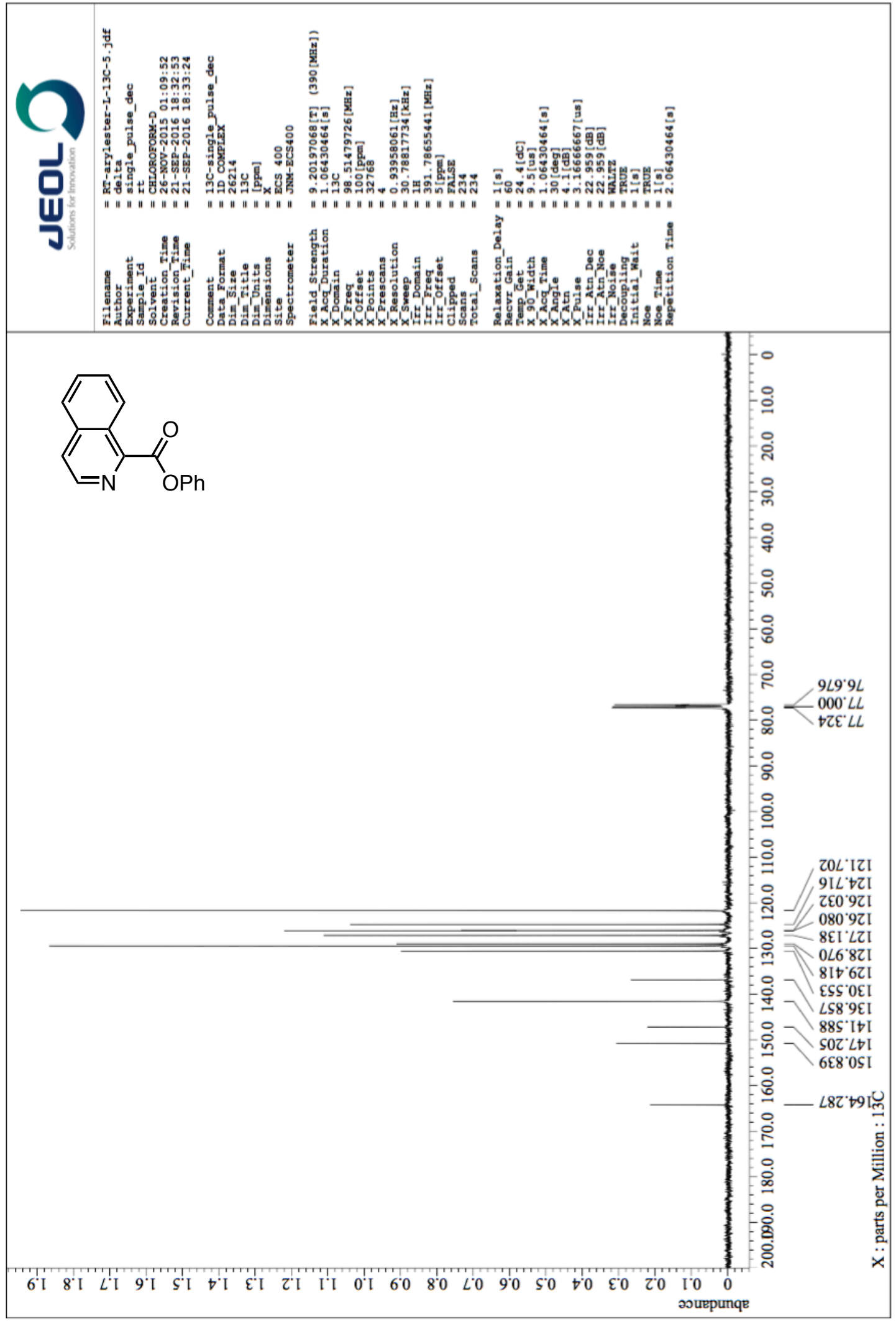


${ }^{1} \mathrm{H}$ NMR of $\mathbf{3 A a}\left(400 \mathrm{MHz}, \mathrm{CDCl}_{3}\right)$

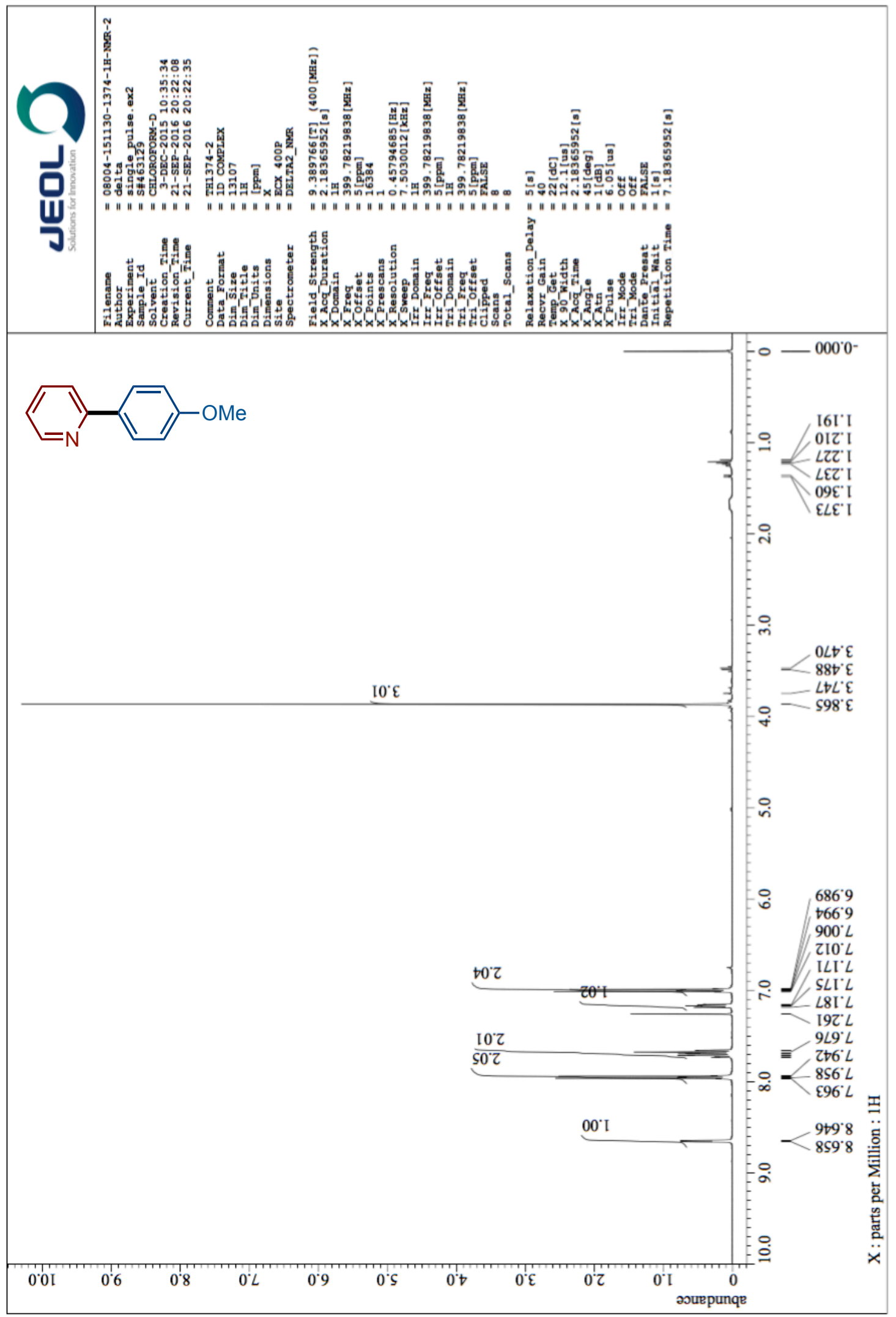


${ }^{13} \mathrm{C}$ NMR of $\mathbf{3 A a}\left(100 \mathrm{MHz}, \mathrm{CDCl}_{3}\right)$

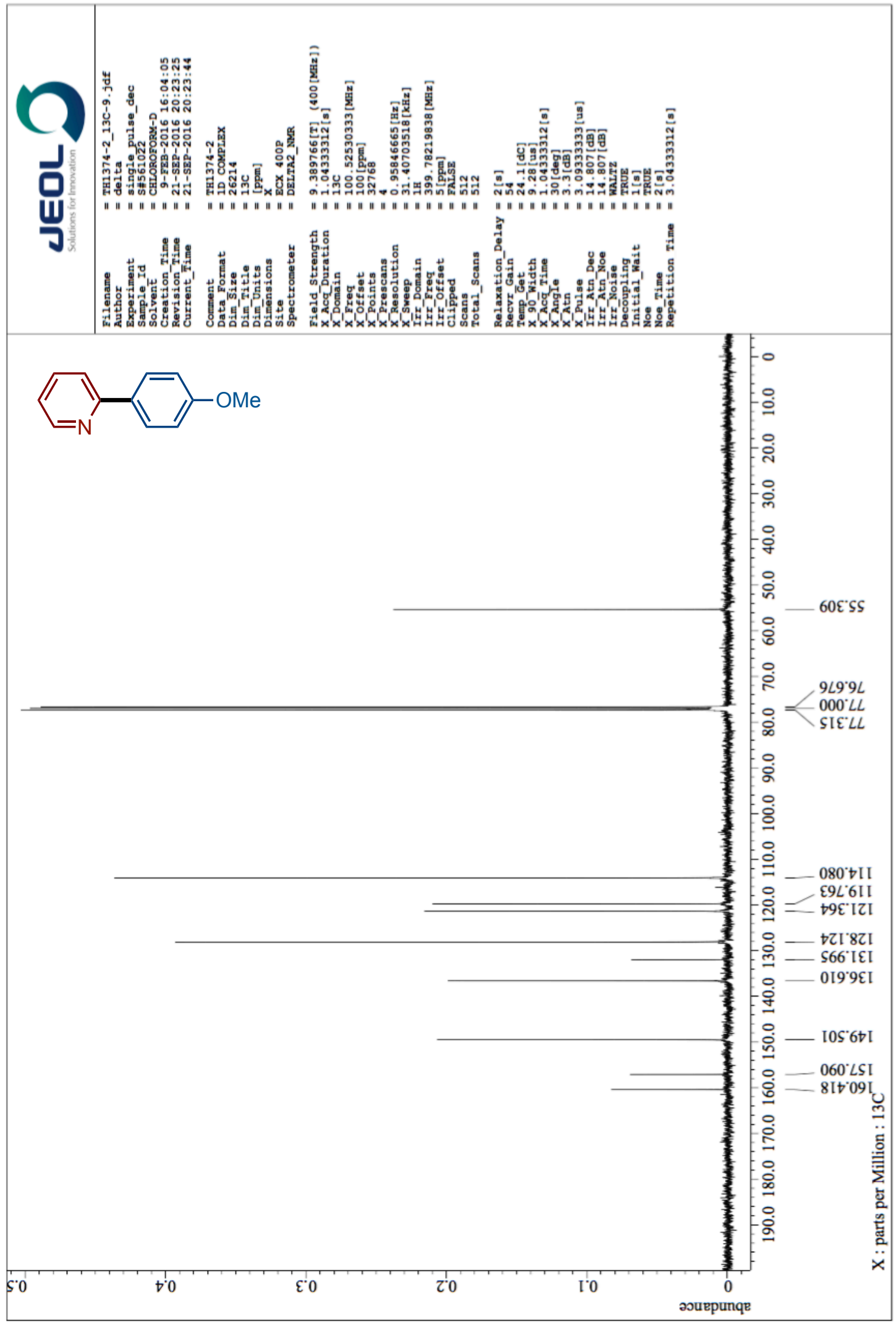


${ }^{1} \mathrm{H}$ NMR 3Ba $\left(400 \mathrm{MHz}, \mathrm{CDCl}_{3}\right)$

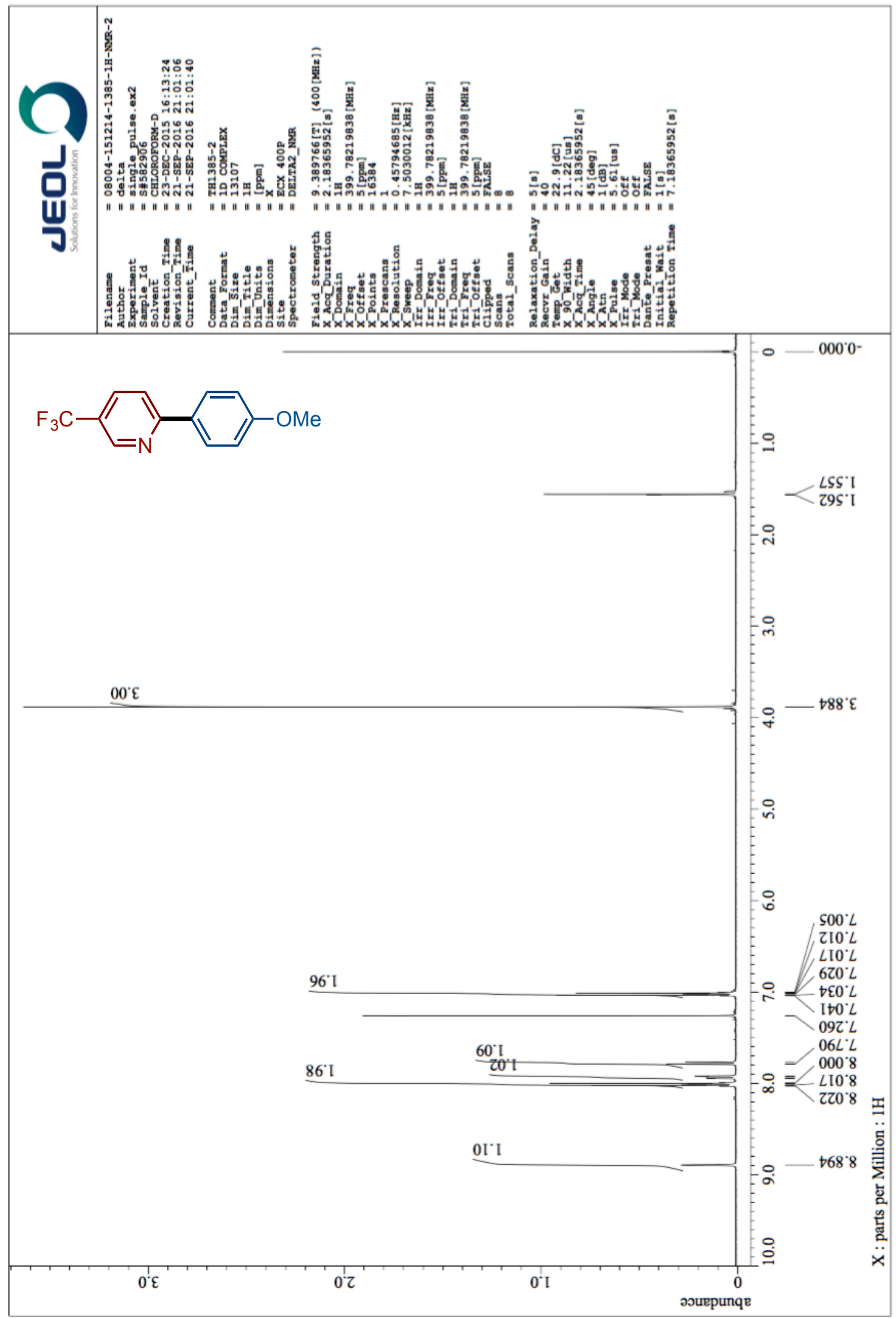


${ }^{13} \mathrm{C}$ NMR 3Ba $\left(100 \mathrm{MHz}, \mathrm{CDCl}_{3}\right)$

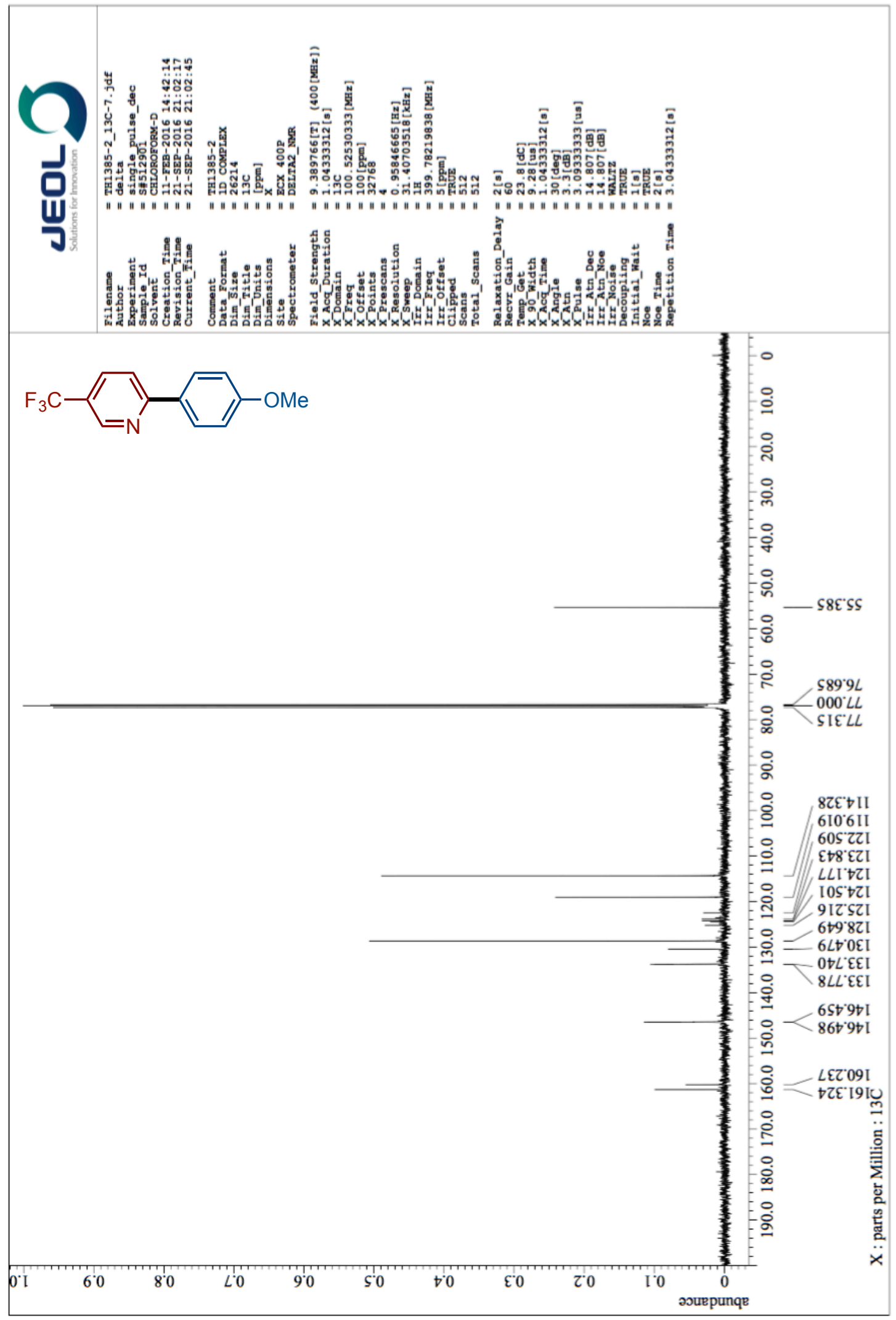


${ }^{1} \mathrm{H}$ NMR 3Ca $\left(400 \mathrm{MHz}, \mathrm{CDCl}_{3}\right)$

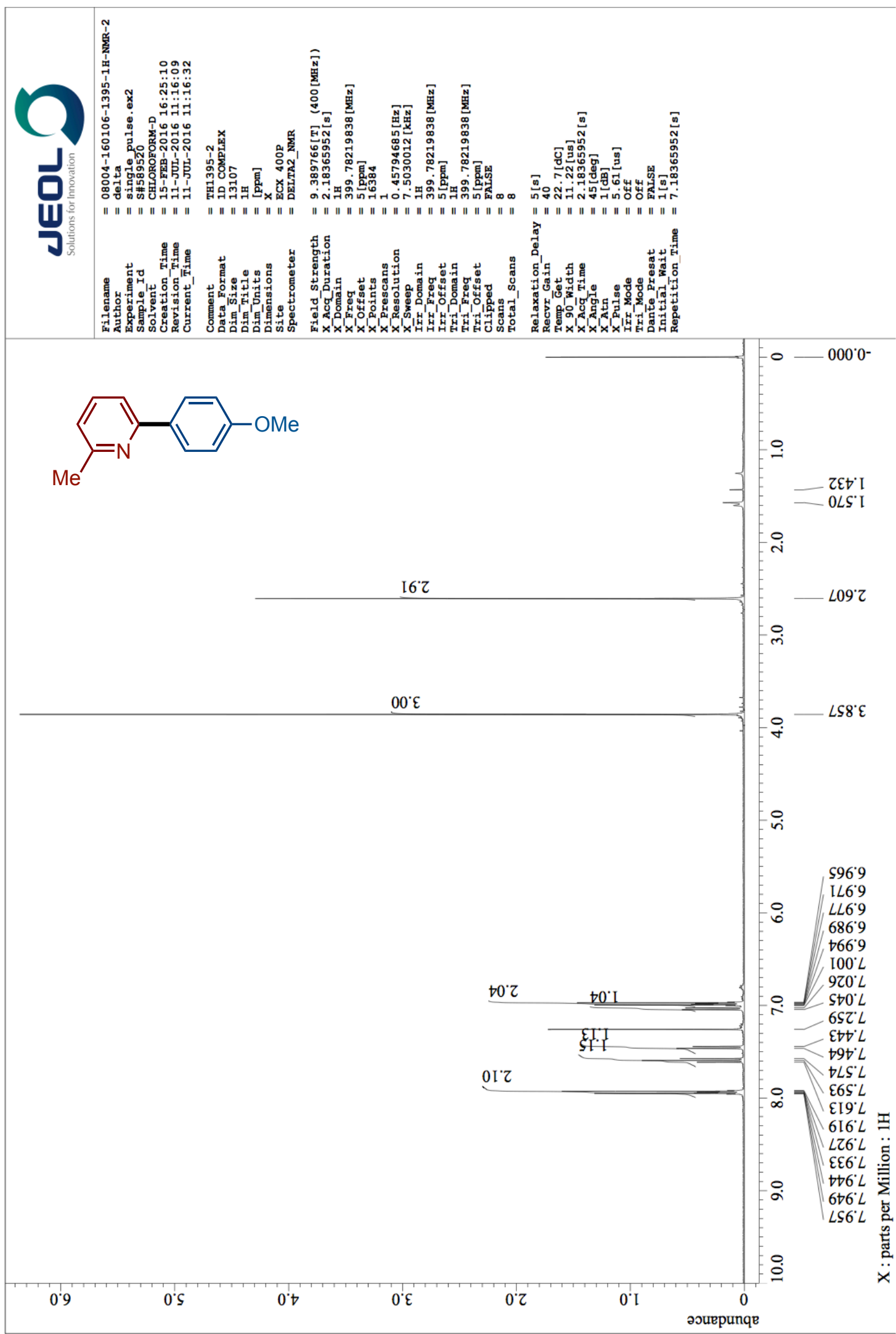


${ }^{13} \mathrm{C}$ NMR 3Ca $\left(100 \mathrm{MHz}, \mathrm{CDCl}_{3}\right)$

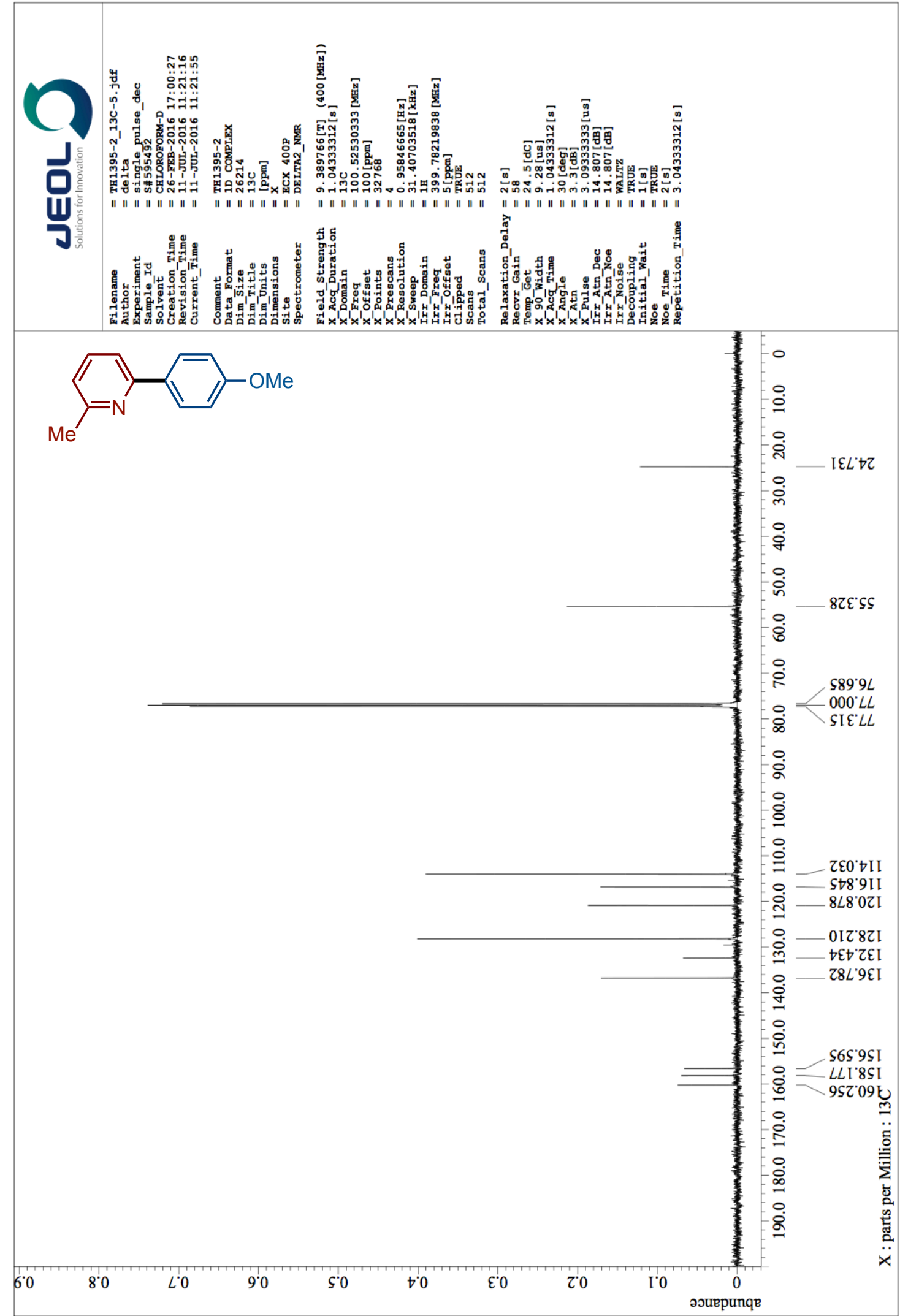


${ }^{1} \mathrm{H}$ NMR 3Da $\left(400 \mathrm{MHz}, \mathrm{CDCl}_{3}\right)$

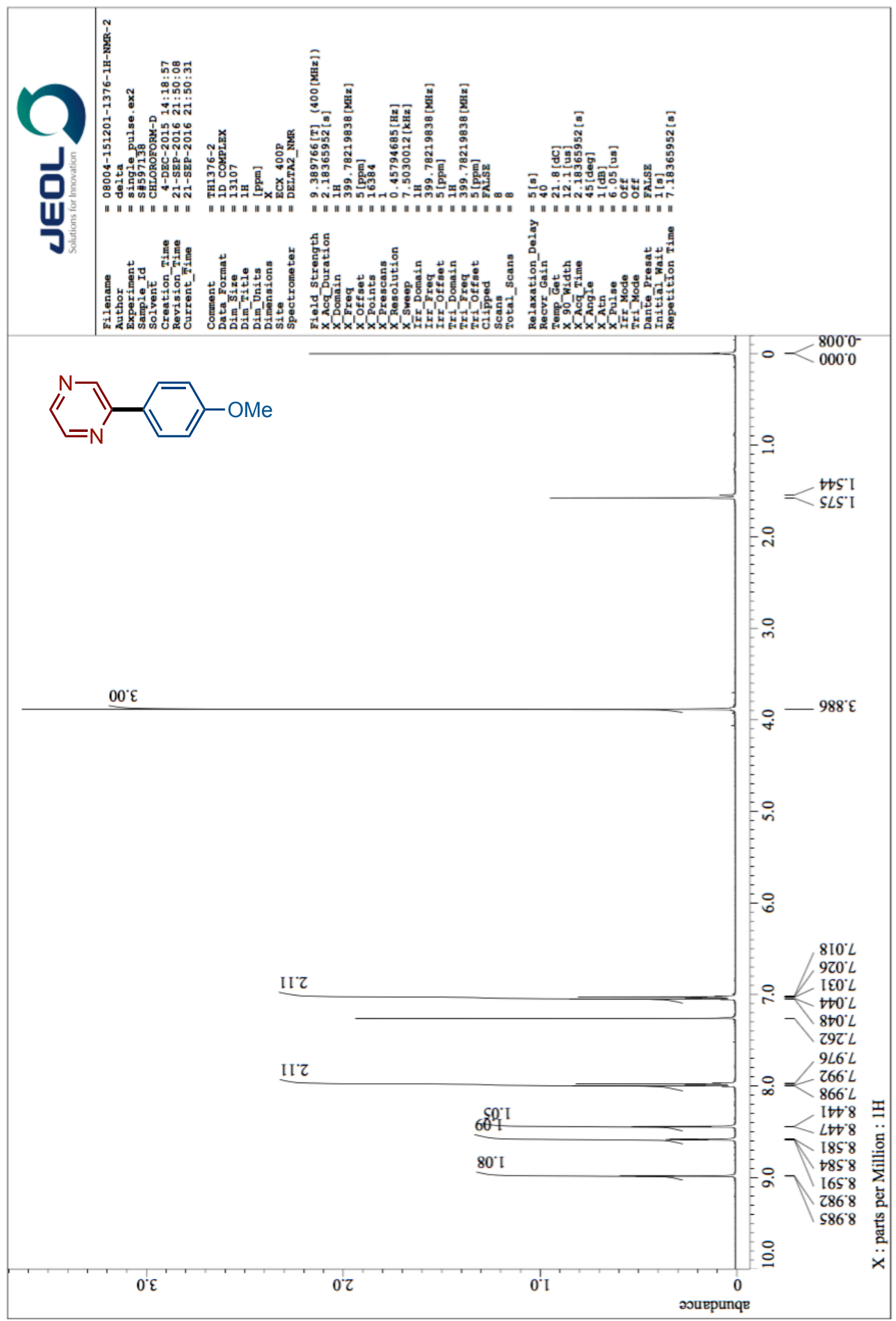


${ }^{13} \mathrm{C}$ NMR 3Da $\left(100 \mathrm{MHz}, \mathrm{CDCl}_{3}\right)$

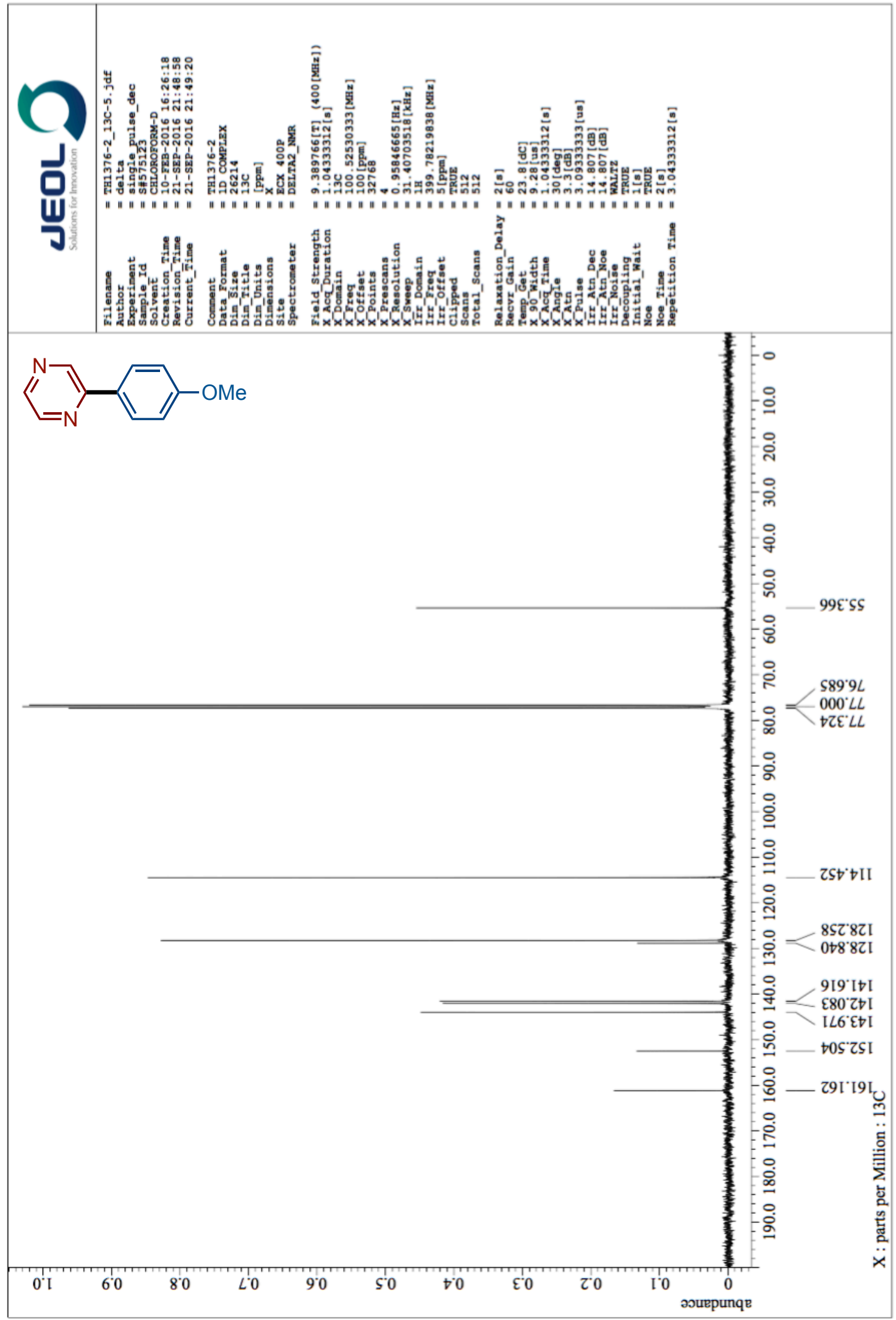


${ }^{1} \mathrm{H}$ NMR 3Ea $\left(400 \mathrm{MHz}, \mathrm{CDCl}_{3}\right)$

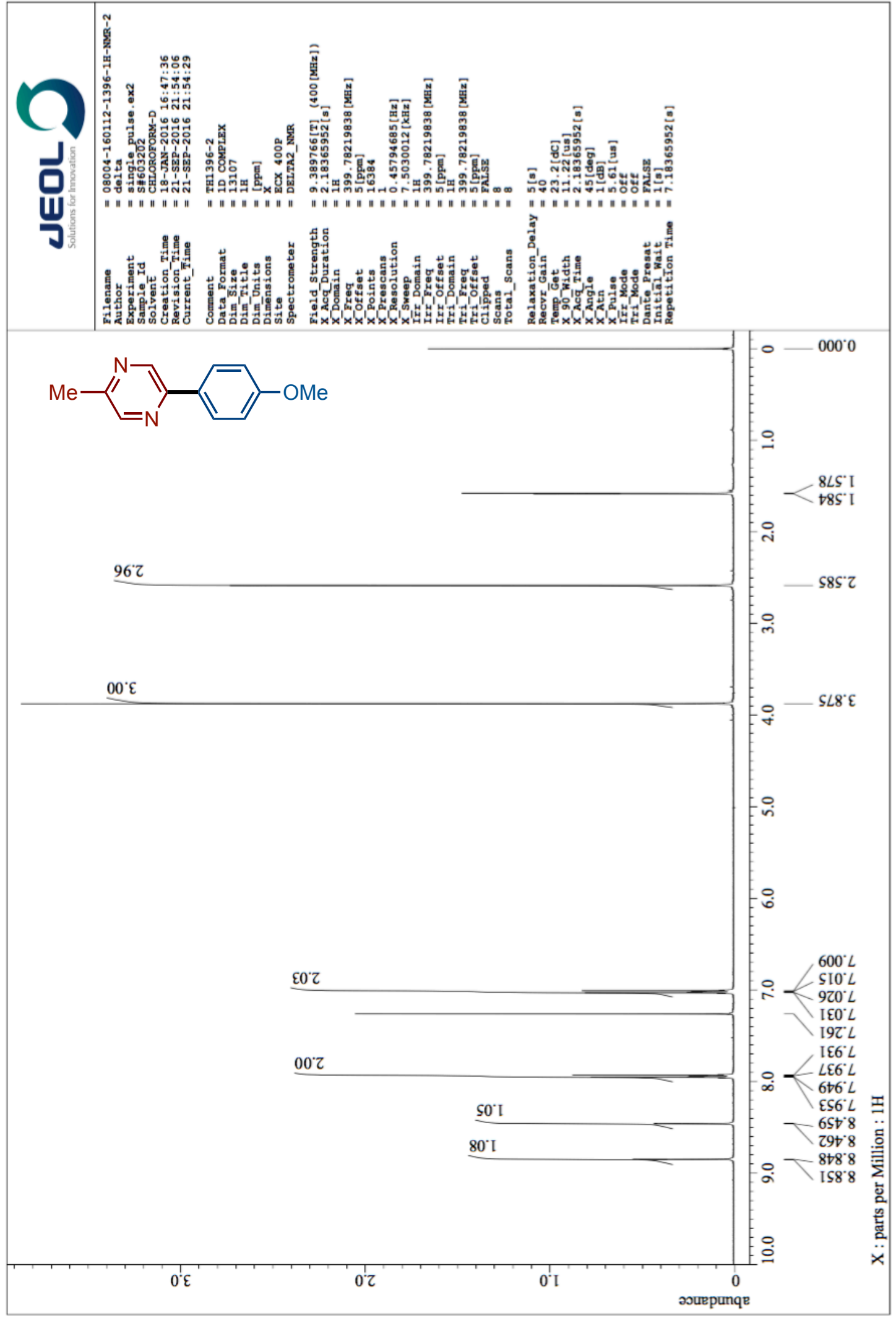


${ }^{13} \mathrm{C}$ NMR 3Ea $\left(100 \mathrm{MHz}, \mathrm{CDCl}_{3}\right)$

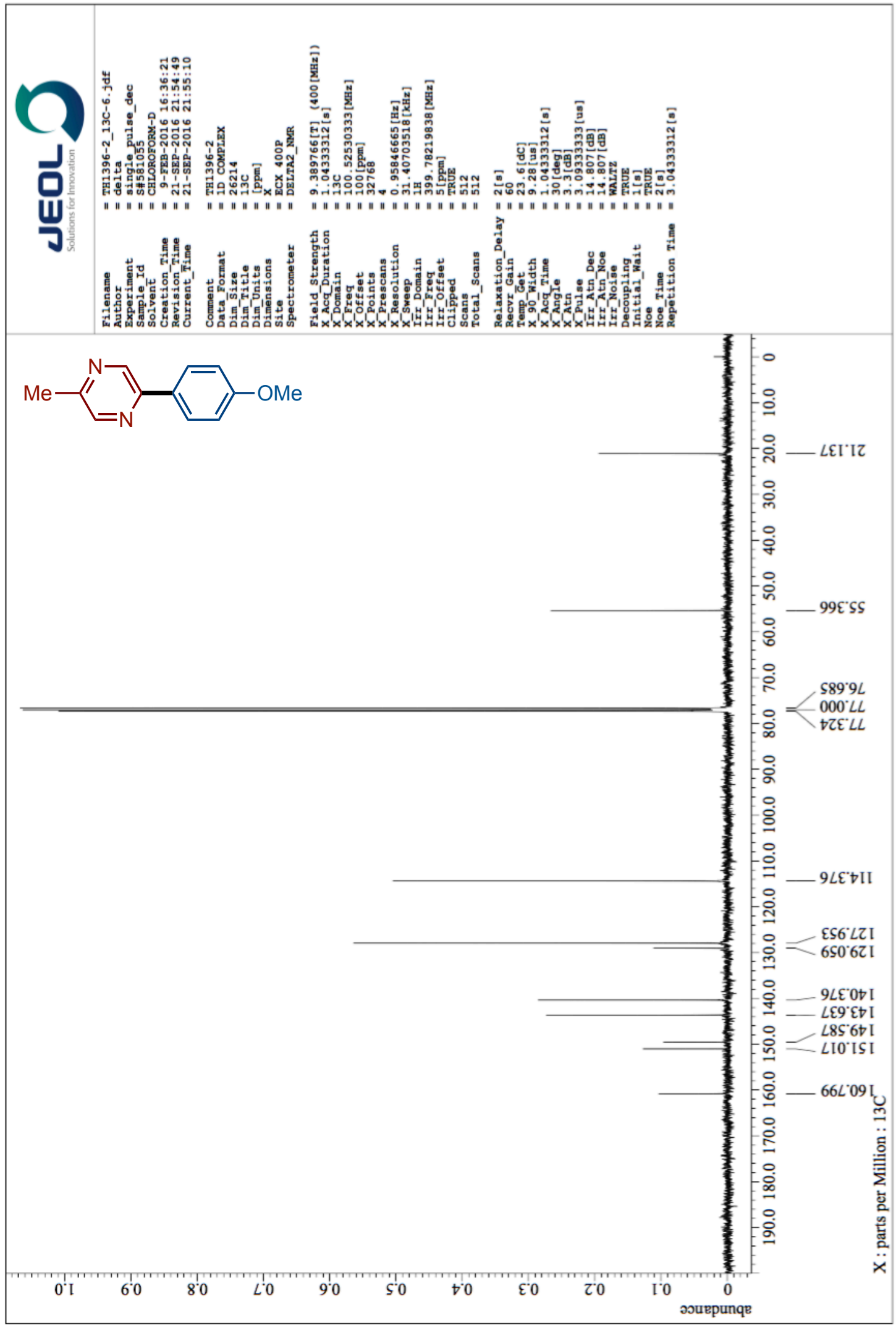


${ }^{1} \mathrm{H}$ NMR 3Fa $\left(400 \mathrm{MHz}, \mathrm{CDCl}_{3}\right)$

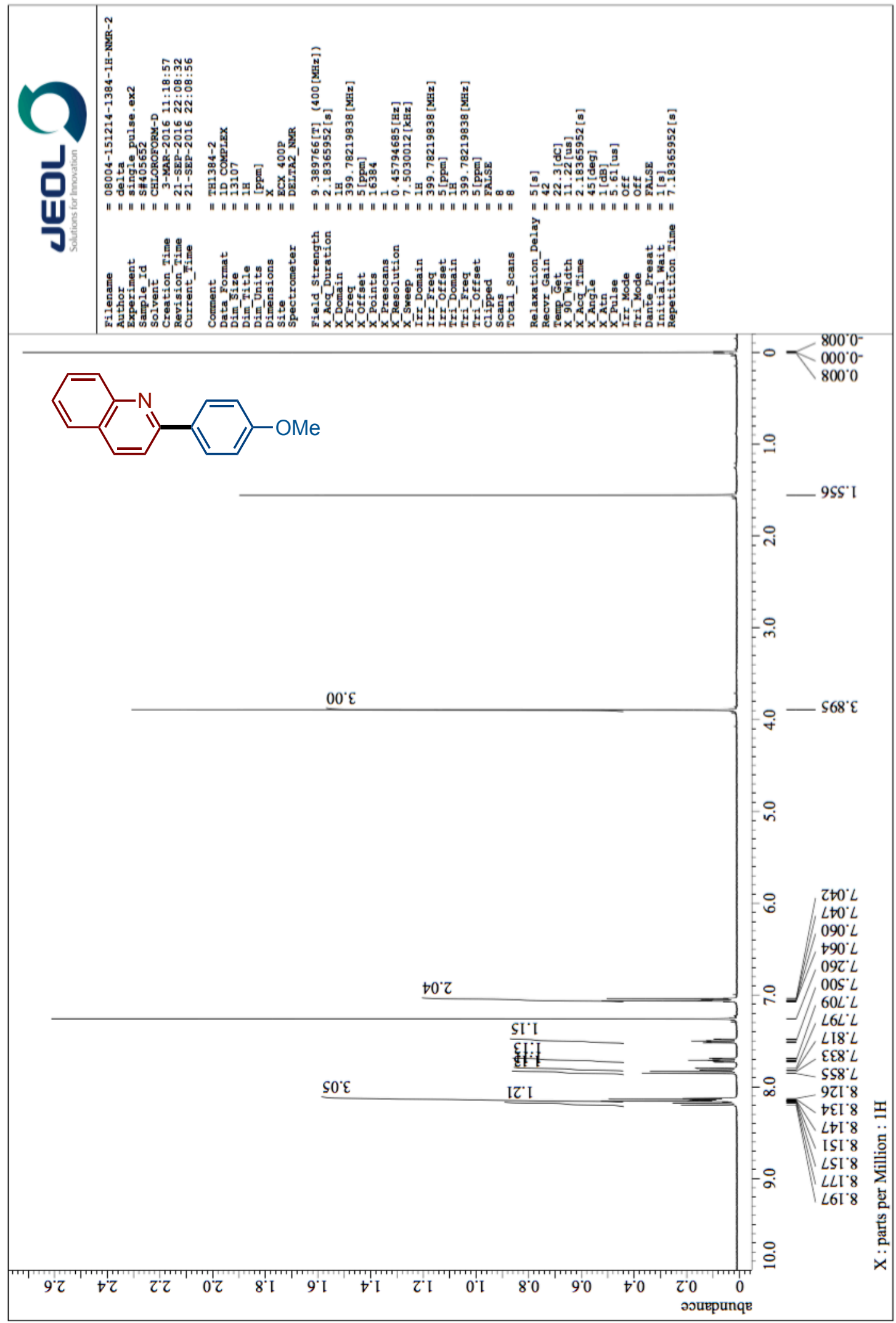


${ }^{13} \mathrm{C}$ NMR 3Fa $\left(100 \mathrm{MHz}, \mathrm{CDCl}_{3}\right)$

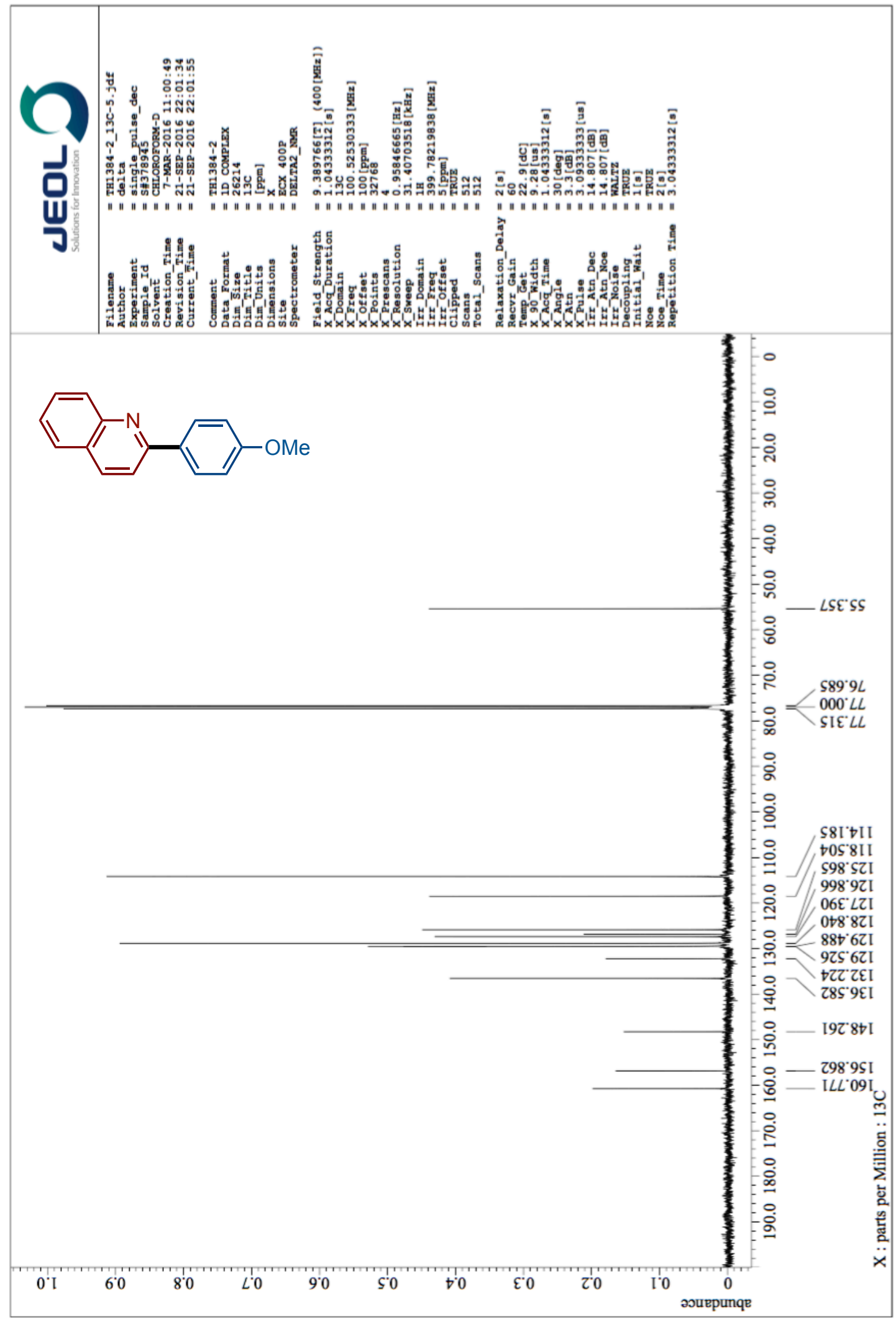


${ }^{1} \mathrm{H}$ NMR 3Ga $\left(400 \mathrm{MHz}, \mathrm{CDCl}_{3}\right)$

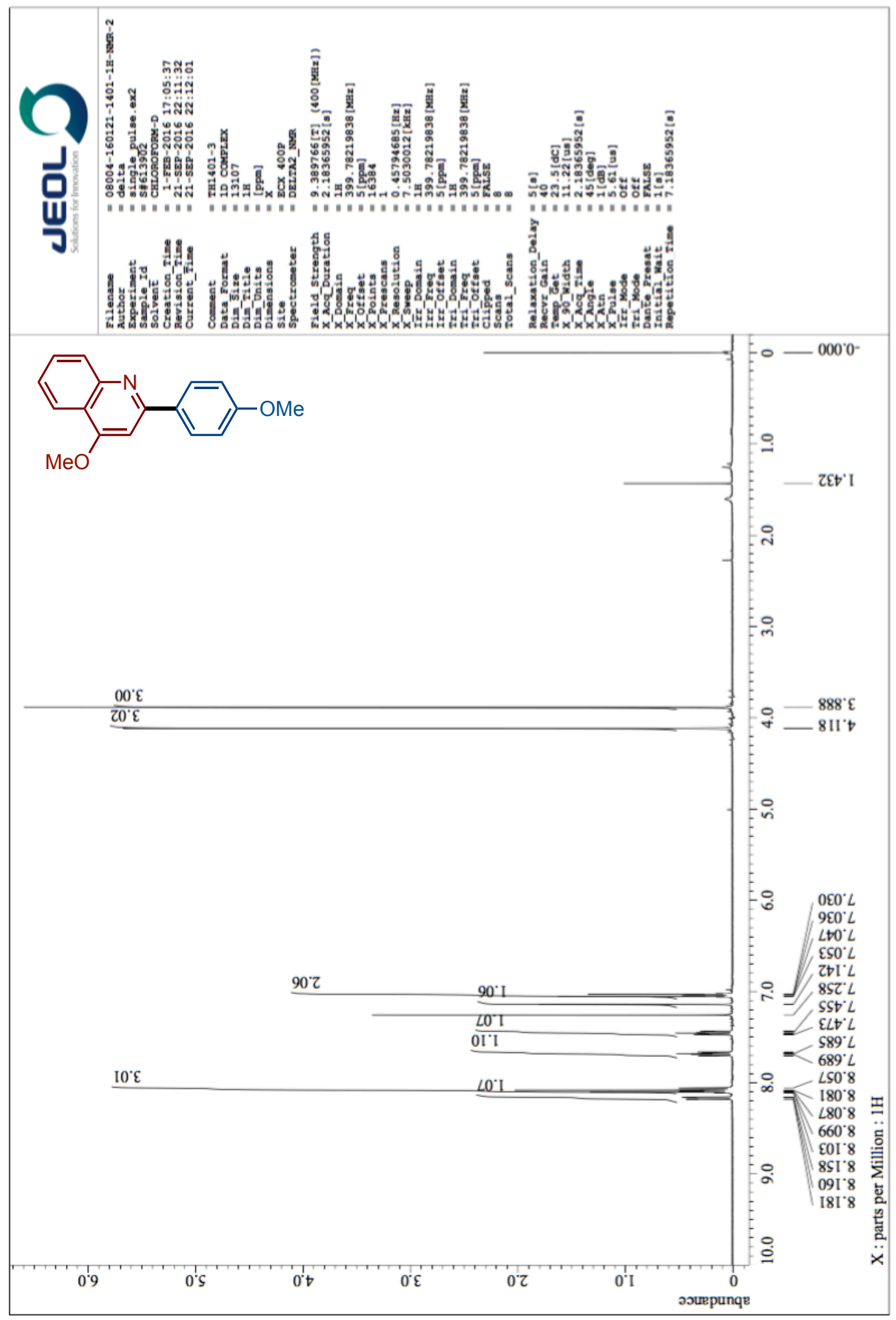


${ }^{13} \mathrm{C}$ NMR 3Ga $\left(100 \mathrm{MHz}, \mathrm{CDCl}_{3}\right)$

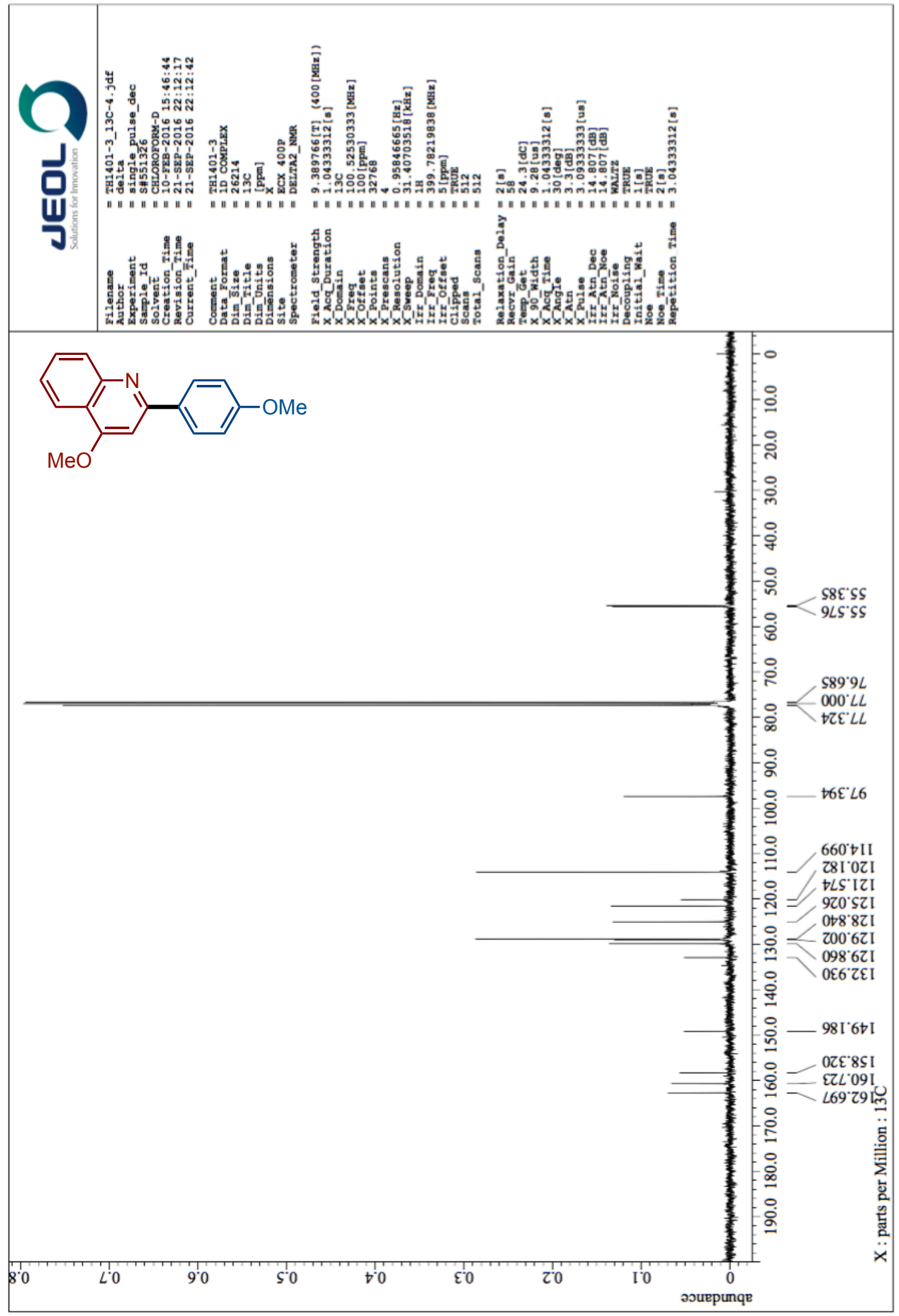


${ }^{1} \mathrm{H}$ NMR 3Ha $\left(400 \mathrm{MHz}, \mathrm{CDCl}_{3}\right)$

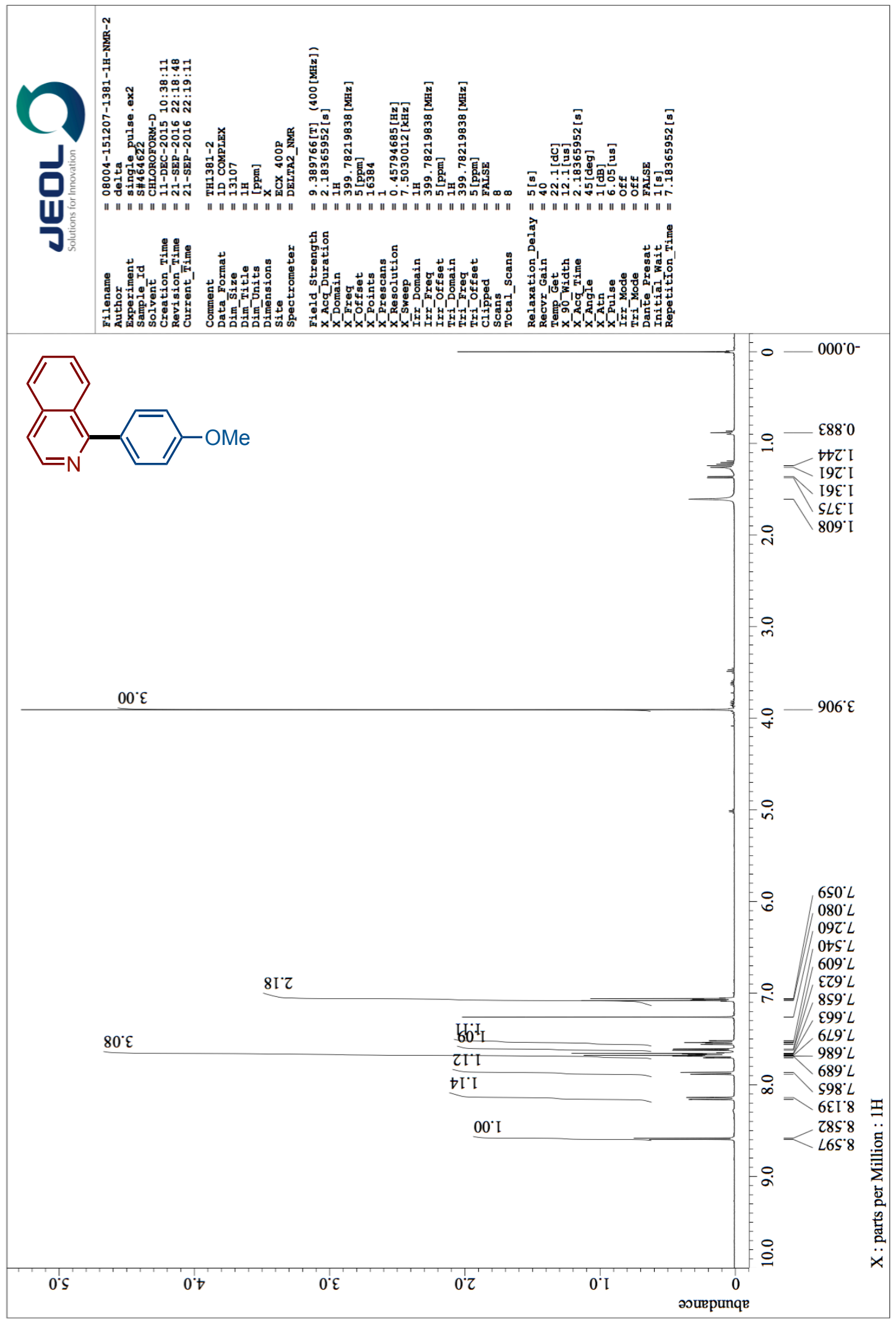


${ }^{13} \mathrm{C}$ NMR 3Ha $\left(100 \mathrm{MHz}, \mathrm{CDCl}_{3}\right)$

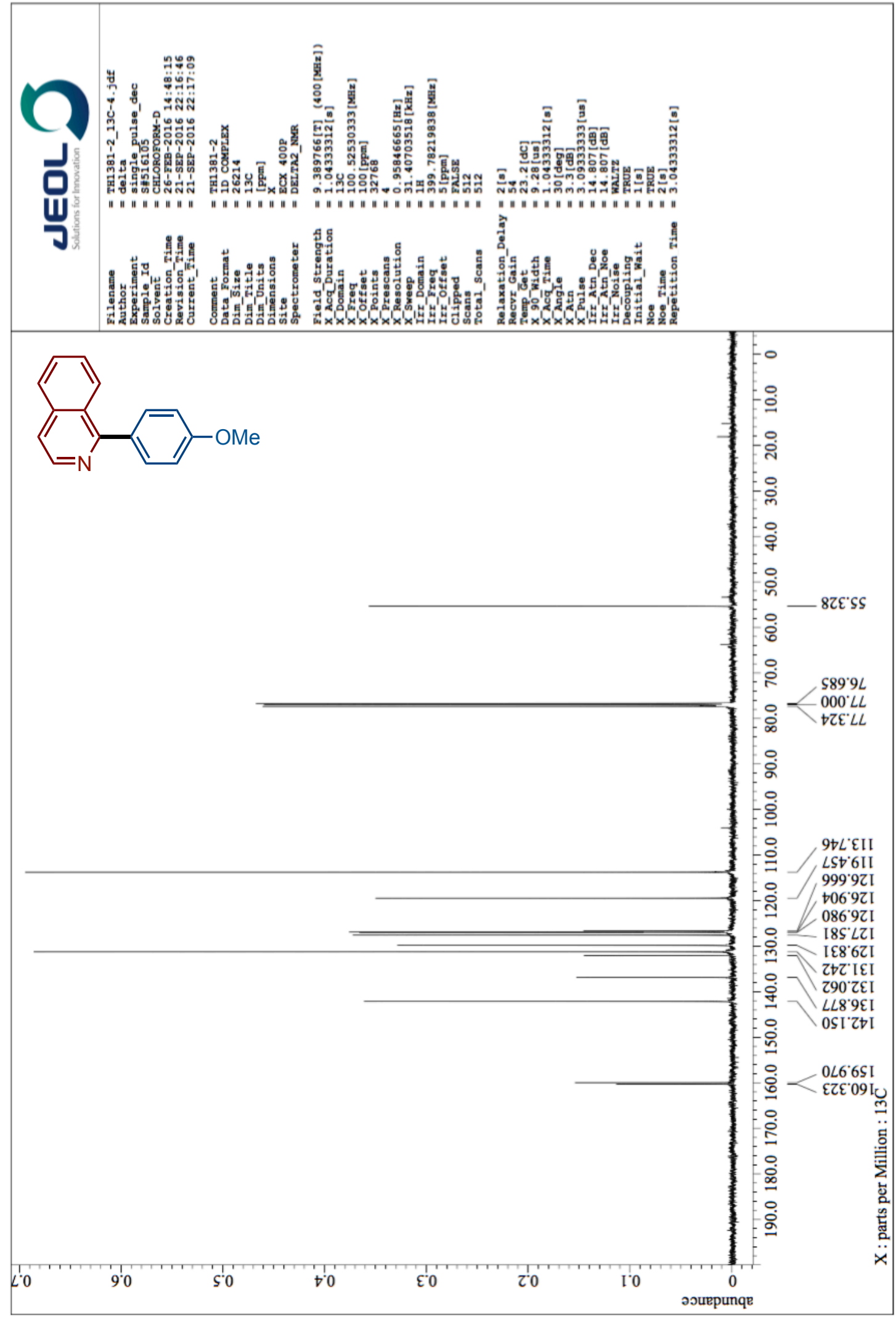


${ }^{1} \mathrm{H}$ NMR 3Ab $\left(400 \mathrm{MHz}, \mathrm{CDCl}_{3}\right)$

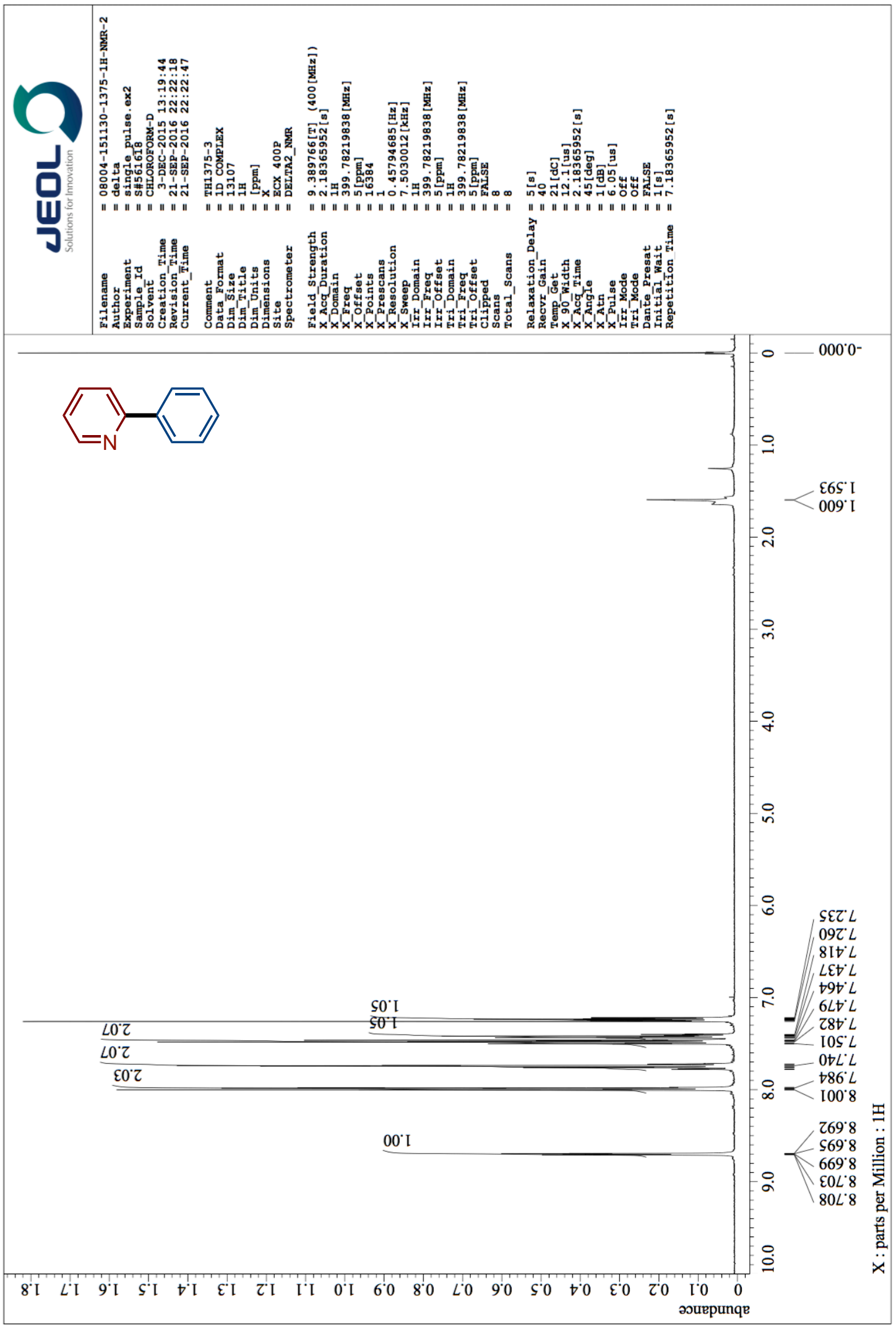


${ }^{13} \mathrm{C}$ NMR 3Ab $\left(100 \mathrm{MHz}, \mathrm{CDCl}_{3}\right)$

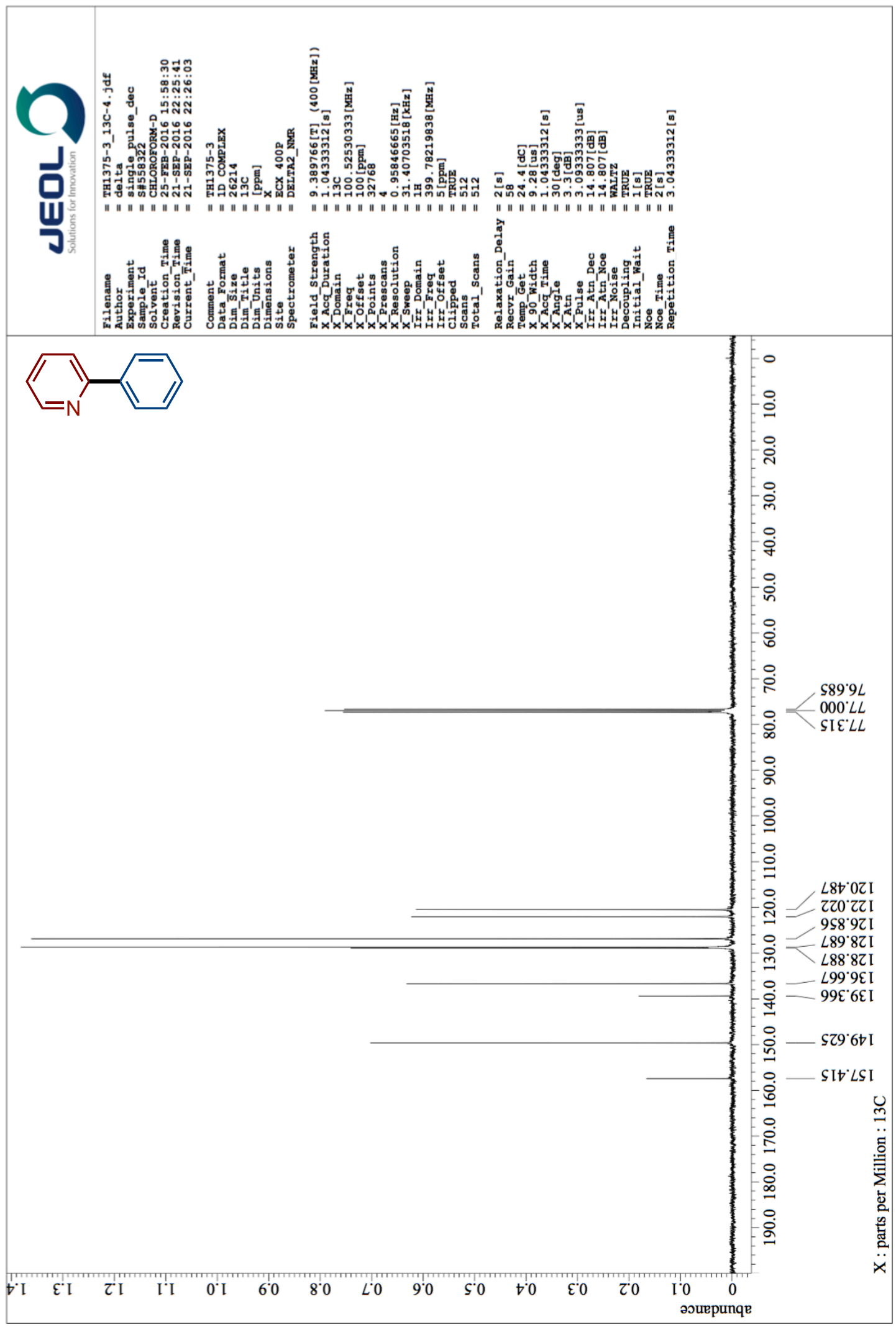


${ }^{1} \mathrm{H}$ NMR 3Ac $\left(400 \mathrm{MHz}, \mathrm{CDCl}_{3}\right)$

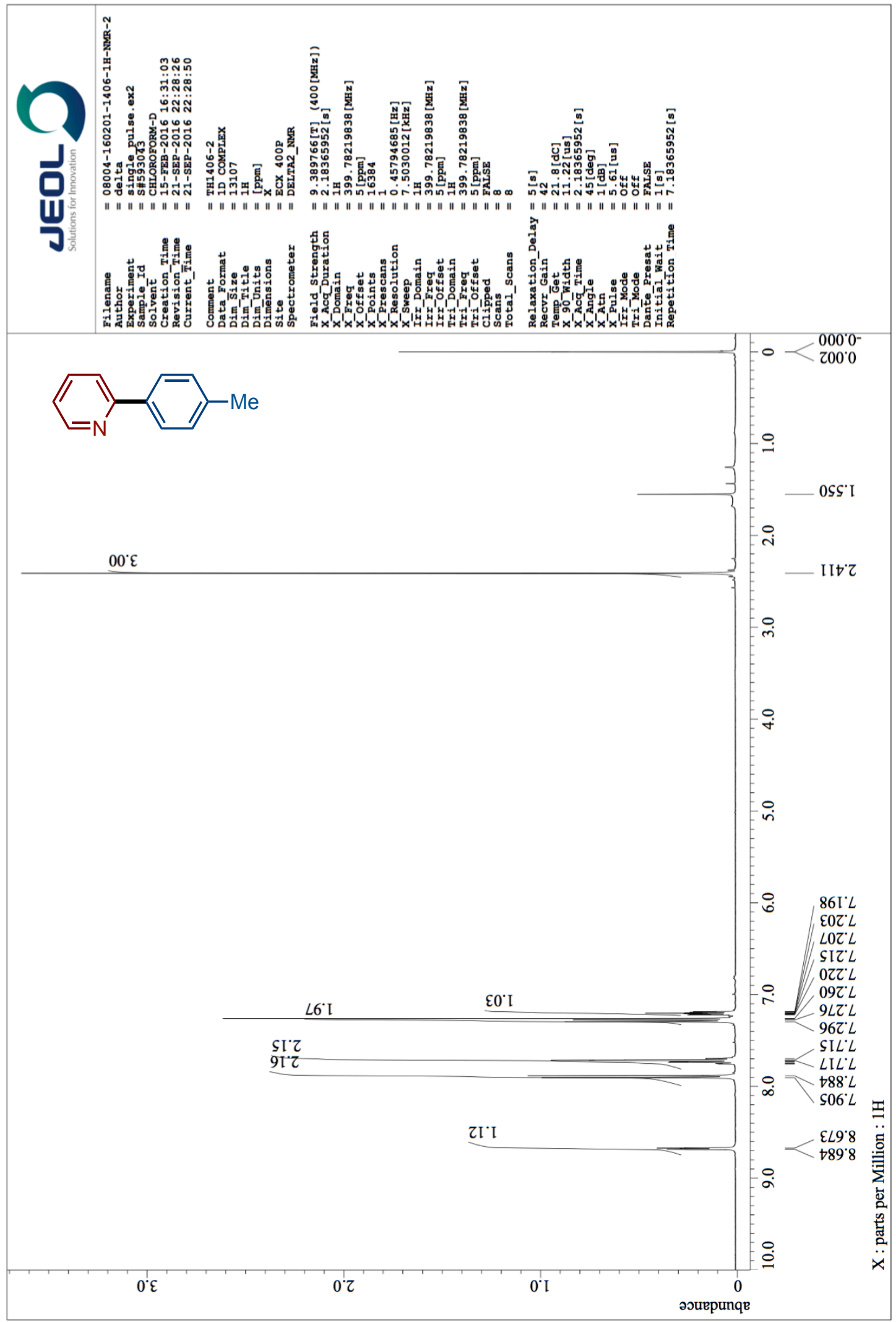


${ }^{13} \mathrm{C}$ NMR 3Ac $\left(100 \mathrm{MHz}, \mathrm{CDCl}_{3}\right)$

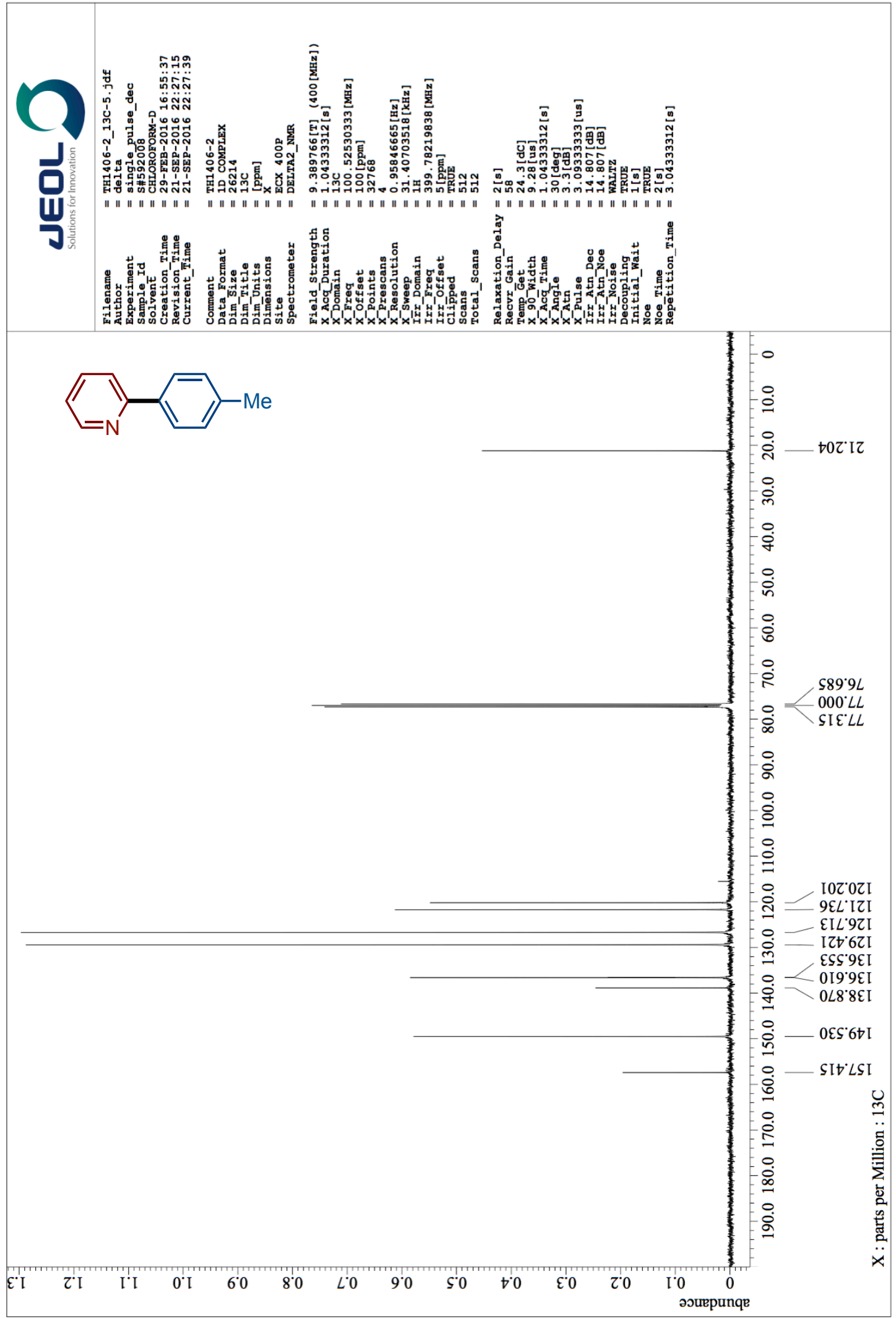


${ }^{1} \mathrm{H}$ NMR 3Ad $\left(400 \mathrm{MHz}, \mathrm{CDCl}_{3}\right)$

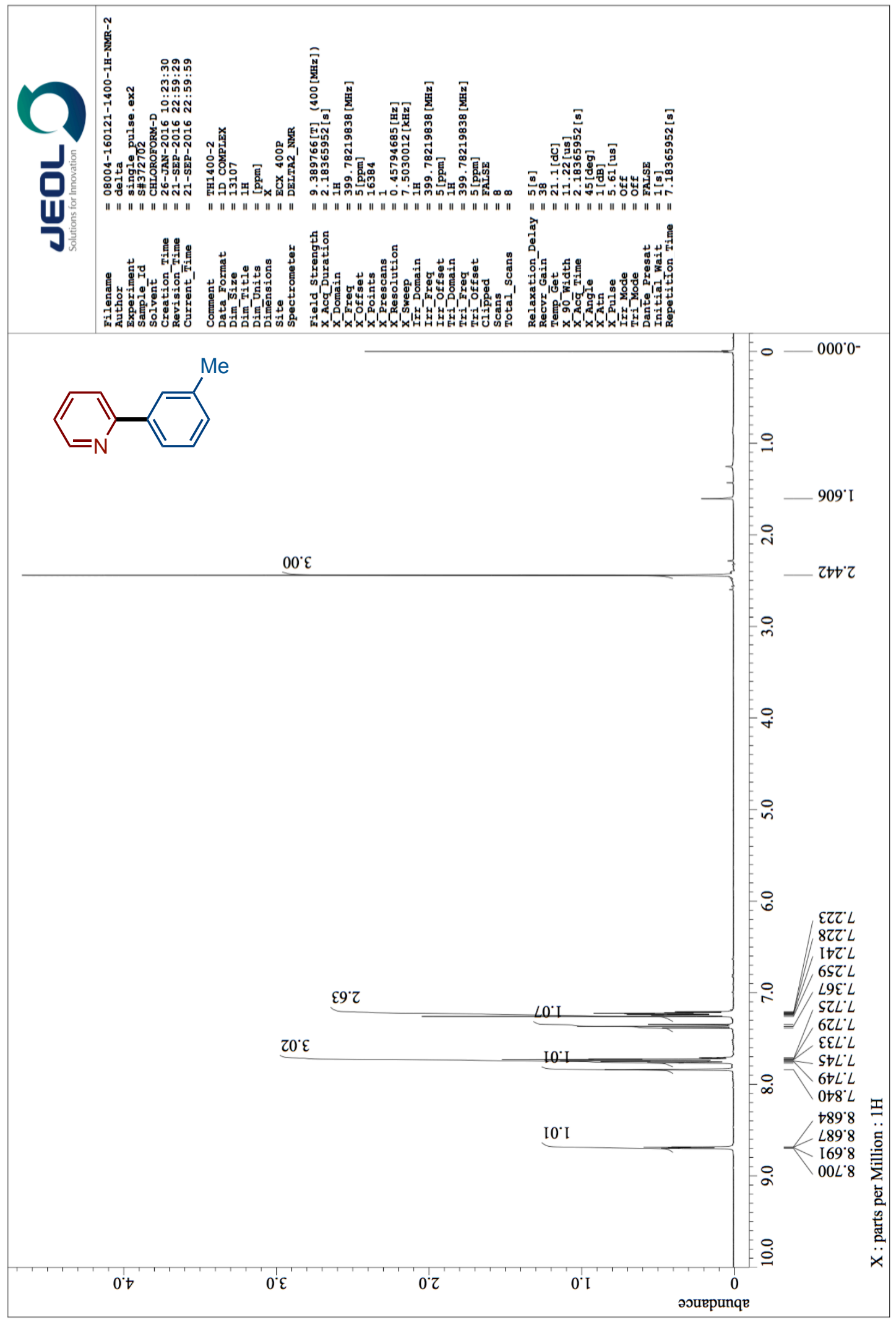


${ }^{13} \mathrm{C}$ NMR 3Ad $\left(100 \mathrm{MHz}, \mathrm{CDCl}_{3}\right)$

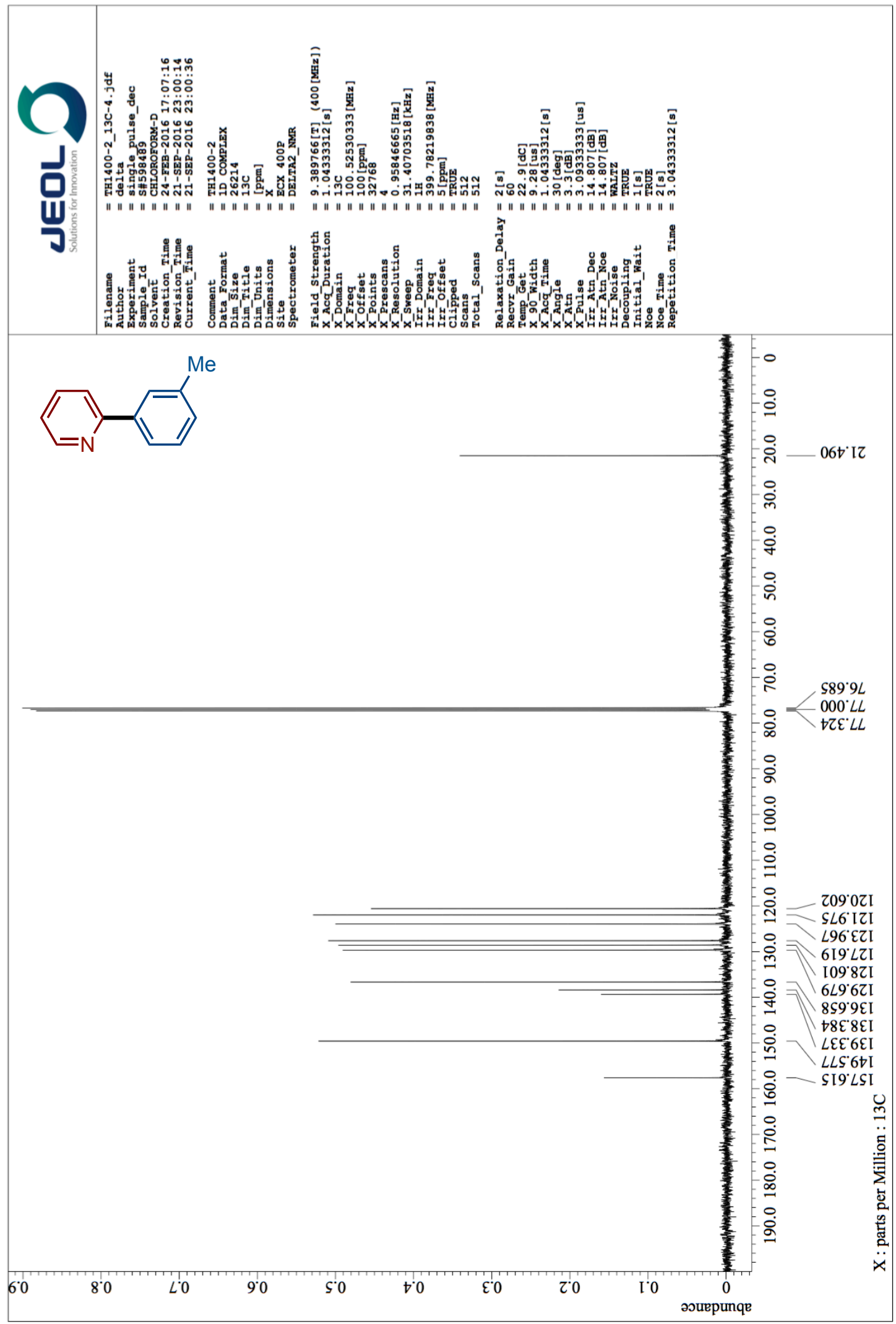


${ }^{1} \mathrm{H}$ NMR 3Ae $\left(400 \mathrm{MHz}, \mathrm{CDCl}_{3}\right)$

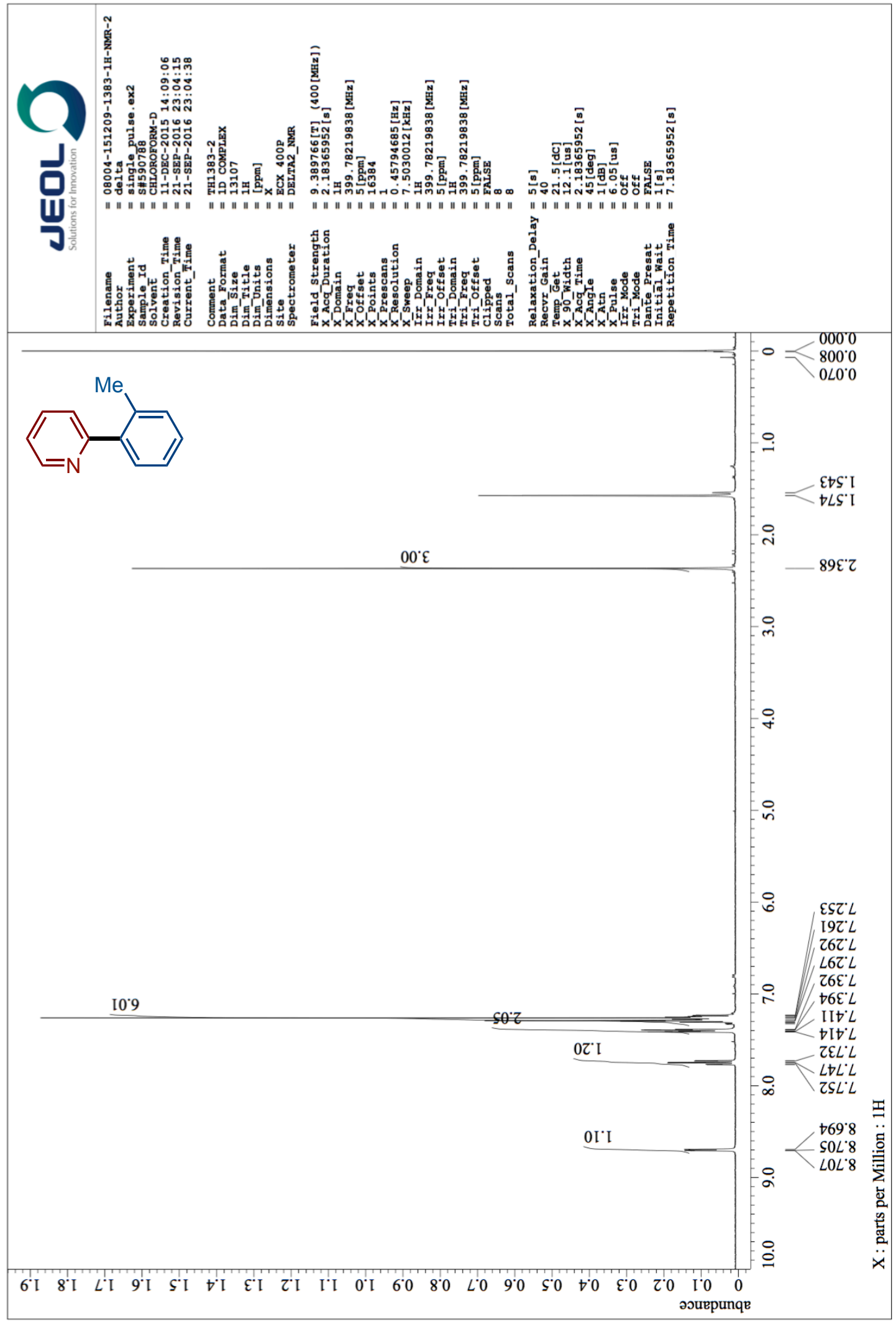


${ }^{13} \mathrm{C}$ NMR 3Ae $\left(100 \mathrm{MHz}, \mathrm{CDCl}_{3}\right)$

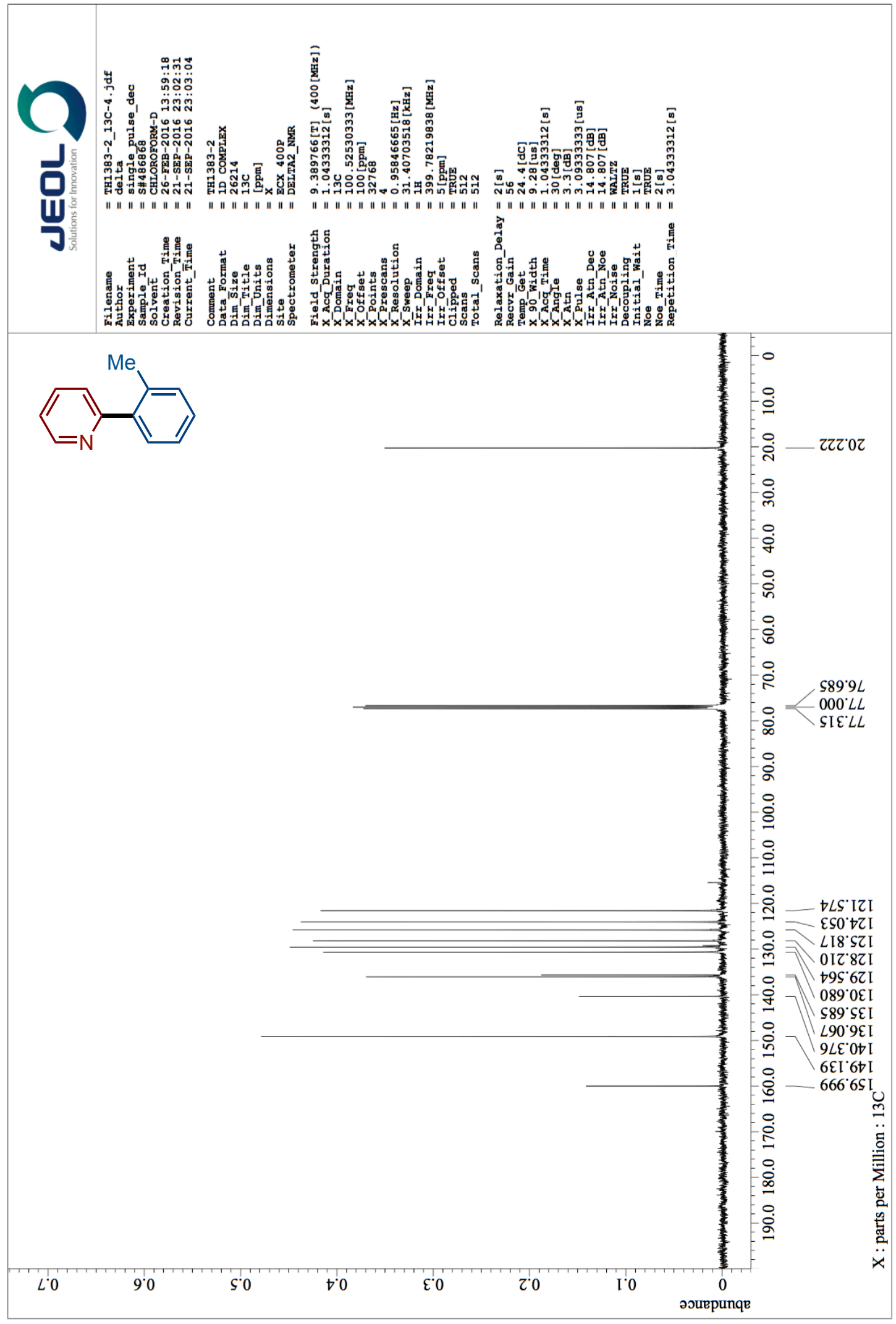


${ }^{1} \mathrm{H}$ NMR 3Af $\left(400 \mathrm{MHz}, \mathrm{CDCl}_{3}\right)$

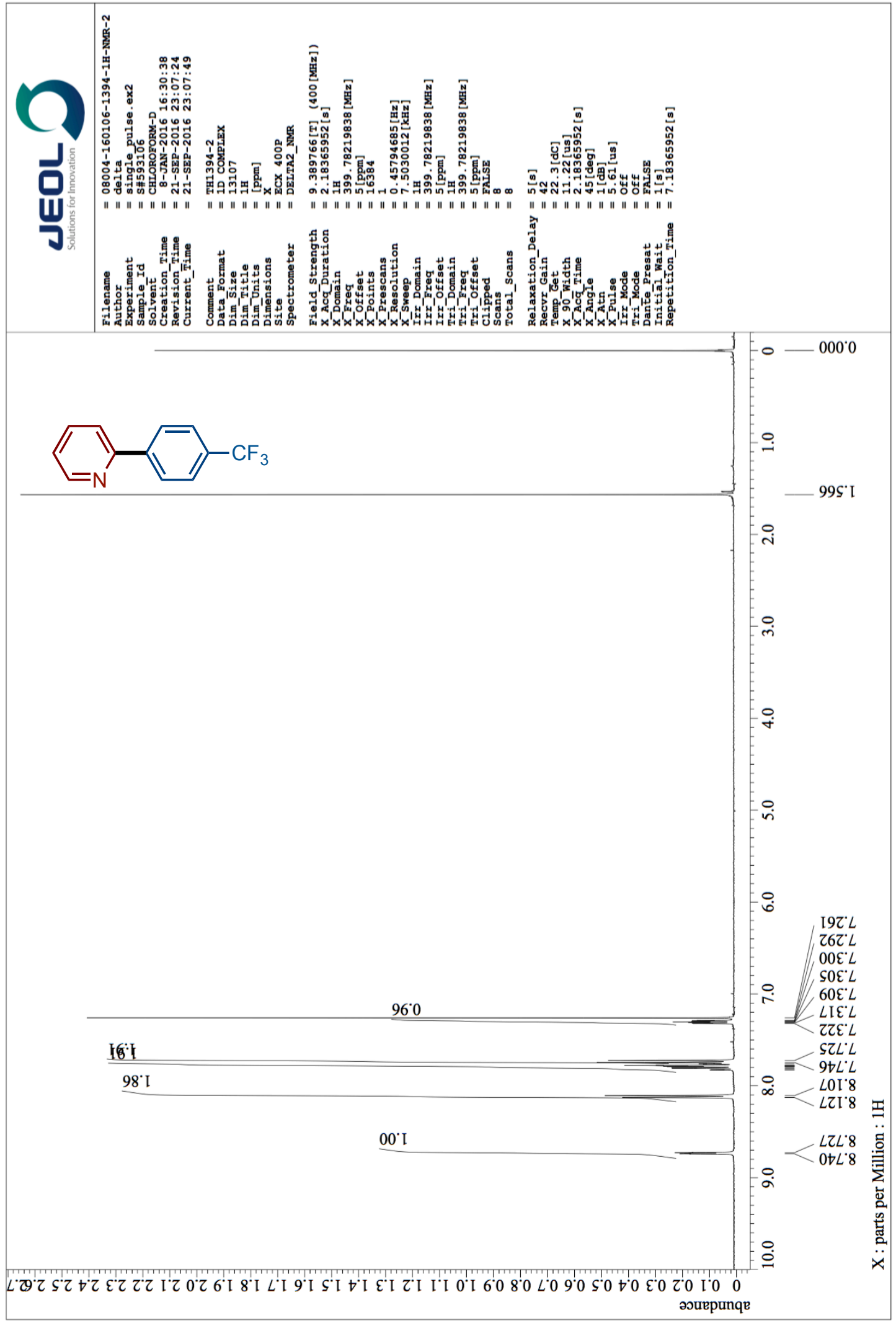


${ }^{13} \mathrm{C}$ NMR 3Af $\left(100 \mathrm{MHz}, \mathrm{CDCl}_{3}\right)$

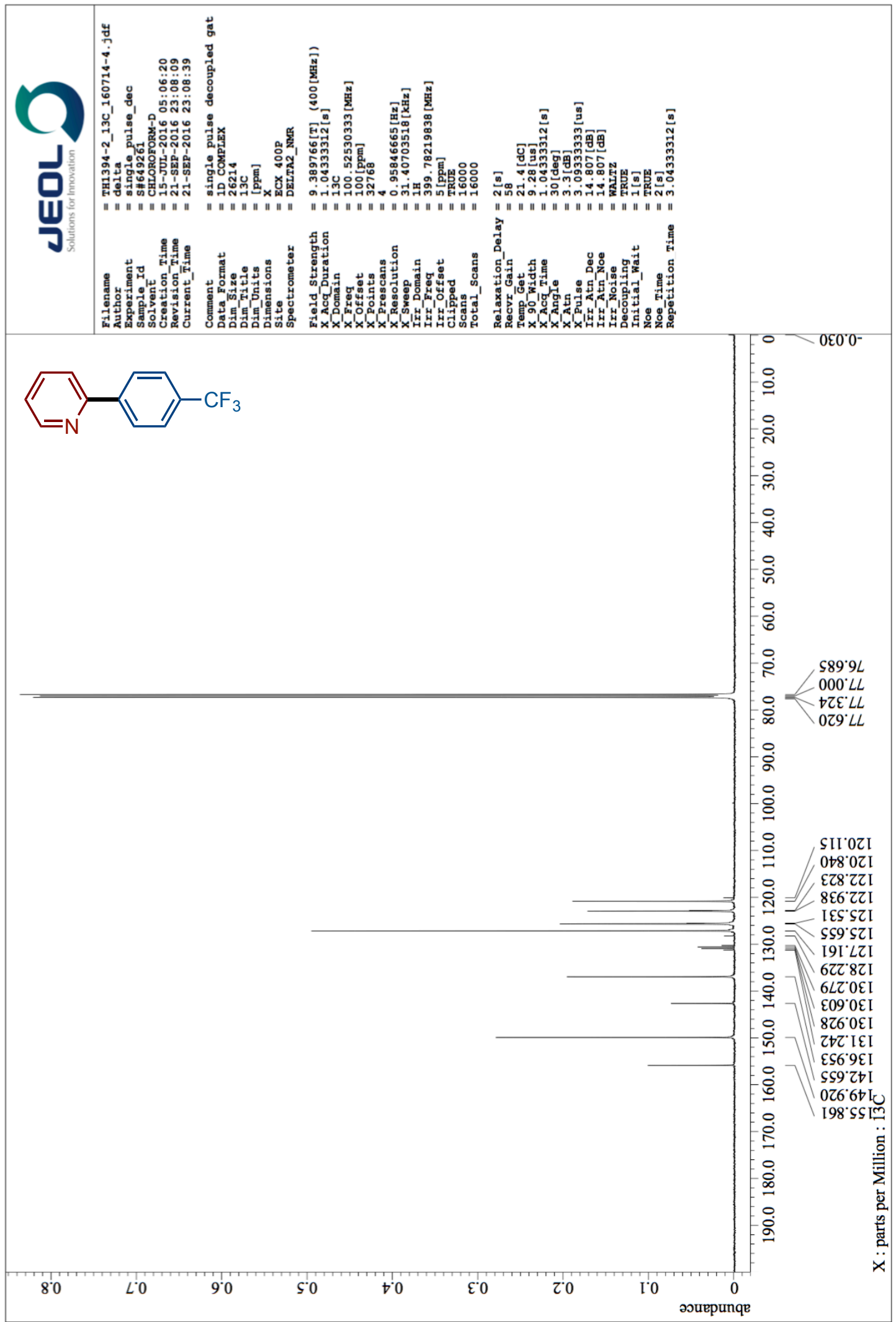


${ }^{1} \mathrm{H}$ NMR 3Ag (400 MHz, $\mathrm{CDCl}_{3}$ )

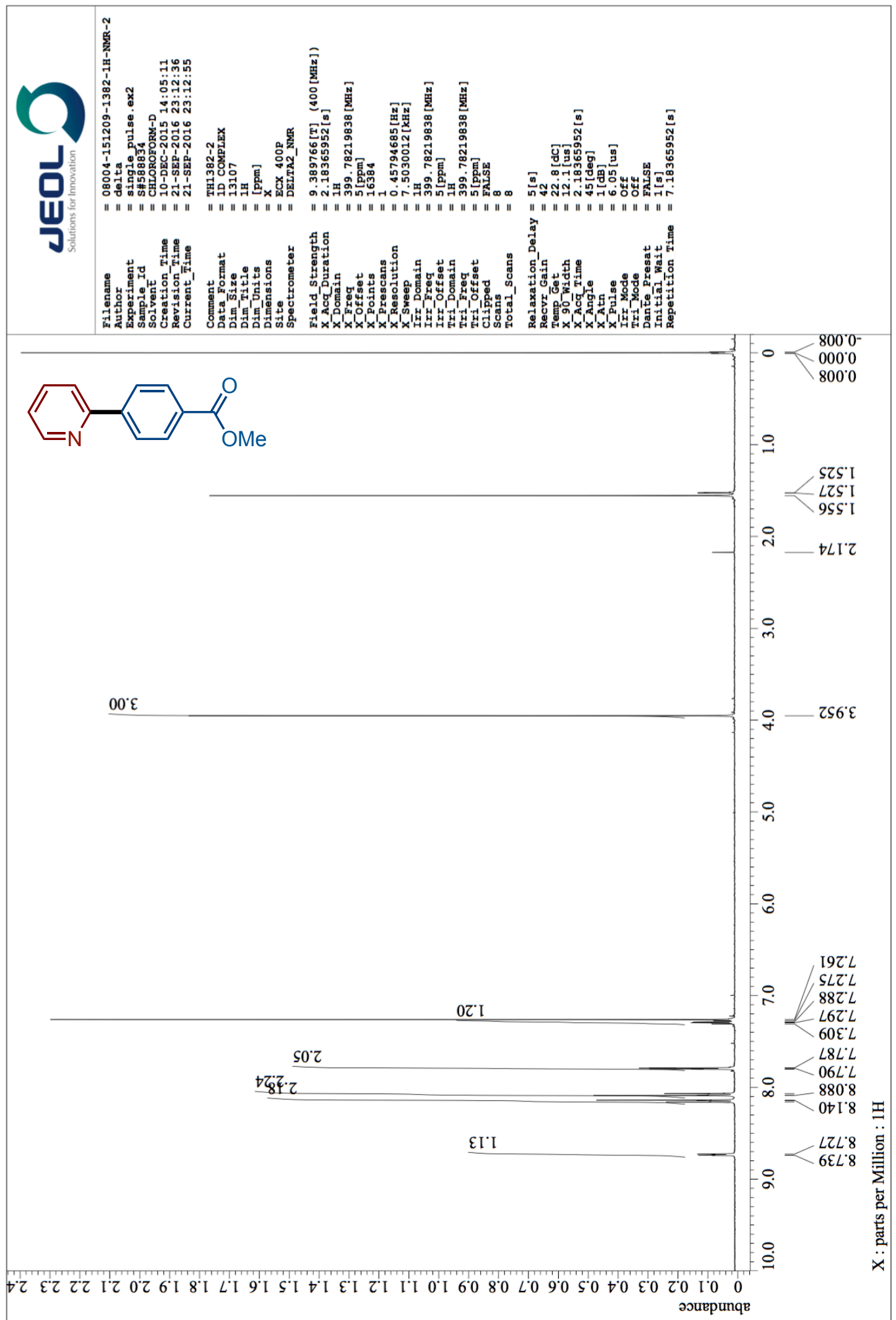


${ }^{13} \mathrm{C}$ NMR 3Ag $\left(100 \mathrm{MHz}, \mathrm{CDCl}_{3}\right)$

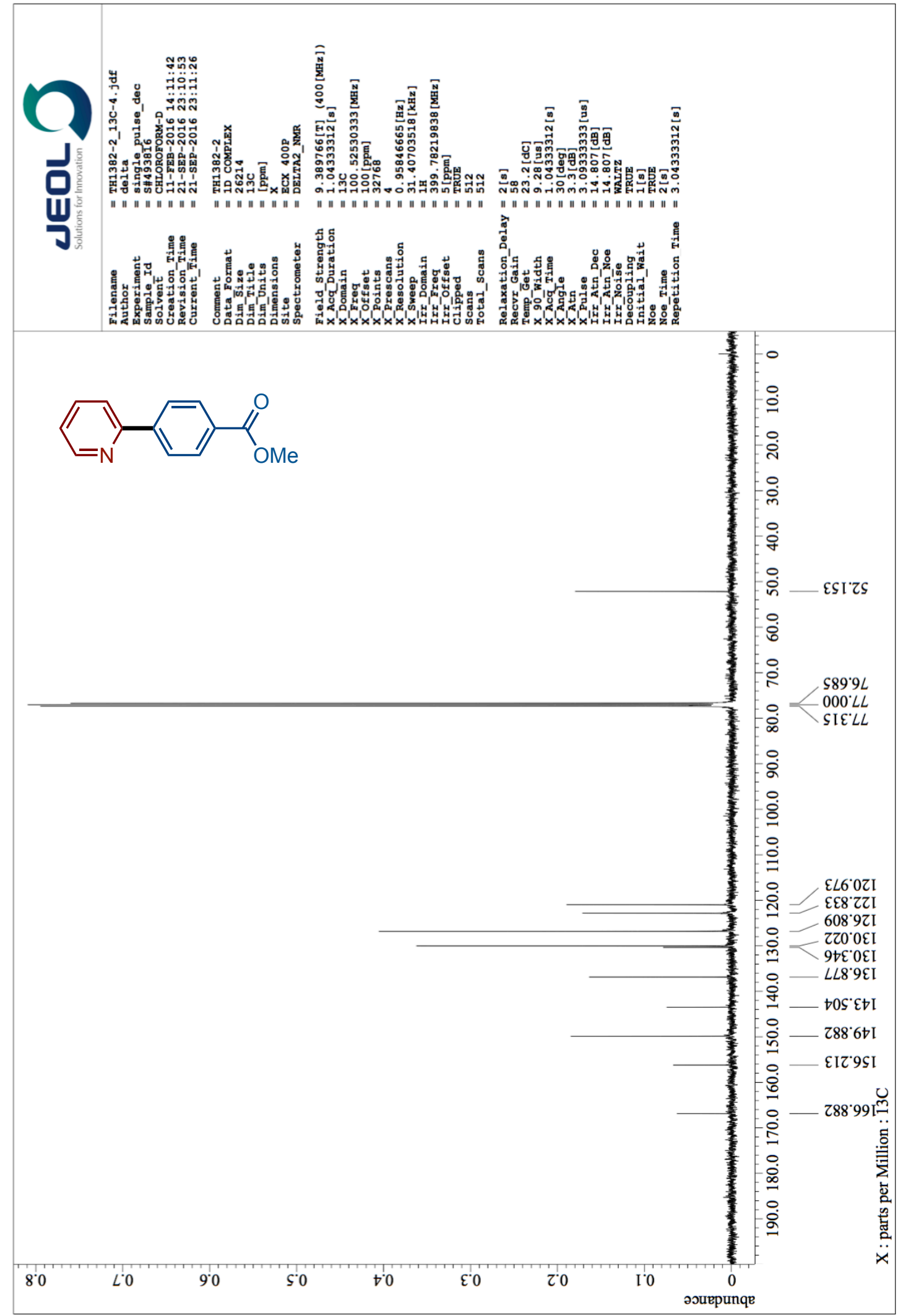


${ }^{1} \mathrm{H}$ NMR 3Ah $\left(400 \mathrm{MHz}, \mathrm{CDCl}_{3}\right)$

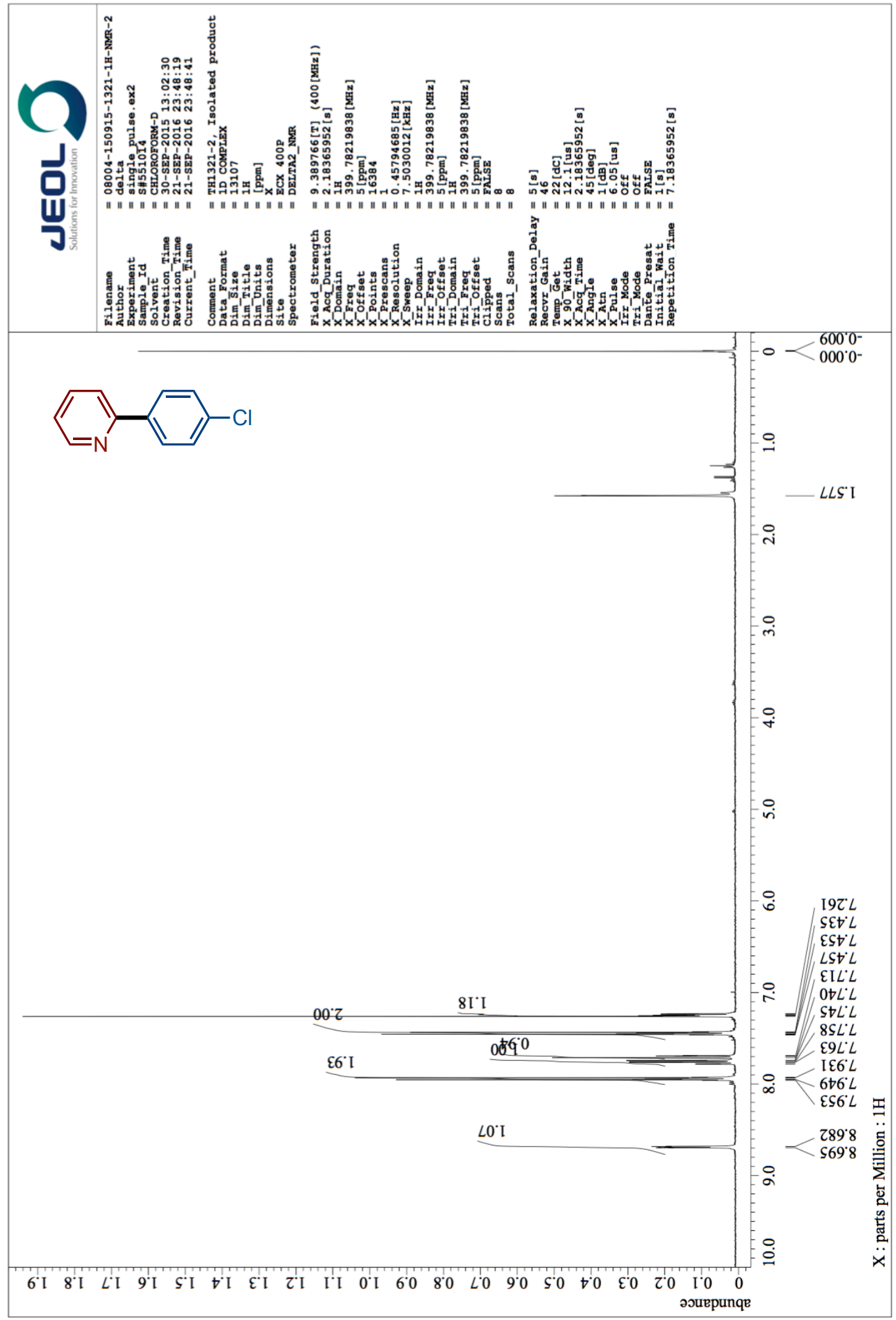


${ }^{13} \mathrm{C}$ NMR 3Ah $\left(100 \mathrm{MHz}, \mathrm{CDCl}_{3}\right)$

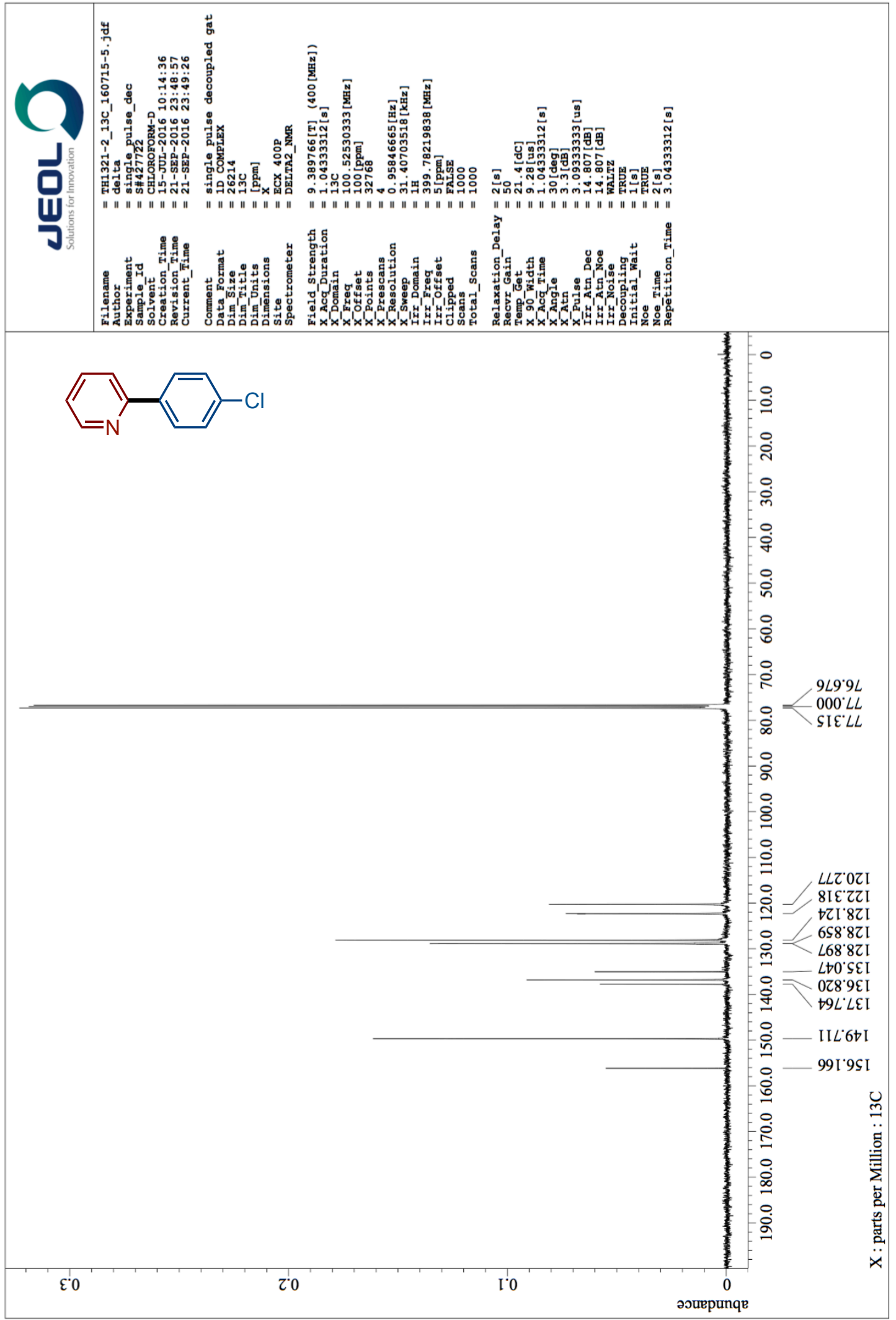


${ }^{1} \mathrm{H}$ NMR 3Ai $\left(400 \mathrm{MHz}, \mathrm{CDCl}_{3}\right)$

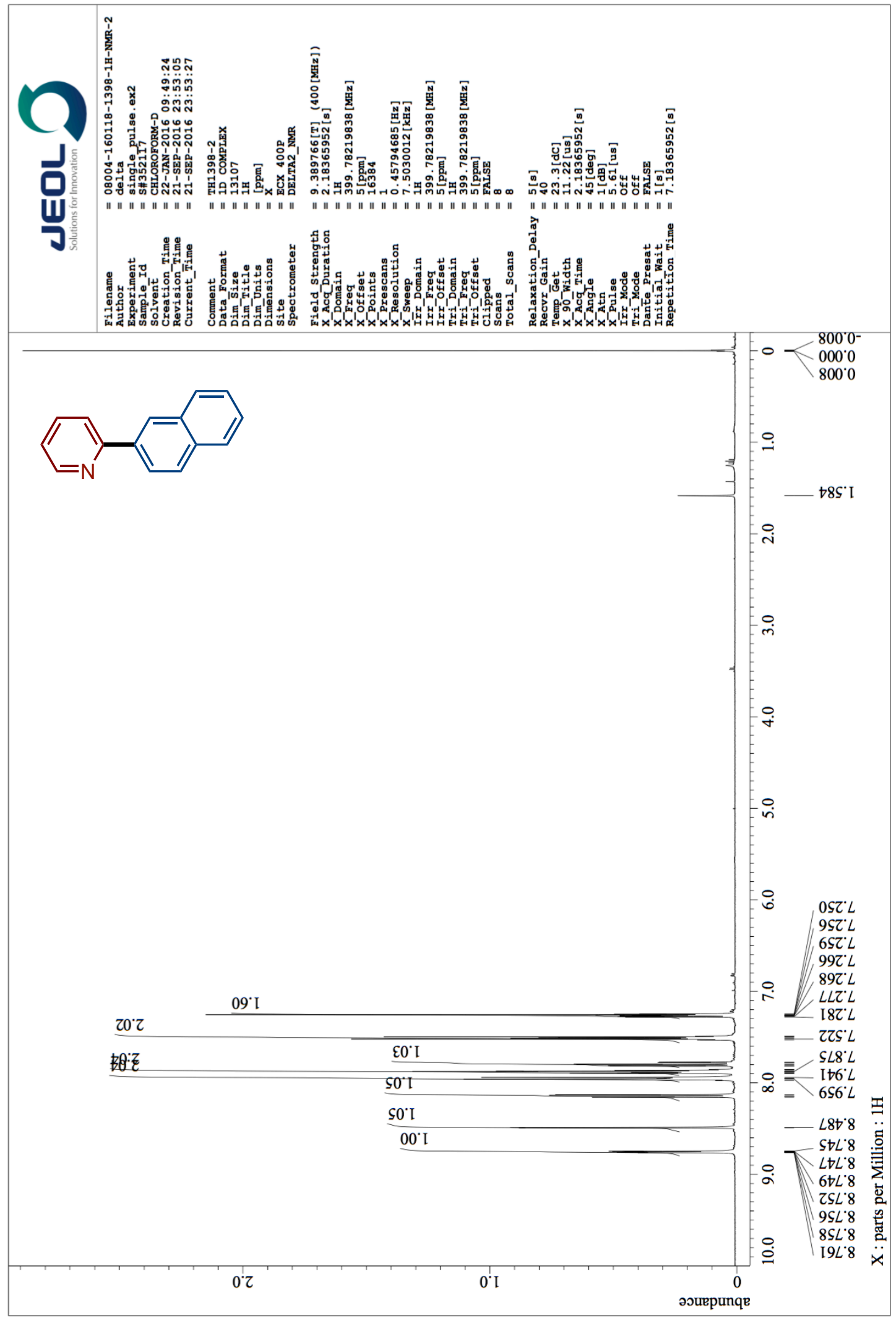


${ }^{13} \mathrm{C}$ NMR 3Ai $\left(100 \mathrm{MHz}, \mathrm{CDCl}_{3}\right)$

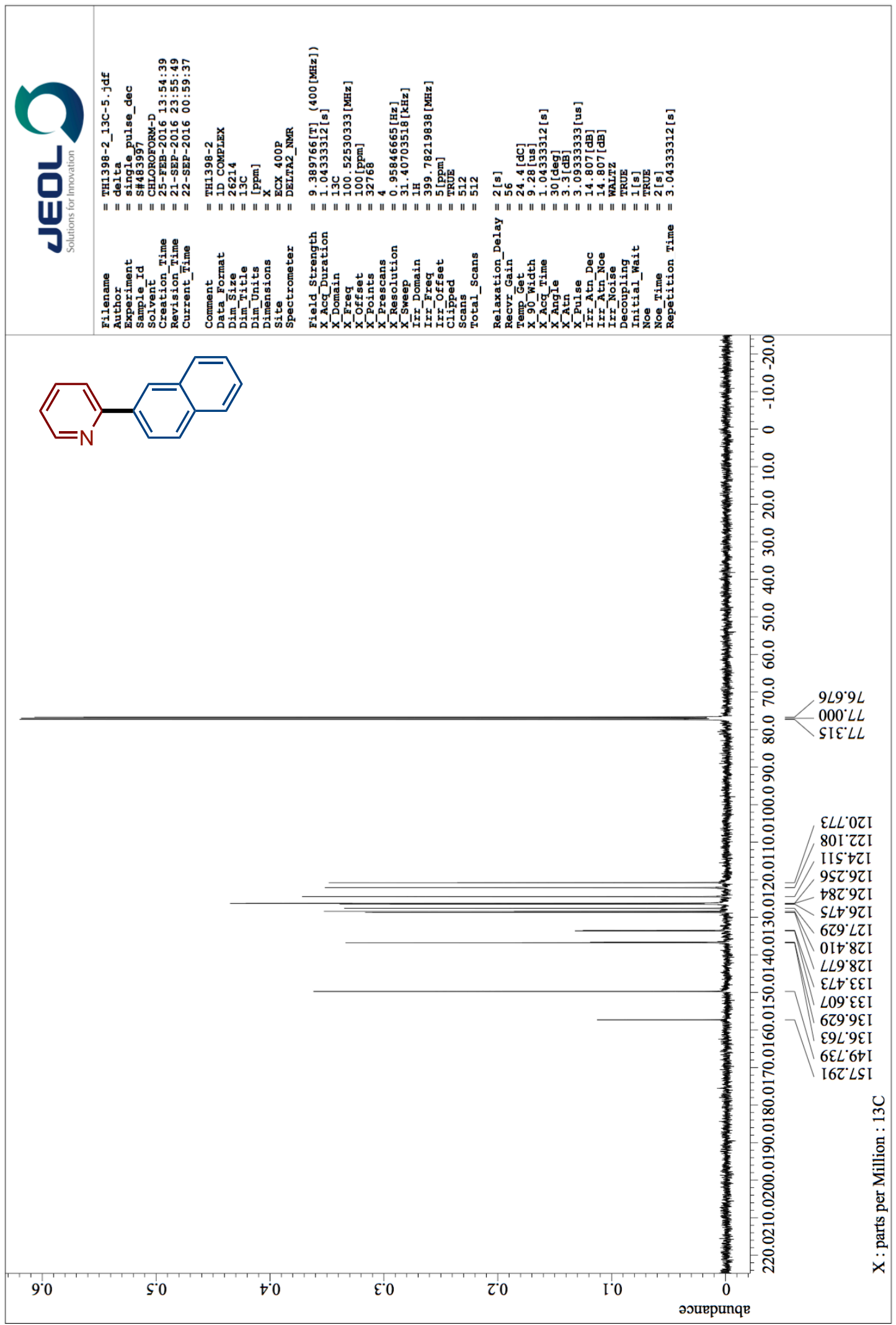


${ }^{1} \mathrm{H}$ NMR 3Aj $\left(400 \mathrm{MHz}, \mathrm{CDCl}_{3}\right)$

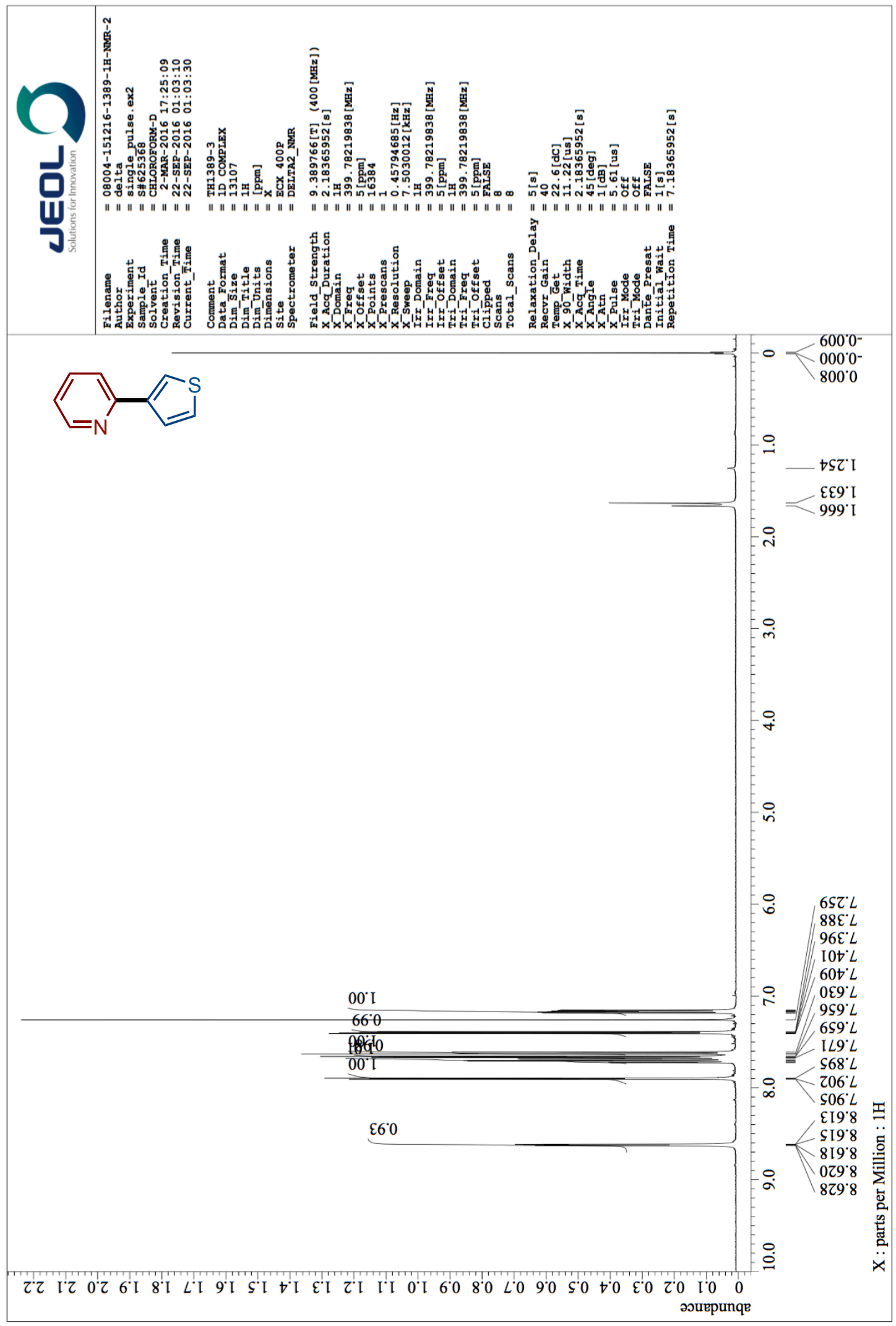


${ }^{13} \mathrm{C}$ NMR 3Aj $\left(100 \mathrm{MHz}, \mathrm{CDCl}_{3}\right)$

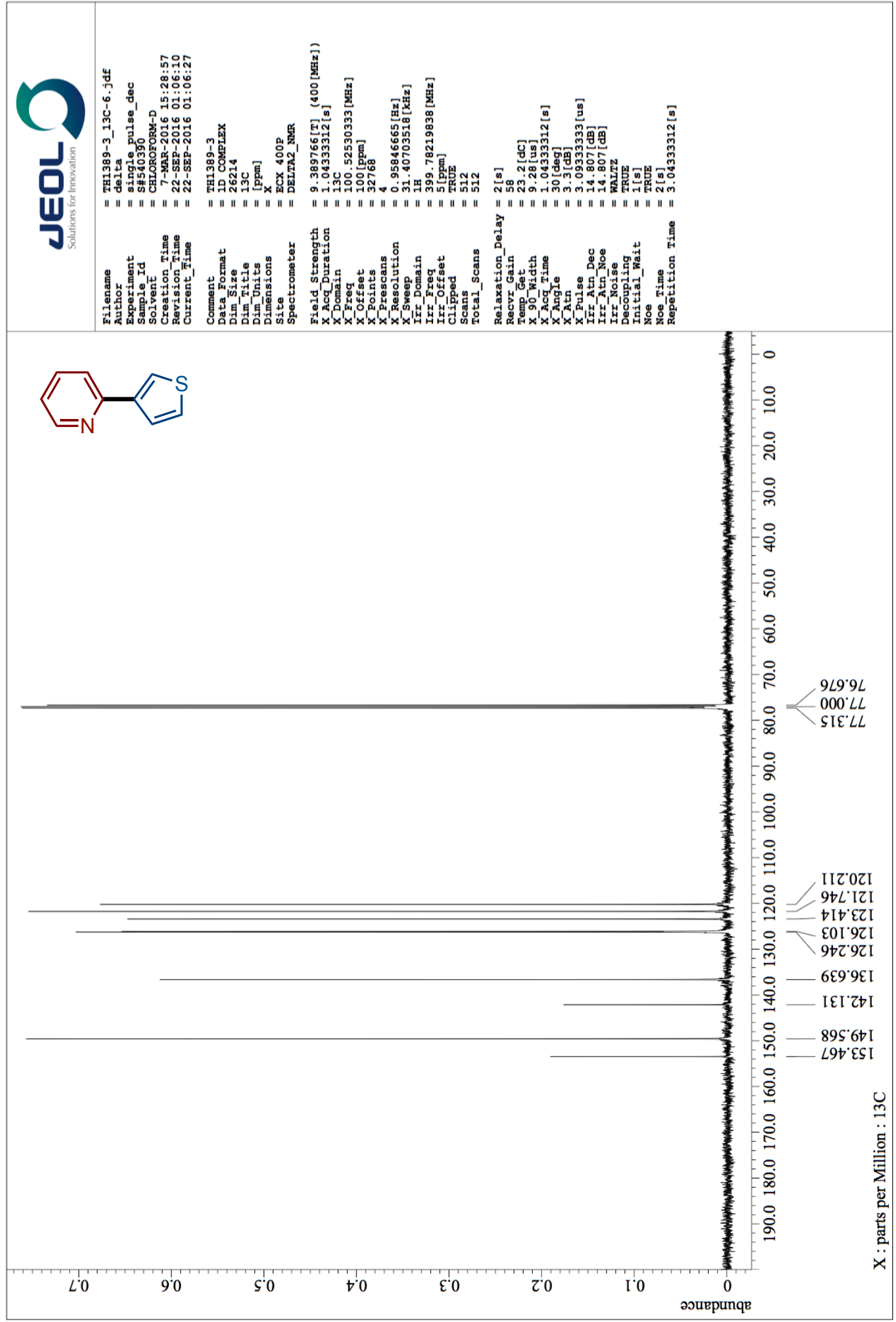


${ }^{1} \mathrm{H}$ NMR 3Ib $\left(400 \mathrm{MHz}, \mathrm{CDCl}_{3}\right)$

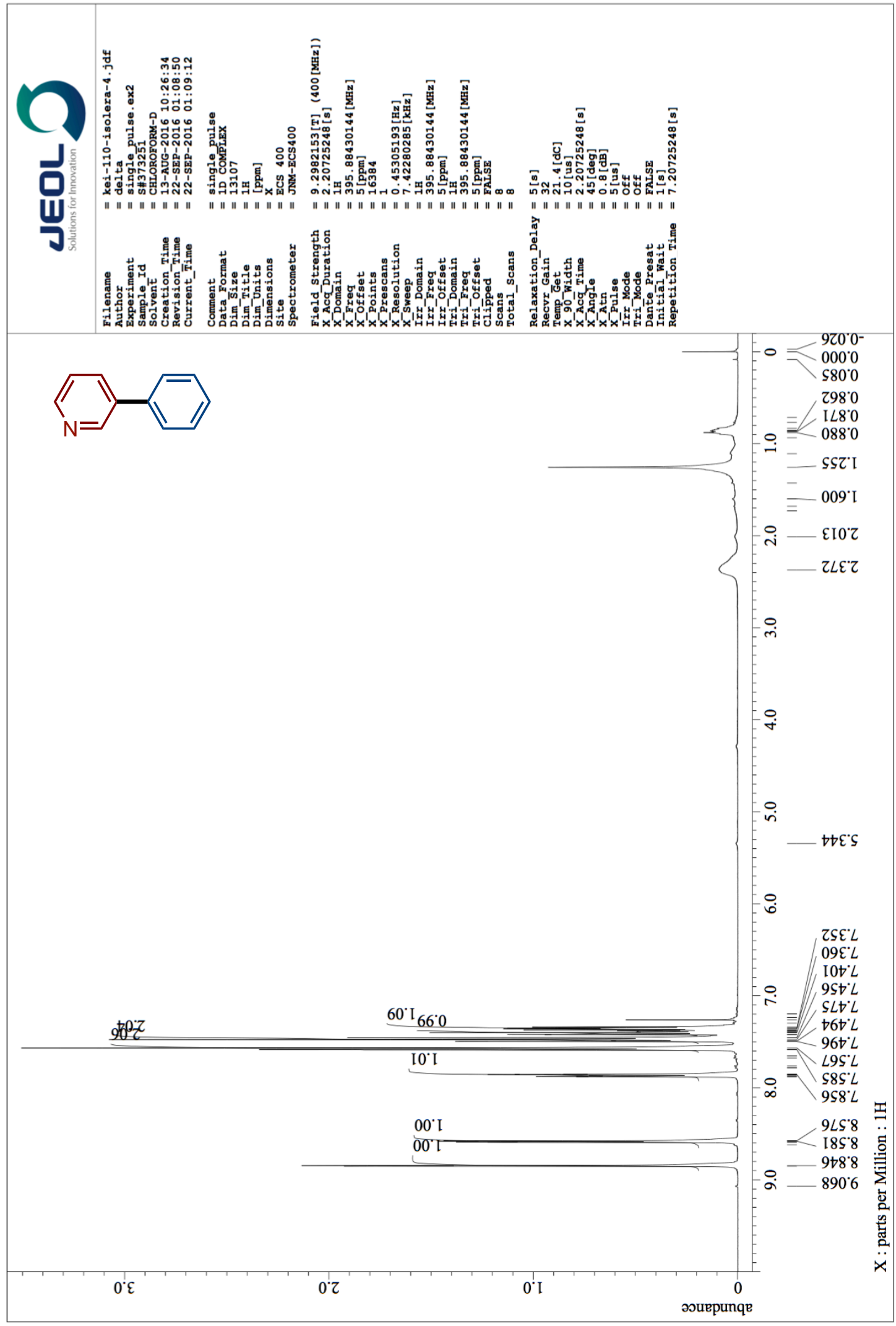


${ }^{13} \mathrm{C}$ NMR 3Ib $\left(100 \mathrm{MHz}, \mathrm{CDCl}_{3}\right)$

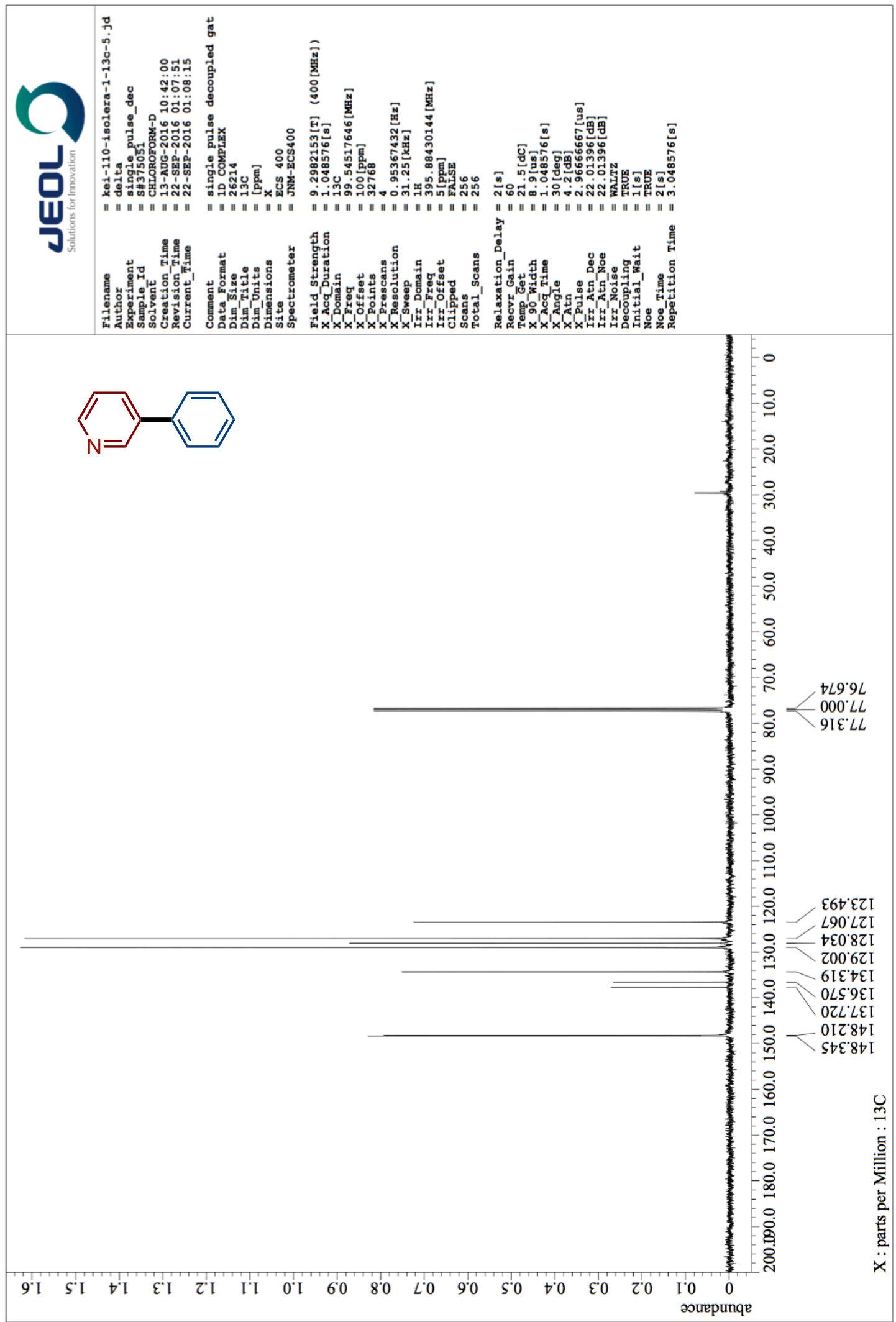


${ }^{1} \mathrm{H}$ NMR 3Ja $\left(400 \mathrm{MHz}, \mathrm{CDCl}_{3}\right)$

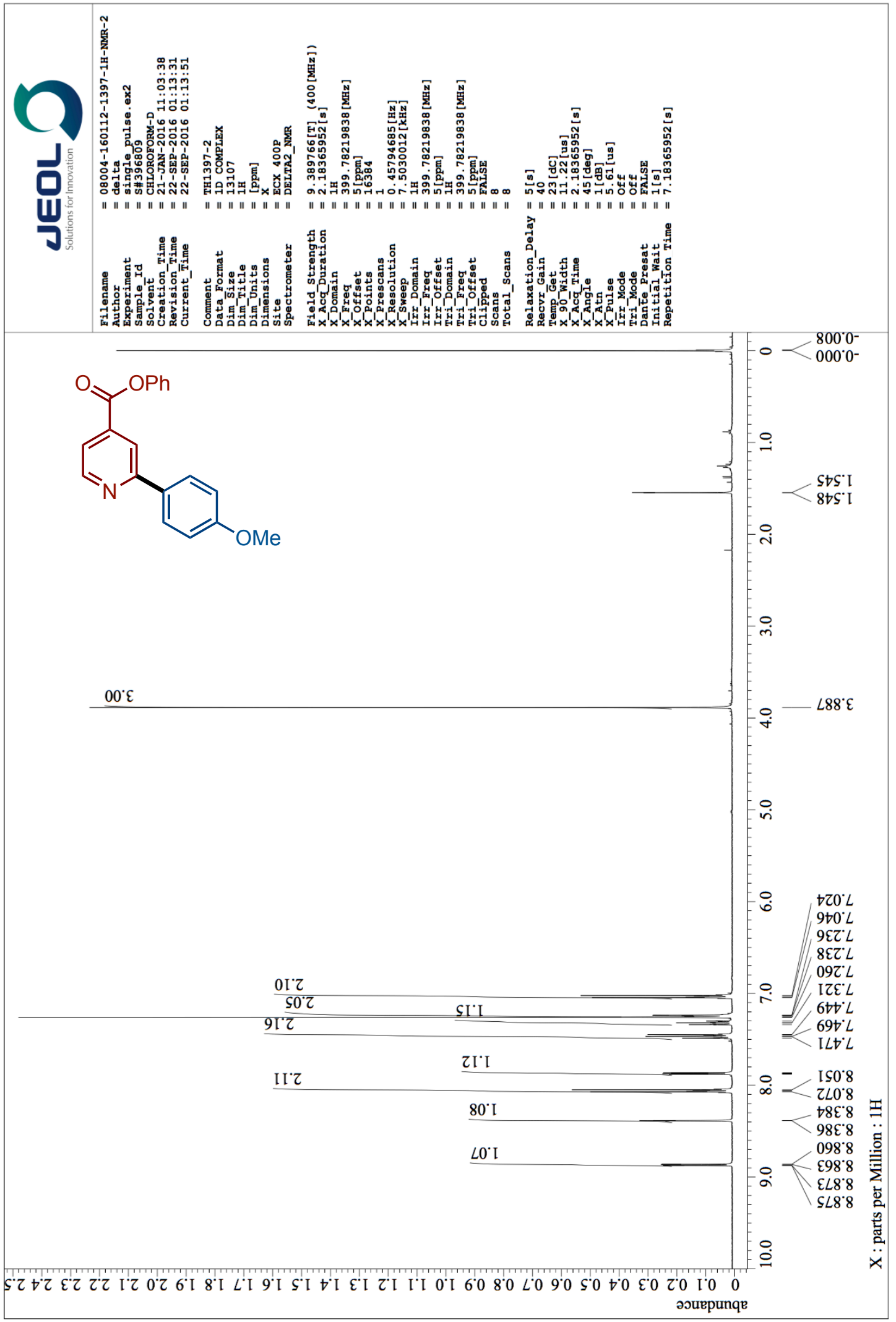


${ }^{13} \mathrm{C}$ NMR 3Ja $\left(100 \mathrm{MHz}, \mathrm{CDCl}_{3}\right)$

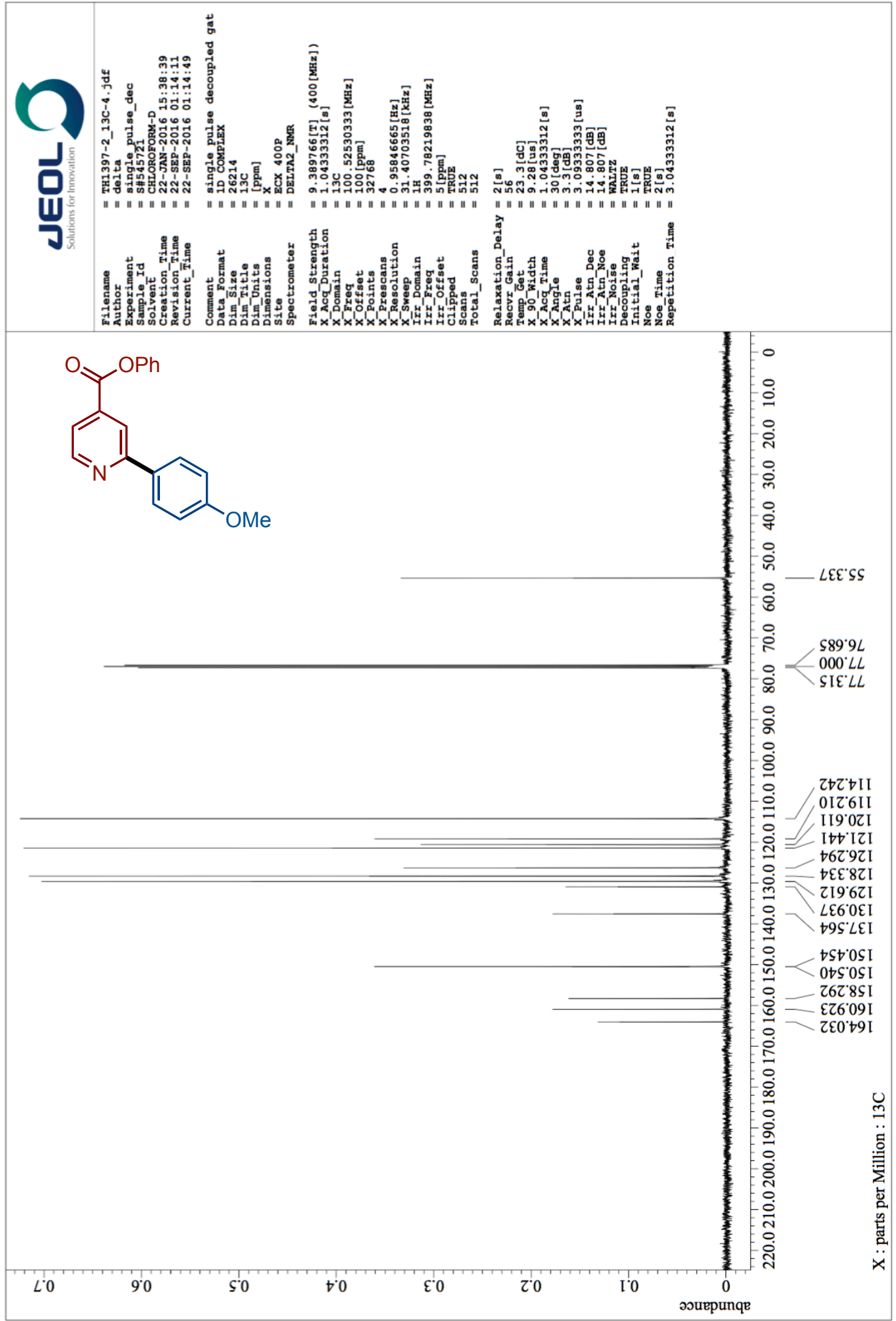


${ }^{1} \mathrm{H}$ NMR $4\left(400 \mathrm{MHz}, \mathrm{CDCl}_{3}\right)$

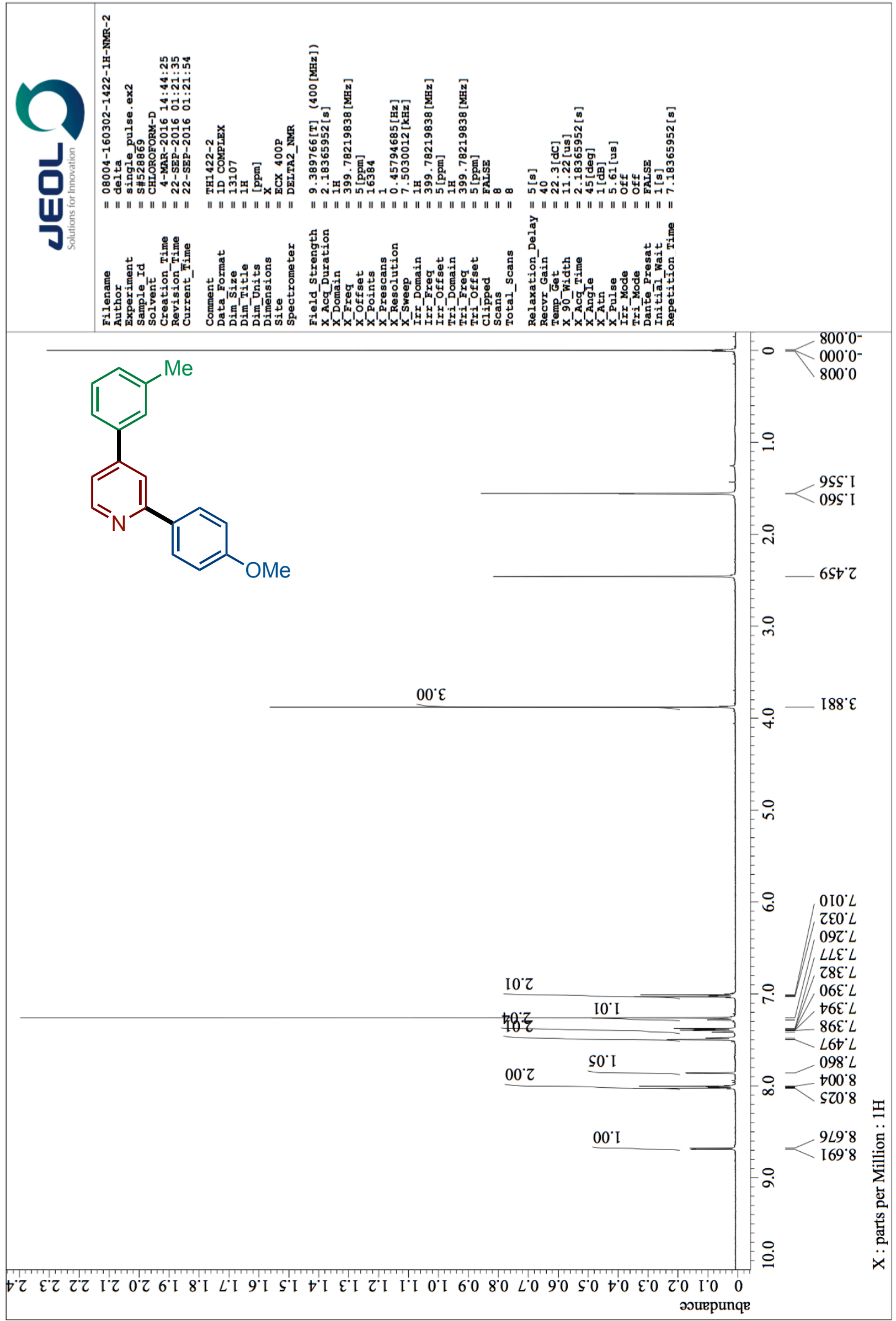


${ }^{13} \mathrm{C}$ NMR $4\left(100 \mathrm{MHz}, \mathrm{CDCl}_{3}\right)$

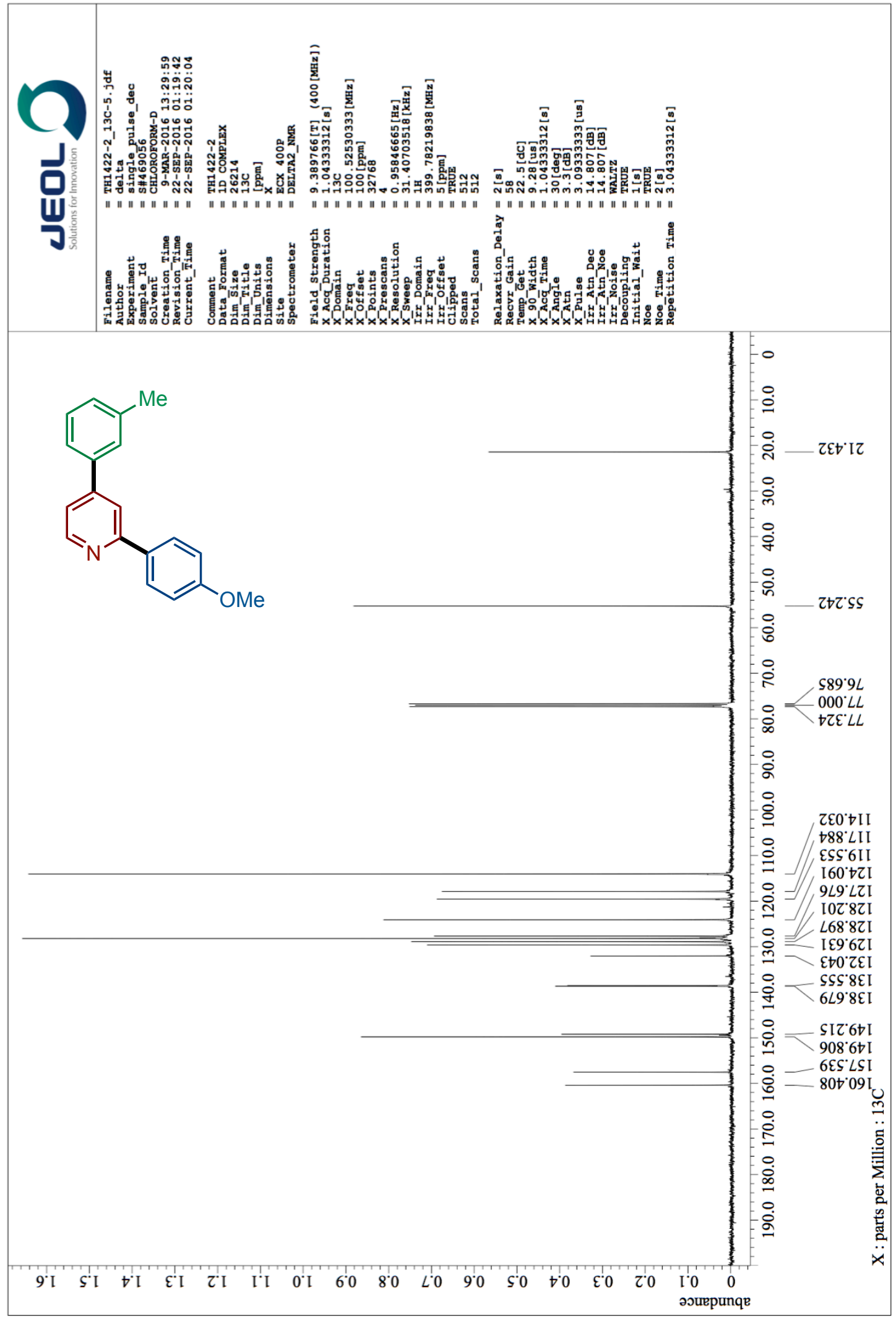

